\title{
FLOWS NEAR COMPACT INVARIANT SETS
}

\author{
Part I \\ PEDRO TEIXEIRA \\ 10 February 2012
}

\begin{abstract}
In this paper it is proved that near a compact, invariant, proper subset of a $C^{0}$ flow on a locally compact, connected metric space, at least one, out of twenty eight relevant dynamical phenomena, will necessarily occur. This result (Theorem 1) shows that assuming the connectedness of the phase space, implies the existence of a considerably deeper classification of topological flow behaviour in the vicinity of compact invariant sets than that described in the classical theorems of Ura-Kimura and Bhatia. The proposed classification brings to light, in a systematic way, the possibility of occurrence of orbits of infinite height arbitrarily near the compact invariant in question, and this under relatively simple conditions. Singularities of $C^{\infty}$ vector fields displaying this strange phenomenon occur in every dimension $n \geq 3$ (in this paper, a $C^{\infty}$ flow on $\mathbb{S}^{3}$ exhibiting such an equilibrium is constructed). Near periodic orbits, the same phenomenon is observable already in dimension 4 (and on every manifold of dimension $n \geq 5$ ). As a corollary to the main result, an elegant characterization of the topological Hausdorff structure of the set of all compact minimal sets of the flow is obtained (Theorem 2).
\end{abstract}

MSC 2010: primary 37B25, 37B99; secondary 37C27, 37C70, 58K45.

keywords: topological behaviour of $C^{0}$ flows, compact invariant sets, compact minimal sets, topological Hausdorff structure, non-hyperbolic singularities and periodic orbits, orbits of infinite height.

In memoriam Vladimir I. Arnol'd
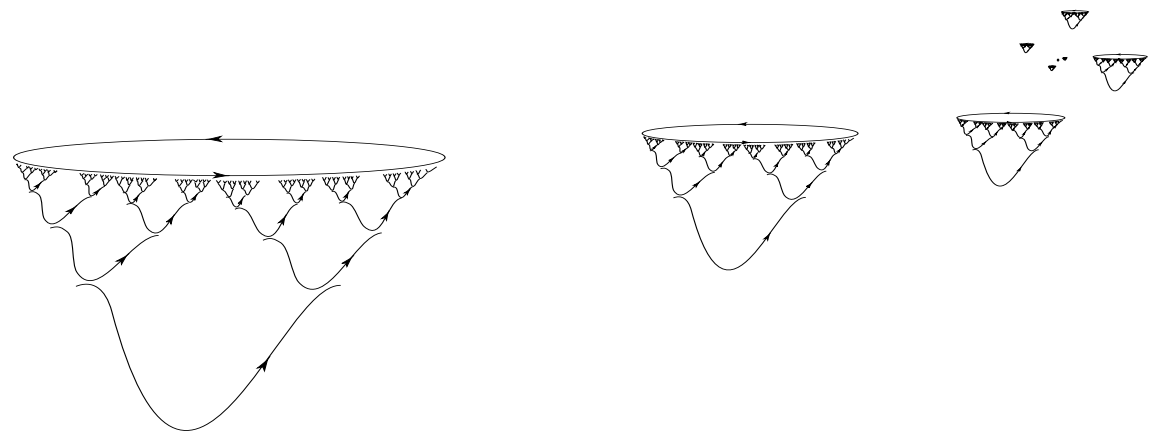

Fig. 1: Example of the occurrence of orbits of infinite height. This figure illustrates case 8.6 of Theorem 1. 
CONTENTS.

1. Introduction.

2. Definitions and basic results.

3. Presentation of the main result. Corollaries.

4. Special orbital structures.

5. Lemmas.

6. The main theorem. Topological structure of $\mathrm{CMin}(M)$.

7. Independent realizations. Examples.

8. Proof of lemma 7. Two topological lemmas.

\section{INTRODUCTION.}

The present work establishes a natural classification of topological behaviour of $C^{0}$ flows near arbitrary compact invariant sets $K$, on locally compact connected metric spaces $M$ (e.g. on 2nd countable, Hausdorff, compact or not, connected manifolds). It can be seen as a considerably deep generalization of a classical topological-dynamical result of Ura and Kimura [13] and Bhatia (see e.g. [1], p. 114), when the hypothesis of connectedness of the phase space is added. The Ura-Kimura-Bhatia Theorem states that if $M$ is a locally compact (but not necessarily connected) metric space and $K$ is as above, then at least one of the following four cases takes place:

I. $K$ is an attractor (i.e. asymptotically stable)

II. $K$ is a repeller (i.e. negatively asymptotically stable)

III. there are points $x, y \in M \backslash K$ such that $\emptyset \neq \alpha(x) \subset K$ and $\emptyset \neq \omega(y) \subset K$

IV. every neighbourhood of $K$ contains a compact invariant set that contains $K$ as a proper subset (i.e. $K$ is not an isolated invariant set).

While valid for very general flows/phase spaces and despite its importance, namely, in persistence theory (see $e . g$. the preface of [11]), the above result has, in our opinion, an obvious serious limitation that hinders the possibility of a natural, substantial deepening of the classification it proposes: since the phase space is not assumed to be connected, a (nonvoid) compact invariant set $Q \varsubsetneqq M$ may be open in $M$. This makes $Q$ simultaneously an attractor and a repeller, while in fact $Q$ neither attracts nor repels a single point outside itself. Actually as $M \backslash Q$ is closed, sufficiently near but outside $Q$ the flow is vacuous!

Adding the assumption of connectedness of the phase space dramatically improves the possibility of partially describing the "dynamical landscape" in the vicinity of a compact invariant set. Natural considerations lead to the identification of twenty five possible relevant dynamical phenomena that fall under case IV of the Ura-Kimura-Bhatia Theorem. Moreover the whole twenty eight cases 
are distributed among five groups, two cases belonging to distinct groups being incompatible i.e cannot be simultaneously satisfied. A key role in the classification is played by compact invariant sets $\emptyset \neq K \subsetneq M$ that are either attractors or repellers or isolated from minimal sets and stagnant. By the later we mean that for some neighbourhood $U$ of $K, U \backslash K$ contains no minimal set of the flow and in addition condition III above is satisfied. Although the main result of this paper (Theorem 1) goes much deeper, a "flavour" of some of its most important conclusions is given in the following corollary:

Let $M$ be a locally compact, connected metric space with a $C^{0}$ flow $\theta$ and $K$ a compact, invariant, proper subset of $M$. Then:

either I) $K$ is an attractor

or II) $K$ is a repeller

or III) $K$ is isolated from minimal sets and stagnant.

or IV) there is a nonvoid, compact, connected invariant set $Q \subset \mathrm{bd} K$ and a sequence $\Lambda_{n} \subset M \backslash K$ of compact minimal sets of the flow such that the following three conditions hold:

- $\left(\Lambda_{n}\right)$ converges to $Q$ in the Hausdorff metric

- all $\Lambda_{n}^{\prime}$ s belong to the same one of the following three classes: equilibrium orbits, periodic orbits, aperiodic compact minimals sets

- either all $\Lambda_{n}^{\prime} s$ are attractors or they are repellers or they are all isolated from minimal sets and stagnant.

or $\mathbf{V )}$ for all sufficiently small open neighbourhood $U$ of $K$, the compact minimals sets contained in $U \backslash K$ form a nonvoid $\mathfrak{c}$-dense in itself set i.e. any neighbourhood of a compact minimal set $\Lambda \subset U \backslash K$ contains a continuum of compact minimal sets. 1

Finally if none of conditions $\mathrm{I}$ to $\mathrm{V}$ holds, then orbits of infinite height ${ }^{2}$ will necessarily occur arbitrarily near but outside $K$, more precisely,

VI) Given any neighbourhood $U$ of $K$, there is a sequence of orbits $\gamma_{n} \subset U \backslash K$ such that

$$
\operatorname{cl} \gamma_{1} \supsetneq \operatorname{cl} \gamma_{2} \supsetneq \cdots \cdots \supsetneq \operatorname{cl} \gamma_{n} \supsetneq \cdots \cdots
$$

\footnotetext{
${ }^{1}$ note that $M$ being locally compact, every sufficiently small neighbourhood of $K$ may contain only compact minimal sets, hence in both IV and $\mathrm{V}$ we may actually replace compact minimal set(s) by minimal set(s) everywhere.

${ }^{2}$ An orbit $\gamma_{0}$ is of infinite height if there is an infinite strict inclusion chain of orbit closures $\operatorname{cl} \gamma_{0} \supsetneq \operatorname{cl} \gamma_{1} \supsetneq \cdots \cdots \supsetneq \operatorname{cl} \gamma_{n} \supsetneq \cdots \cdots$

(some authors call $\gamma_{0}$ an orbit of infinite depth).
} 

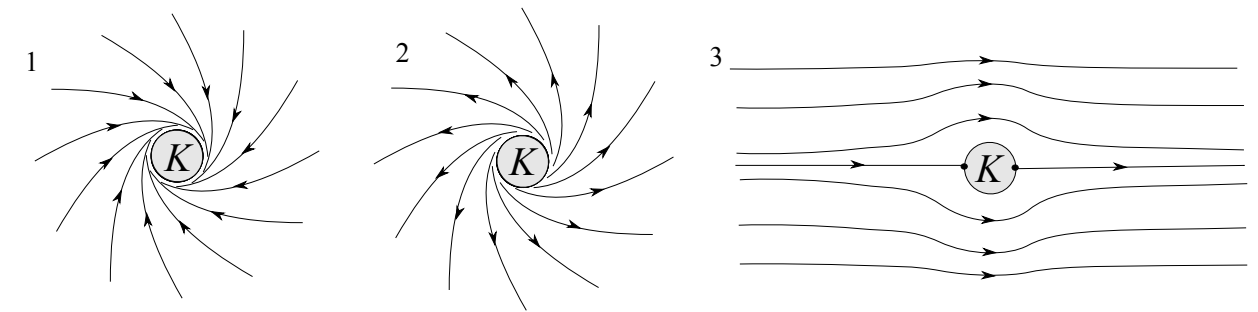

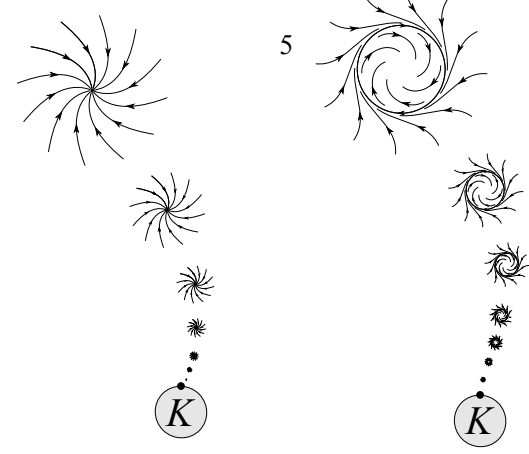

6

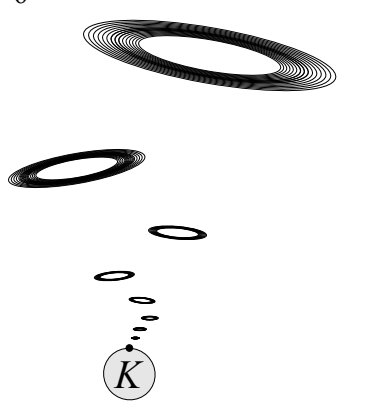

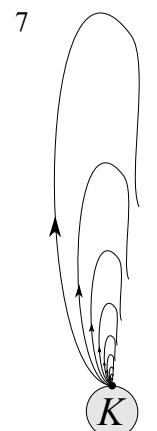

Fig. 2: Examples: 1 - case I; 2 - case II; 3 - case III; $4 \& 5$ - case IV; 6 - case V; 7 - case VI.

Therefore, if $K$ is neither an attractor nor a repeller and conditions III and IV fail, then a "super-abundance" of compact minimal sets (case V) or an outstanding kind of limit behaviour (case VI) will emerge arbitrarily near (but outside) $K$. The possible occurrence of the later disturbing dynamical phenomenon is not a mere theoretical speculation: in section 7 a $C^{\infty}$ flow on $\mathbb{S}^{3}$ exhibiting it is given. This is made possible by the existence of a $C^{\infty}$ flow without minimal sets on a non-compact surface of infinite genus $\left(C^{\infty}-\right)$ embeddable in $\mathbb{R}^{3}$ (see Beniere and Meigniez [2] and the pioneer work of Inaba [6]). In a subsequent paper [12] we shall actually show that our classification is both pertinent and non-redundant: each of the twenty eight cases it describes admits an independent realization either by a $C^{\infty}$ flow on a (2nd countable, Hausdorff) compact, connected manifold or by a $\left(C^{0}\right)$ subflow corresponding to a compact, connected invariant subset of such $C^{\infty}$ flow (see section 7).

Among its many interesting consequences, the classification theorem ${ }^{3}$ has remarkable, and somewhat unexpected, implications in the topological structure of the set $\mathrm{CMin}(M)$ of all compact minimal sets of the flow, endowed with the Hausdorff metric $d_{H}$ (see sections 3 and 6). As an example, let $\mathfrak{A}$ be the set of compact minimal sets that are either attractors or repellers or isolated from minimal sets and stagnant. Then,

\footnotetext{
${ }^{3}$ see section 3 for a first illustrated presentation and section 6 for the full statement.
} 
"If a $C^{0}$ flow on a locally compact, connected metric space $M$ has only countably many compact minimal sets and displays no orbits of infinite height then $\mathfrak{A}$ is necessarily open dense in $\mathrm{CMin}(M)$ (in the Hausdorff metric)".

If the flow displays uncountably many compact minimal sets, then a preliminary result (lemma 7) permits to establish a topological decomposition of $\mathrm{CMin}(M)$, in a certain sense analogue to that of Cantor-Bendixson Theorem for Polish spaces:

"If $\operatorname{CMin}(M)$ is uncountable then all but a countable number of compact minimal sets of the flow have a continuum of compact minimal sets on each of their neighbourhoods".

This result brings to light the corresponding analogue proposition $\Sigma$ for the set $\operatorname{Per}(M)$ of all periodic orbits of the flow:

"If $\operatorname{Per}(M)$ is uncountable then all but a countable number of periodic orbits of the flow have a continuum of periodic orbits on each of their neighbourhoods".

There are no counterexamples to $\Sigma$ within "standard" Dynamical Systems Theory: its negation implies the Negation of the Continuum Hypothesis (this is actually a purely topological fact, depending only on the Hausdorff metric separability of $\operatorname{Per}(M))$. Hence $\Sigma$ is either demonstrable in ZermeloFraenkel+Axiom of Choice (ZFC) or it is independent of this standard axiomatic (see section 6).

Despite its topological nature, the greatest interest of Theorem 1 lies in the context of $C^{r \geq 0}$ flows on $C^{r}$ manifolds. 4 Particularly noteworthy is, perhaps, its contribution to the understanding of what can happen, from the dynamical point of view, in two potential "nightmare" phenomena of differentiable dynamics: non-hyperbolic singularities and periodic orbits.5 To see how hopeless standard "analytic" methods may be in the study of the former, even in low dimensions, consider, for example, the case of complete $C^{\infty}$ vector fields on $\mathbb{R}^{2}$ having the origin $O$ as an isolated $f l a t^{6}$ singularity. It is not difficult to see that there is a continuum of such vector fields $X_{i}, i \in \mathbb{R}$, that are mutually topologically non equivalent ${ }^{7}$ at $O$, and whose local topological behaviour at

\footnotetext{
${ }^{4}$ except if otherwise mentioned, manifolds are always assumed to be 2 nd countable, Hausdorff, compact or not, connected and boundaryless.

5 in relation to the later, the seven cases 7.1 to 7.6 and 10.1 of Theorem 1 (section 6) are apriori ruled out (since singularities cannot accumulate on regular points).

${ }^{6}$ the point $O$ is a flat singularity of $X \in \mathfrak{X}^{\infty}\left(\mathbb{R}^{2}\right)$ if this vector field vanishes together with its derivatives of all orders at $O$, i.e. if it has identically null Taylor expansion at that point.

${ }^{7}$ recall that two complete vector fields $X, Y \in \mathfrak{X}^{\infty}\left(\mathbb{R}^{2}\right)$ are topologically equivalent at $O$ if there are open neighbourhoods $U$ and $V$ of $O$ and a homeomorphism $\varphi: U \longrightarrow V$, fixing $O$ and carrying each maximal segment of $X$-orbit contained in $U$ onto a maximal segment of $Y$-orbit contained in $V$, preserving time orientation.
} 
$O$ cannot (with the eventual exception of some very general dynamical properties such as stability) be investigated by standard differential methods $\left(C^{r \geq 1}\right.$ coordinate changes, blow-up desingularizations, etc). This shows that already in $\mathbb{R}^{2}$, there is a continuum of distinct possible topological $C^{\infty}$ flow behaviours near an isolated singularity $O$, that are practically left in the darkness by "analytic" methods, and in such cases there seems to be no much alternative to what can be learned from the topological-dynamical approach.

This paper is organized as follows: after the preliminaries of section 2 (giving special emphasis to Hausdorff metric concepts), a provisional version of the main result (Theorem 1) is presented in section 3. Instead of the twenty eight cases of the full statement, only seventeen cases are distinguished. The last of these, condition $\mathrm{H}$ (occurrence of orbits of infinite height arbitrarily near but outside the compact invariant in question $)^{8}$ encompasses 12 distinct cases of the full statement, requiring the introduction of certain denumerable collections of orbits displaying a kind of "fractal-like" structure with respect to orbital limit relations. These collections ( $X$-trees, $X$ - $\alpha$ shells and $X$ - $\omega$ shells), capturing essential features of the dynamical complexity of this strange and beautiful phenomenon, are fully presented in section 4 . Still on section 3, some important remarks concerning the meaning and scope of application of Theorem 1 (particularly in the context of flows on manifolds), as well as some interesting consequences for the topological structure of the set of all compact minimal sets of the flow, are given. Twelve lemmas, some of them dynamically significant on their own, are presented in section 5. Lemma 2 is a version of the above mentioned Ura-Kimura-Bhatia Theorem, a proof of it being included for the sake of completeness. The main result of this paper, Theorem 1, is fully stated in section 6. As a principal corollary, we obtain a simple characterization of the topological Hausdorff structure of the set of all compact minimal sets of the flow $\operatorname{CMin}(M)$ (Theorem 2). The question of existence of flows displaying, near a compact invariant set, the types of dynamical behaviour foreseen in Theorem 1 is addressed in section 7. Examples are actually constructed showing the possibility of independent realization of some of the cases susceptible of raising greater doubts, namely those involving the occurrence of orbits of infinite height. As already mentioned, a positive answer to the question of existence of independent realizations for all the twenty eight cases will be given in [12] under quite favourable smoothness conditions. Finally a proof of Lemma 7 is presented in section 8. This result, crucial for the present work, shows that in a certain sense, the "local cardinality" of an open and dense in itself (with respect to the Hausdorff metric) nonvoid set of compact minimal sets behaves as that of (nonvoid) perfect subsets of $\mathbb{R}$.

\footnotetext{
${ }^{8}$ see condition VI on p. 3.
} 


\section{DEFINITIONS AND BASIC RESULTS.}

Let $M$ be a metric space with a $C^{0}$ flow $\theta: \mathbb{R} \times M \longrightarrow M$ and $K$ a compact, invariant, proper subset of $M$ (we denote "the flow $\theta$ on $M$ " by $(M, \theta))$. A minimal set of $(M, \theta)$ is a nonvoid, closed, invariant subset of $M$ that contains no proper subset satisfying these three conditions i.e. an orbit closure that contains no smaller one. We reserve the expression periodic orbit to orbits $\mathscr{O}(x)$ for which $\{t \in \mathbb{R}: \theta(t, x)=x\}=\lambda \mathbb{Z}$, for some $\lambda>0$. In this case, the unique $\lambda>0$ satisfying that identity is the period of $\mathscr{O}(x)$ (of the periodic point $x$ ). A minimal set that is neither an equilibrium orbit ${ }^{9}$ nor a periodic orbit is called an aperiodic minimal.

Definitions. Let $M, \theta, K$ as above and $x \in M, X \subset M$.

$$
\begin{array}{ll}
\mathscr{N}_{X}:=\text { the set of neighborhoods of } X \text { in } M . \\
\mathscr{O}(x):=\{\theta(t, x): t \in \mathbb{R}\} & \text { the orbit of } x \\
\mathscr{O}^{+}(x):=\{\theta(t, x): t \geq 0\} & \text { the positive (half) orbit of } x \\
\mathscr{O}(X):=\bigcup_{x \in X} \mathscr{O}(x) & \text { the orbital saturation of } X \\
\mathscr{O}^{+}(X):=\bigcup_{x \in X} \mathscr{O}^{+}(x) & \text { the positive orbital saturation of } X \\
\operatorname{Orb}(X):=\{\mathscr{O}(x): \mathscr{O}(x) \subset X\} & \text { the set of orbits contained in } X
\end{array}
$$

$\mathscr{O}^{-}(x)$ and $\mathscr{O}^{-}(X)$ are the negative concepts corresponding to $\mathscr{O}^{+}(x)$ and $\mathscr{O}^{+}(X)$. When dealing with a unique flow $\theta$ we write $x_{t}$ for $\theta(t, x)$.

$$
\begin{aligned}
& \omega(x):=\bigcap_{t>0} \operatorname{cl} \mathscr{O}^{+}\left(x_{t}\right) \text { the } \omega \text {-limit set of } x . \\
& \alpha(x):=\bigcap_{t<0} \operatorname{cl} \mathscr{O}^{-}\left(x_{t}\right) \text { the } \alpha \text {-limit set of } x .
\end{aligned}
$$

For any orbit $\gamma=\mathscr{O}(x)$, we define $\alpha(\gamma):=\alpha(x), \omega(\gamma):=\omega(x)$ since points in the same orbit have the same $\alpha$-limit and $\omega$-limit sets.

$$
\begin{aligned}
& B^{+}(K):=\{x \in M: \emptyset \neq \omega(x) \subset K\} \\
& B^{-}(K):=\{x \in M: \emptyset \neq \alpha(x) \subset K\} \\
& A^{+}(K):=\{x \in M: \emptyset \neq \omega(x) \cap K \neq \omega(x)\} \\
& A^{-}(K):=\{x \in M: \emptyset \neq \alpha(x) \cap K \neq \alpha(x)\}
\end{aligned}
$$

i.e. $A^{+}(K)\left(A^{-}(K)\right)$ is the set of points of $M$ whose $\omega$-limit $(\alpha$-limit $)$ set intercepts both $K$ and $M \backslash K$. We say $K$ is:

\footnotetext{
${ }^{9}$ the orbit of an equilibrium point $z$ i.e a singleton $\{z\}=\{\theta(t, z): t \in \mathbb{R}\}$.
} 
- stable if for any $U \in \mathscr{N}_{K}$ there is a $V \in \mathscr{N}_{K}$ such that $\mathscr{O}^{+}(V) \subset U$.

- negatively stable if for any $U \in \mathscr{N}_{K}$ there is a $V \in \mathscr{N}_{K}$ such that $\mathscr{O}^{-}(V) \subset U$.

- bi-stable if it is both stable and negatively stable.

- bi-stable in relation to $N \subset M$ if for any $U \in \mathscr{N}_{K}$ there is a $V \in \mathscr{N}_{K}$ such that $\mathscr{O}(N \cap V) \subset U$ i.e. given $U \in \mathscr{N}_{K}$, any point $x \in N$ at a sufficiently small distance from $K$ has its orbit entirely contained in $U$.

- an attractor ${ }^{10}$ if it is stable and $B^{+}(K) \in \mathscr{N}_{K}$.

- a repeller if it negatively stable and $B^{-}(K) \in \mathscr{N}_{K}$ i.e if it is an attractor in the time reversal flow $\phi(t, x)=\theta(-t, x)$.

- stagnan $t^{11}$ if there are points $x, y \in M \backslash K$ such that $\emptyset \neq \alpha(x) \subset K$ and $\emptyset \neq \omega(y) \subset K$.

- isolated from minimal sets if there is a $U \in \mathscr{N}_{K}$ such that $U \backslash K$ contains no minimal set of $(M, \theta)$. We also use the abridged terminology isolated from minimals. If $K$ is itself a minimal set then we say that $K$ is an isolated minimal (set).

\section{Definitions.}

$\mathrm{C}(X) \quad:=$ the set of nonvoid, compact subsets of $X$.

$\operatorname{Ci}(X) \quad:=$ the set of nonvoid, compact, invariant subsets of $X$.

$\mathfrak{S}(X) \quad:=$ the set of nonvoid, compact, connected, invariant subsets of $X$.

$\mathrm{CMin}(X):=$ the set of compact minimal sets contained in $X$.

$\mathrm{Eq}(X) \quad:=\{\{x\}: x \in M$ and $\theta(t, x)=x$ for all $t \in \mathbb{R}\}=$

$=$ the set of equilibrium orbits contained in $X$.

$\operatorname{Per}(X) \quad:=$ the set of periodic orbits contained in $X$.

$\operatorname{Am}(X) \quad:=$ the set of compact aperiodic minimal sets contained in $X$.

$\mathrm{C}(M)$ and its subsets are naturally endowed with the Hausdorff metric $d_{H}$. To emphasise that this metric is the one in question, we employ the expressions $d_{H}$-open, $d_{H}$-closed, $d_{H}$-near, $d_{H}$-converges $\left(\stackrel{d_{H}}{\longrightarrow}\right), d_{H}$-isolated, etc. 12 A set $\mathfrak{A} \subset \mathrm{C}(M) \quad d_{H}$-accumulates in $\mathfrak{B} \subset \mathrm{C}(M)$ if $\left(\operatorname{cl}_{H} \mathfrak{A}\right) \cap \mathfrak{B} \neq \emptyset$. A sequence $X_{n} \in \mathrm{C}(M) \quad d_{H}$-accumulates in $\mathfrak{B} \subset \mathrm{C}(M)$ if it has a subsequence $d_{H}$-converging to some $X \in \mathfrak{B}$. Working primarily within the Hausdorff metric, we shall deal essentially with equilibrium orbits rather than with equilibria. Note, however, that the set $E$ of equilibria of the flow, endowed with the metric

\footnotetext{
${ }^{10}$ If $K$ is an attractor then it is easily seen that $B^{+}(K)$ is an invariant, open subset of $M$. Idem for $B^{-}(K)$ if $K$ is a repeller.

11 we shall often use the expression non-stagnant instead of not stagnant.

12 Metric concepts in $\left(\mathrm{C}(M), d_{H}\right)$ are distinguished from the corresponding concepts in $(M, d)$ by the subscript ${ }_{H}$ e.g.. $B_{H}(X, \varepsilon):=\left\{Y \in \mathrm{C}(M): d_{H}(X, Y)<\varepsilon\right\} ;$ analogously, closure, boundary and interior are denoted by $\mathrm{cl}_{H}, \mathrm{bd}_{H}$, int $_{H}$.
} 
$d$ of $M$, is isometric to the metric space $\left[\mathrm{Eq}(M), d_{H}\right]$ via the canonical map $e \longrightarrow\{e\}$. The following classical result, originally proved in the context of convex body theory, is of central importance to the present work:

Blaschke Theorem: If $[N, d]$ is a compact metric space then so is $\left[\mathrm{C}(N), d_{H}\right]$.

where $\mathrm{C}(N)$ is the set of nonvoid, compact subsets of $N$ (see e.g. [3], p.253). If $N$ is a compact metric space and $\mathfrak{C}$ is a $d_{H}$-closed (and thus compact) subset of $\mathrm{C}(N)$, then the consequent possibility of selecting from a given sequence $\Lambda_{n} \in \mathfrak{C}$, a subsequence $d_{H}$-converging to some $\Lambda \in \mathfrak{C}$, will be referred as Blaschke Principle. To avoid double indices, we will often suppose that the selected subsequence is $\left(\Lambda_{n}\right)$ itself. Again in the metric space $M$, if $N \subset M$ is compact then the continuity of the flow implies that $\mathrm{Ci}(N)$ is $d_{H}$-closed in $\mathrm{C}(N)$ and thus compact; a simple argumen 13 shows that $\operatorname{Cc}(N)$, the set of nonvoid, compact, connected subsets of $N$ is also compact, hence $\mathfrak{S}(N)=$ $\mathrm{Ci}(N) \cap \mathrm{Cc}(N)$ is compact. Observe that while $\mathrm{Ci}(N), \operatorname{Cc}(N), \mathfrak{S}(N)$ and $\mathrm{Eq}(N)$ are $d_{H}$-closed in $\mathrm{C}(N)$ and thus compact, $\operatorname{CMin}(N), \operatorname{Per}(N)$ and $\operatorname{Am}(N)$ in general are not. Note that $\operatorname{CMin}(N)=\operatorname{Eq}(N) \sqcup \operatorname{Per}(N) \sqcup \operatorname{Am}(N) \subset$ $\mathfrak{S}(N) \subset \mathrm{C}(N) \quad(\sqcup$ denotes disjoint union $)$.

Remark. The reader should keep in mind the following basic facts as they will often be implicitly used without mention. Suppose $N \subset M$ is compact. If $\mathscr{O}^{+}(x) \subset N$ then $\omega(x)$ and $\operatorname{cl} \mathscr{O}^{+}(x)=\mathscr{O}^{+}(x) \cup \omega(x)$ both belong to $\mathfrak{S}(N)$ and in particular are nonvoid. Analogue fact holds for $\mathscr{O}^{-}(x), \alpha(x)$ and $\operatorname{cl} \mathscr{O}^{-}(x)$ when $\mathscr{O}^{-}(x) \subset N$. Also $\gamma \in \operatorname{Orb}(N)$ implies $\operatorname{cl} \gamma=\gamma \cup \alpha(\gamma) \cup$ $\omega(x) \in \mathfrak{S}(N)$. If $N$ is a nonvoid, compact invariant set then it contains at least one compact minimal set of the flow. If $X$ is a minimal set and $K$ is a closed invariant set, then either $X \subset K$ or $X \subset M \backslash K$, since the set of closed invariant sets is closed under interceptions. If $N \subset M$ is invariant then $\operatorname{cl} N$, bd $N$ and int $N$ (respectively, topological closure, boundary and interior of $N$ ) are also invariant.

Definitions. A set $\mathfrak{C} \subset \mathrm{C}(M)$ is $d_{H}$-dense in itself if every $\Lambda \in \mathfrak{C}$ is not $d_{H}$ - isolated in $\mathfrak{C}$ i.e. if $\Lambda \in \mathrm{cl}_{H}(\mathfrak{C} \backslash\{\Lambda\})$, for all $\Lambda \in \mathfrak{C}$. $\Lambda \in \mathrm{C}(M)$ is a $\mathfrak{c}$-condensation element of $\mathfrak{C}$ if for every $\varepsilon>0$

$$
\#\left(B_{H}(\Lambda, \varepsilon) \cap \mathfrak{C}\right) \geq \mathfrak{c}
$$

\footnotetext{
${ }^{13}$ Suppose $Y_{n} \in \mathrm{Cc}(N)$ and $Y_{n} \longrightarrow X$. If $X \in \mathrm{C}(N) \backslash \operatorname{Cc}(N)$ then $X=X_{0} \cup X_{1}$ where $X_{0}, X_{1}$ are disjoint, nonvoid compacts. Letting $\lambda:=\operatorname{dist}\left(X_{0}, X_{1}\right) / 2$, because of its connectedness, each $Y_{n}$ has at least one point $z_{n}$ in the compact $S\left(X_{0}, \lambda\right):=\left\{y \in M: \operatorname{dist}\left(y, X_{0}\right)=\lambda\right\}$. Taking a convergent subsequence $z_{n_{j}} \longrightarrow z \in S\left(X_{0}, \lambda\right)$ it follows that $z \in \lim Y_{n}=X=X_{0} \cup X_{1}$ which is absurd in virtue of the definition of $\lambda\left(\right.$ as $\left.S\left(X_{0}, \lambda\right) \cap X_{0}=\emptyset=S\left(X_{0}, \lambda\right) \cap X_{1}\right)$.
} 
where $\mathfrak{c}$ denotes the cardinal of the continuum. A set $\mathfrak{C} \subset \mathrm{C}(M)$ is $\mathfrak{c}$-dense in itself if every $\Lambda \in \mathfrak{C}$ is a $\mathfrak{c}$-condensation element of $\mathfrak{C}$.

(note that in this paper, except if otherwise mentioned, the expression $\mathfrak{c}$-dense in itself always respects to the Hausdorff metric $d_{H}$ and the same applies to $\mathfrak{c}$-condensation element).

Important remark. If $M$ is a locally compact, connected metric space, then $M$ is necessarily separable (see e.g. [9], p.278) and thus has at most a continuum of points ${ }^{14}$ Therefore there is, at most, a continuum of orbits in the flow $(M, \theta)$ and also, at most, a continuum of minimal sets (distinct minimal sets are disjoint), thus if $\mathfrak{C} \subset \mathrm{CMin}(M)$ then the inequality above reduces to

$$
\#\left(B_{H}(\Lambda, \varepsilon) \cap \mathfrak{C}\right)=\mathfrak{c}
$$

Hence for such phase space $M$, a c-dense in itself set of compact minimal sets is either empty or has the cardinal of the continuum. In chapter 5 (Lemma 6) we shall actually see that a set of compact minimal sets $\mathfrak{C} \subset \operatorname{CMin}(M)$ is $\mathfrak{c}$-dense in itself iff every neighbourhood $U_{\Lambda} \subset M$ of each $\Lambda \in \mathfrak{C}$ contains a continuum of elements of $\mathfrak{C}$, showing that in this particularly important case, we may actually think in terms of the simpler metric $d$ of $M$, instead of the Hausdorff metric $d_{H}$ of $\mathrm{C}(M)$.

Definition. For each $\mathfrak{C} \subset 2^{M}$ ( $=$ the set of subsets of $\left.M\right)$ and $A \subset M$,

$$
\begin{aligned}
& \mathfrak{C}^{*}:=\bigcup \mathfrak{C}=\bigcup_{\Gamma \in \mathfrak{C}} \Gamma \quad \text { (the set of points of } M \text { belonging to elements of } \mathfrak{C} \text { ). } \\
& \mathfrak{C}(A):=\{X \in \mathfrak{C}: X \subset A\} \quad \text { (the set of elements of } \mathfrak{C} \text { contained in } A \text { ). }
\end{aligned}
$$

Definition. (metric concept on $M$ ) Given any two nonvoid sets $X, Y \subset M$,

$$
|Y|_{X}:=\sup \{\operatorname{dist}(y, X): y \in Y\} \in[0,+\infty]
$$

If we naturally identify $B(X,+\infty)$ with $M$, then

$$
|Y|_{X}=\inf \{\delta \in[0,+\infty]: Y \subset B(X, \delta)\}
$$

\section{PRESENTATION OF THE MAIN RESULT.}

We now give the reader an approximate idea of the main result of this article (Theorem 1) which is fully stated and proved in section 6. Roughly speaking,

\footnotetext{
${ }^{14}$ Let $N \subset M$ be a countable dense subset. We can associate with each $z \in M$ a distinct sequence $x_{n} \in N$ converging to $z$. Since the cardinal of the set of all sequences of points of $N$ is at most $\#\left(\mathbb{N}^{\mathbb{N}}\right)=\# \mathbb{R}=\mathfrak{c}$, it follows that $\# M \leq \mathfrak{c}$ i.e. $M$ has, at most, a continuum of points.
} 
it shows that if $K$ is a compact, invariant, proper subset of a $C^{0}$ flow on a locally compact, connected metric space $M$, then at least one, out of twenty eight relevant dynamical phenomena, will necessarily occur near $K$. The reader is warned that the result presented bellow is weaker than Theorem 1. However, this is the best that can be done without the introduction of certain special denumerable collections of orbits (called $X$-trees, $X$ - $\alpha$ shells and $X$ - $\omega$ shells) that will be presented in the next section. In order to simplify the exposition, we shall distribute the 28 cases among seventeen conditions. The 16 cases corresponding to conditions $\mathbf{A}$ to $\mathbf{G . 4}$ will be presented in a near definitive form. The remaining 12 cases, corresponding to condition $\mathbf{H}$, require the introduction of the aforementioned collections of orbits and are postponed until section 6 . Notwithstanding, we shall give bellow an idea of the kind of dynamical phenomenon that is necessarily encountered if conditions A to G.4 fail. Here we will put emphasis on a "geometric flavoured" and rather descriptive enunciation, in detriment of concision and elegance. Whenever it is possible, Hausdorff $d_{H}$ metric properties are reformulated in terms of the simpler and more intuitive metric $d$ of $M$ (using lemma 6 of section 5). This actually results in a longer statement than that of the stronger version of section 6 . There, using appropriate Hausdorff metric terminology, a quite sharp, but perhaps at a first sight less illuminating, presentation is achieved.
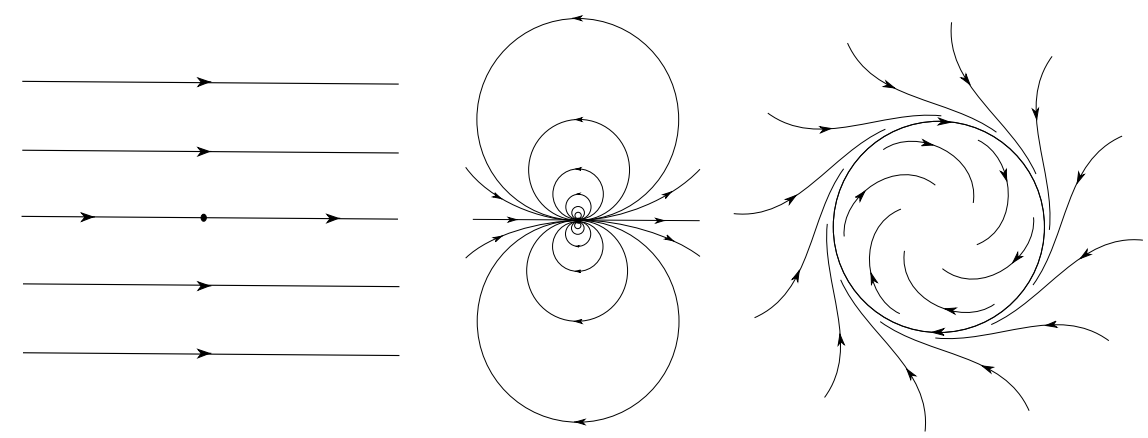

Fig. 3: Examples of isolated from minimals and stagnant compact invariant sets on $\mathbb{S}^{2}$. (Left) fake saddle equilibrium orbit. (Right) periodic orbit attracting on one side and repelling on the other (periodic orbit of saddle-node type).

Let $M$ be a locally compact, connected metric space with a $C^{0}$ flow $\theta$. Consider the following three propositions where the variable $X$ assumes values in the set $\operatorname{Ci}(M)$ of nonvoid, compact, invariant subsets of $M$ :
1.X $\quad X$ is an attractor
2.X $X$ is a repeller
3.X $X$ is isolated from minimals and stagnant 
Observe that, due to the connectedness of $M$, each compact, invariant, proper subset of $M$ may satisfy, at most, one of the above conditions: as $M$ is connected, if $X \in \operatorname{Ci}(M) \backslash\{M\}$ is an attractor then $B^{+}(X) \backslash X \neq \emptyset$ since $X$ is a closed proper subset of $M$ and $B^{+}(X)$ is an open neighbourhood of $X$. Therefore there are actually points $x \in M \backslash X$ such that $\omega(x) \subset X$. An analogue argument shows that if $X$ is a repeller then there are points $y \in M \backslash X$ such that $\alpha(y) \subset X$. Thus an attractor $X$ can neither be a repeller nor stagnant since both these conditions contradict the stability of $X$. Analogue fact holds if $X$ is a repeller. Isolated from minimals and stagnant compact, invariant sets play an important role in the present work. In Differentiable Dynamics, typical, dynamically distinct examples are given by saddle, fake saddle and saddle-node equilibrium orbits and by hyperbolic saddle periodic orbits. 15

Weak (provisional) version of Theorem 1:

Let $M$ be a locally compact, connected metric space with a $C^{0}$ flow $\theta$ and $K$ a compact, invariant, proper subset of $M$. Then:

either

A. $K$ is an attractor

or

B. $K$ is a repeller

or

C. $\quad K$ is isolated from minimals and stagnant
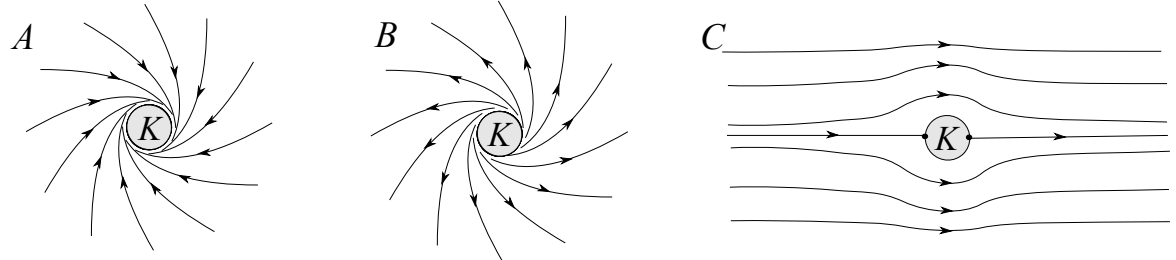

Fig. 4:

\footnotetext{
15 Another instructive example is given by the orbit of the equilibrium $(0, \ldots, 0,1) \in \mathbb{S}^{n} \subset$ $\mathbb{R}^{n+1}$ in the compactification of the flow on $\mathbb{S}^{n} \backslash\{(0, \ldots, 0,1)\}$ induced, via the inverse stereographic projection, by the constant vector field $\frac{\partial}{\partial x_{1}}$ on $\mathbb{R}^{n}$ (see fig.3, centre, for the case $n=2$ ). A more subtle example is given by the exceptional minimal set on the celebrated Denjoy $C^{1}$ flow on $\mathbb{T}^{2}$.
} 
or at least one of the following nine conditions holds:

D. $i \quad 1 \leq i \leq 3$

there is a sequence of equilibria $e_{n} \in M \backslash K$, converging to an equilibrium $q \in \operatorname{bd} K$ and such that condition $i . X$ is satisfied by all equilibrium orbits $\left\{e_{n}\right\}$.
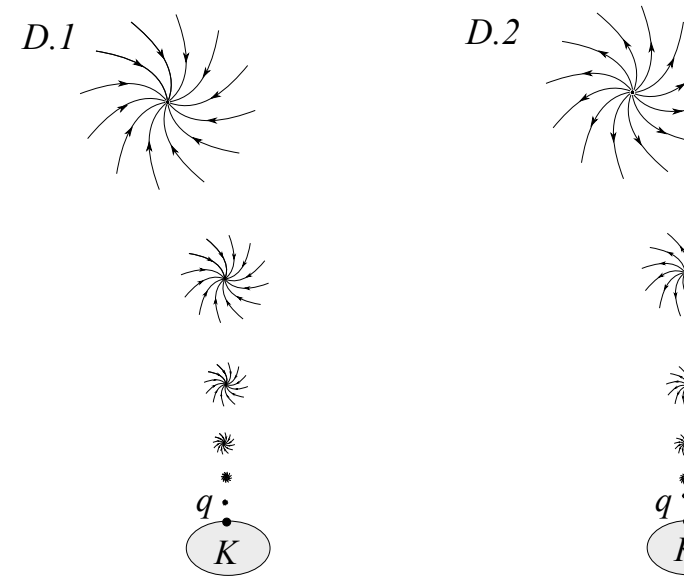

D.3
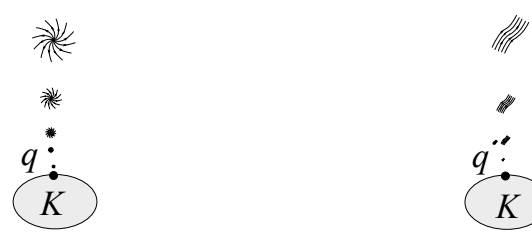

Fig. 5:

E.i $\quad 1 \leq i \leq 3$

there is a sequence of periodic orbits $\gamma_{n} \subset M \backslash K, \quad d_{H}$-converging to some nonvoid, compact, connected, invariant set $Q \subset$ bd $K$ and such that condition i.X is satisfied by all $\gamma_{n}$.
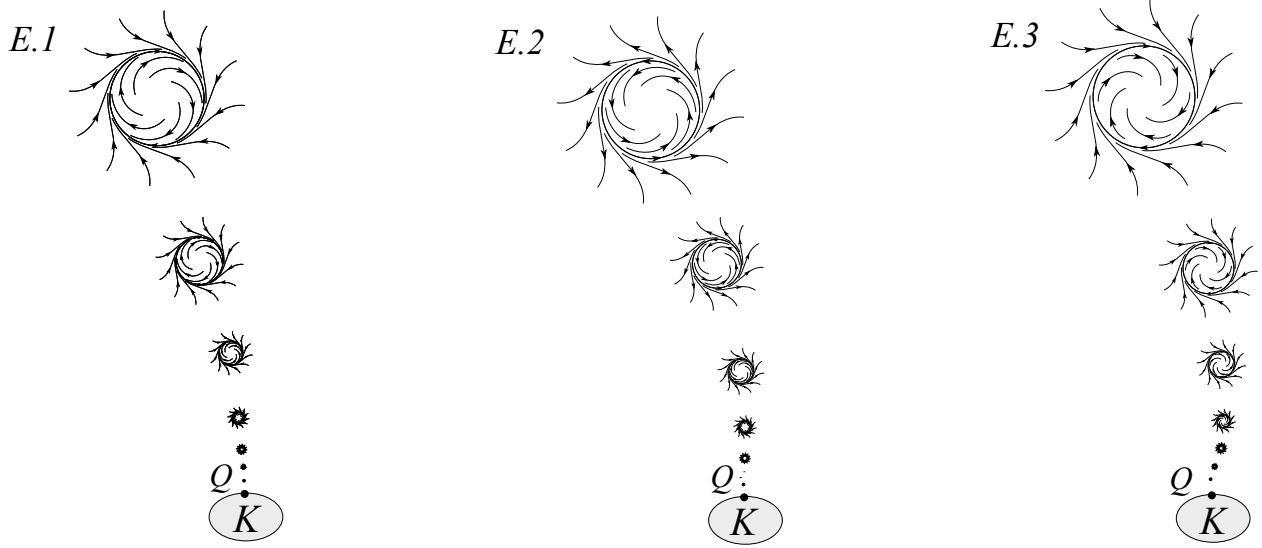

Fig. 6: 


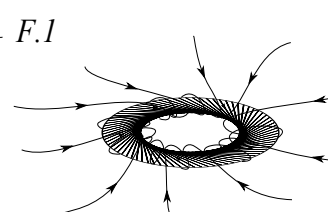

F.2

F.3
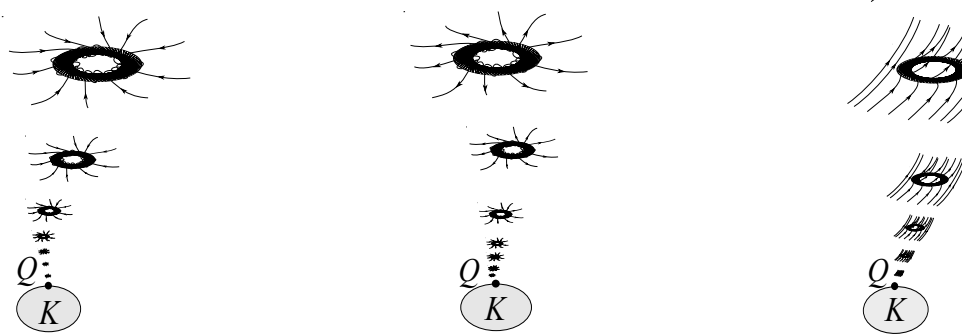

Fig. 7: this three examples show sequences of 2-tori carrying irrational linear subflows.

F.i $1 \leq i \leq 3$

there is a sequence of compact aperiodic minimal sets $\Gamma_{n} \subset M \backslash K, d_{H}$-converging to some nonvoid, compact, connected, invariant set $Q \subset$ bd $K$ and such that condition i.X is satisfied by all $\Gamma_{n}$.

G.1

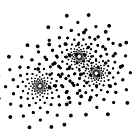

G.2

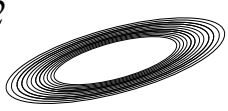

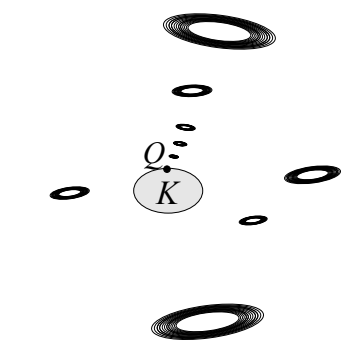

G.3

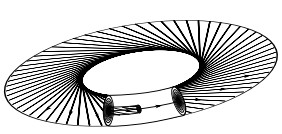

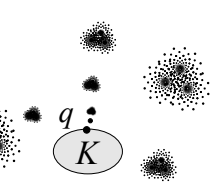

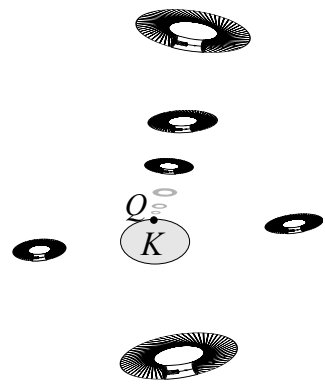

Fig. 8: figure G.3 shows nested continua of compact aperiodic minimal sets (nested 2-tori carrying irrational linear subflows).

or

$G$. there is an open neighbourhood $U$ of $K$ such that any neighbourhood of a compact minimal set $\Lambda \subset U \backslash K$ contains a continuum of compact minimal sets. Moreover, any $V \in \mathscr{N}_{K}$ actually contains a continuum of compact minimal sets disjoint from $K$, since in addition, at least one of the following four conditions holds: 
G.4
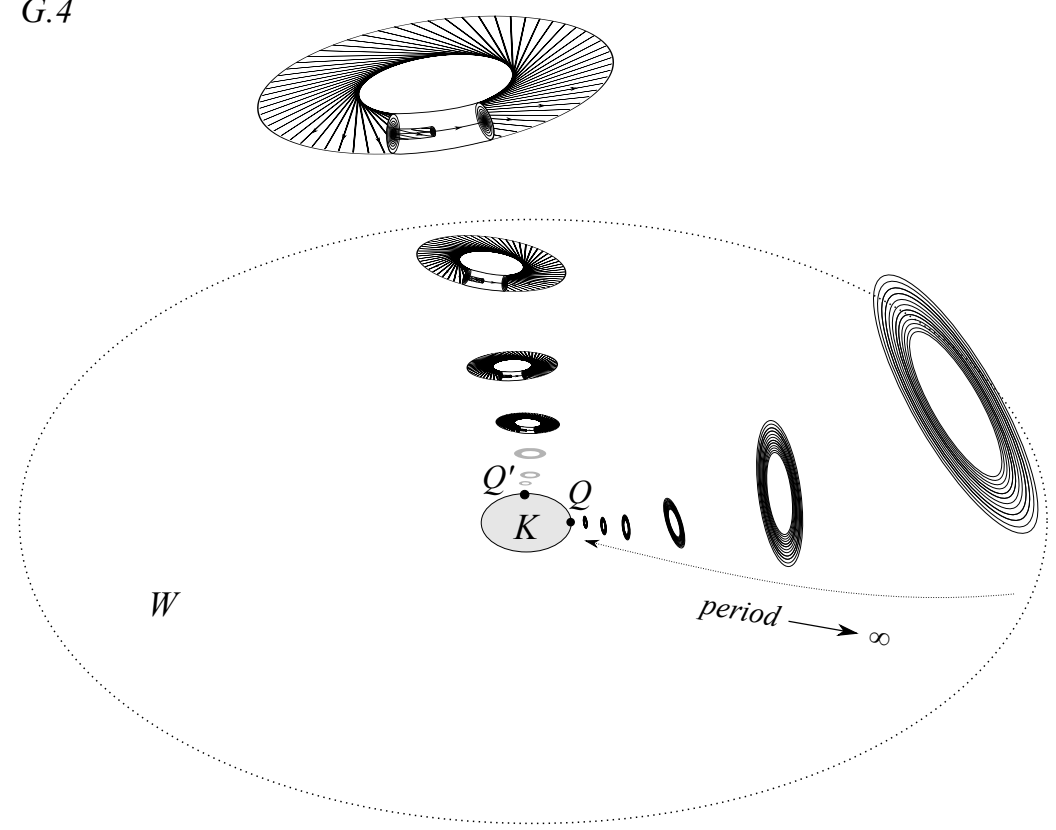

Fig. 9:

G.1 any neighbourhood of an equilibrium $z \in U \backslash K$ contains a continuum of equilibria $e \in U \backslash K$, and there is a sequence of these converging to some equilibrium $q \in \mathrm{bd} K$.

G.2 any neighbourhood of a periodic orbit $\gamma \subset U \backslash K$ contains a continuum of periodic orbits $\zeta \subset U \backslash K$, and there is a sequence of these $d_{H^{-}}$ converging to some nonvoid, compact, connected, invariant set $Q \subset \mathrm{bd} K$.

G.3 any neighbourhood of a compact aperiodic minimal set $\Gamma \subset U \backslash K$ contains a continuum of compact aperiodic minimal sets $\Lambda \subset U \backslash K$, and there is a sequence of these $d_{H}$-converging to some nonvoid, compact, connected, invariant set $Q \subset$ bd $K$.

(hence in cases G.1, G.2 and G.3 not only every neighbourhood $V$ of $K$ contains, respectively, a continuum of equilibria, a continuum of periodic orbits, a continuum of compact aperiodic minimal sets, all disjoint from $K$, but also, if $V$ is sufficiently small, then, roughly speaking, we find on $V \backslash K$ a kind of "local super-abundance" of the respective types of compact minimal sets).

G.4 there is a set $P$ of periodic orbits contained in $U \backslash K$ and a set $A$ of compact aperiodic minimal sets contained in $U \backslash K$ such that the following four conditions hold:

.1 there are sequences $\gamma_{n} \in P$ and $\Gamma_{n} \in A, d_{H}$-converging, respectively, to some nonvoid, compact, connected, invariant sets $Q, Q^{\prime} \subset$ bd $K$. 
.2 every neighbourhood $D$ of a periodic orbit $\gamma \in P$ (resp. of a compact aperiodic minimal set $\Gamma \in A$ ) contains a continuum of periodic orbits (resp. of compact aperiodic minimal sets) and if $D$ is sufficiently small, all of them belong to $P$ (resp. to A).

(hence not only every open neighbourhood $V$ of $K$ contains both a continuum $P(V)$ of periodic orbits and a continuum $A(V)$ of compact aperiodic minimal sets, all disjoint from $K$, but also, roughly speaking, both $P(V)$ and $A(V)$ display a kind of "local super-abundance" of the respective types of compact minimal sets).

$.3 \quad K$ is bi-stable in relation to $P^{*}=\bigcup_{\gamma \in P} \gamma$ and $A^{*}=\bigcup_{\Gamma \in A} \Gamma$, i.e given any neighbourhood $W$ of $K$, all periodic orbits/compact aperiodic minimal sets $\Lambda \in P \sqcup A$ at a sufficiently small distance from $K$ are contained in $W$.

.4 given any $n \geq 1$, all periodic orbits $\gamma \in P$ contained in a sufficiently small neighbourhood $V$ of $K$ have period $>n$ (note that by. 1 and .2 there is always a continuum of periodic orbits $\gamma \in P$ on $V \backslash K)$.

(hence, arbitrarily near $K$ there is a continuum of periodic orbits disjoint from $K$ having periods larger than any prescribed value).
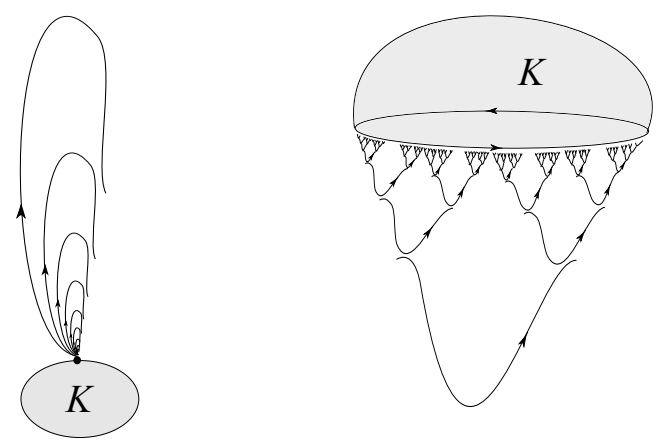

Fig. 10: for other figures illustrating case $\mathbf{H}$ see figs. 13 to 15 .

If none of the sixteen conditions $\boldsymbol{A}$ to $\boldsymbol{G . 4}$ holds, then any neighbourhood of $K$ will contain orbits of infinite height (see fig. 10), more precisely,

H. given any $U \in \mathscr{N}_{K}$, there is a sequence of orbits $\gamma_{n} \subset U \backslash K$ such that

$$
\operatorname{cl} \gamma_{1} \supsetneq \operatorname{cl} \gamma_{2} \supsetneq \cdots \cdots \supsetneq \operatorname{cl} \gamma_{n} \supsetneq \cdots \cdots
$$

In condition $\mathbf{H}$ we shall distinguish another 12 relevant cases, making up a total of $28=16+12$ cases in all. Observe that the occurrence of the above strict inclusion chain is an invulgar dynamical phenomenon. It is in deep contrast to what happens, for example, in the case of an aperiodic minimal set $\Lambda$ of a flow 
on a compact metric space (as it is well known, for any $x$ belonging to such $\Lambda, \alpha(x)=\omega(x)=\operatorname{cl} \mathscr{O}(x)=\Lambda)$.

Important remark: the reader should keep in mind the following two elementary facts about locally compact metric spaces $M$ :

- any neighbourhood of a compact set $K \subset M$ contains a compact ball $B[K, \delta]$, for some $\delta>0$.

- every open/closed subset of $M$ is also locally compact.

Remark 1. Before entering the proof of Theorem 1, we make a few brief comments concerning its applicability in the context of Differentiable Dynamics and also mention some of its consequences for the topological structure of the set $\mathrm{CMin}(M)$ of all compact minimal sets of the flow, endowed with the Hausdorff metric $d_{H}$.

Let $M$ be a (2nd countable, Hausdorff) connected $C^{m}(0 \leq m \leq \infty)$ manifold (compact or not) with a $C^{r}(0 \leq r \leq m)$ flow. Due to its topological nature, Theorem 1 (see section 6 for the complete statement) gives information, not only about the possible behaviour of the flow near each one of its compact, invariant, proper subsets (if there are any), but also, it "illuminates" the behaviour of the flow within each closed, invariant, connected subset $N$, provided it contains a compact, invariant proper subset (this is always the case if $N$ is, in addition, a non-minimal compact set, see also remark 2). Moreover, if $M$ is non-compact, then $M$ has an end-points compactification $M^{\propto}=M \sqcup E(M)$ that, roughly speaking, captures the different possible ways of going to infinite on $M$. As it is well known, besides compact, $M^{\propto}$ is connected and metrizable and the flow $\phi$ on $M$ (uniquely) extends to a $C^{0}$ flow $\theta$ on $M^{\propto}$ $\left(C^{r}\right.$ on $\left.M\right)$, with the end points $e \in E(M)$ becoming equilibria. At each end point $e \in E(M)$, not only the differentiable, but also the topological manifold structure may break (i.e. an end point may not even have a neighbourhood (in $M^{\propto}$ ) homeomorphic to $\mathbb{R}^{n}$ ). However as the extended flow is still continuous at these points, we may apply Theorem 1 to the equilibrium orbit $K=\{e\}$ of each end point $e \in E(M)$, therefore obtaining valuable insight about the possible behaviour of the original flow near each one of its "points at infinite".

Theorem 1 has many interesting consequences, some of which can already be deduced from its weak version. Bellow we give a selection of some simple Corollaries. Part II of the present work will be devoted to the investigation of more subtle implications.

Definition. Let $M$ be a metric space. A set $\mathfrak{E} \subset 2^{M}$ has elements arbitrarily near $X \subset M$ if for any $\varepsilon>0, B(X, \varepsilon)$ contains an element of $\mathfrak{E}$ (i.e. $\mathfrak{E}(B(X, \varepsilon)) \neq \emptyset)$. In this case we also say that $X$ has elements of $\mathfrak{E}$ arbitrarily nearby. More restrictively, $\mathfrak{E}$ has elements arbitrarily near (but) outside $X$ if 
for any $\varepsilon>0, \quad \mathfrak{E}(B(X, \varepsilon) \backslash X) \neq \emptyset$. We also use the expression $X$ has elements of $\mathfrak{E}$ outside arbitrarily nearby.

Observe that the last two concepts are defined using the metric of $M$ and should not be confused with $d_{H}$-nearness ${ }^{16}$ From Theorem 1 we get the following immediate consequences:

Corollary 1. Let $M$ be a locally compact, connected metric space with a $C^{0}$ flow. Then every nonvoid, compact invariant set, isolated from minimals and having no orbits of infinite height arbitrarily nearby is either an attractor or a repeller or stagnant.

Corollary 1'. Let $M$ be a locally compact, connected metric space with a $C^{0}$ flow and $K$ a (nonvoid) compact, invariant subset of $M$, isolated from minimal sets. If $K$ is neither an attractor, nor a repeller, nor stagnant, then orbits of infinite height occur arbitrarily near (but) outside $K{ }^{17}$

Ahead we shall see that if the hypothesis of Corollary 1' hold, then at least one out of three remarkable denumerable collections of orbits of infinite height (called $K$-trees, $K$ - $\alpha$ shells and $K$-wshells) will occur in the flow. Any of these implies the existence of orbits of infinite height arbitrarily near (but) outside the compact invariant $K$.

Corollary 2. Let $\theta$ be a $C^{0}$ flow on a locally compact, connected metric space having only a finite number of compact minimal sets. Then any (nonvoid) compact invariant set that is neither an attractor, nor a repeller, nor stagnant has orbits of infinite height outside arbitrarily nearby.

Again suppose $M$ is a locally compact, connected metric space with a $C^{0}$ flow $\theta$. Let

$$
\mathfrak{A}:=\{X \in \operatorname{CMin}(M): X \text { satisfies one of conditions } 1 . X \text { to } 3 . X\}
$$

that is, $\mathfrak{A}$ is the set of compact minimal sets of the flow that are either attractors, or repellers, or isolated from minimals and stagnant. The next result shows that if the compact minimal sets belonging to $\mathfrak{A}$ are not $d_{H}$-dense in $\mathrm{CMin}(M)$, then "c-abundance" of minimal sets or orbits of infinite height will occur in the flow. In the above context,

Corollary 3. Let $M$ be a locally compact, connected metric space with a $C^{0}$ flow $\theta$. If $\mathfrak{A}$ is not $d_{H}-$ dense in $\mathrm{CMin}(M)$, then there is a nonvoid, $\mathfrak{c}$-dense

\footnotetext{
${ }^{16}$ however if $M$ is a locally compact metric space with a $C^{0}$ flow, $\mathfrak{E} \subset \mathrm{Ci}(M)$ and $X \in \mathrm{CMin}(M)$, then $\mathfrak{E}$ has elements arbitrarily near $X$ implies $\mathfrak{E}$ has elements arbitrarily $d_{H}-$ near $X$ (see Lemmas 4 and 5, section 5).

${ }^{17}$ note that the if $M$ is compact then $M$ is always both an attractor and a repeller of the flow, hence $K$ must be a proper subset of $M$ anyway.
} 
in itself, $d_{H}$-open subset of $\operatorname{CMin}(M)$ or there are orbits of infinite height arbitrarily near every $Y \in \mathrm{CMin}(M) \backslash \mathrm{cl}_{H} \mathfrak{A} \neq \emptyset$.

We prove a stronger "local" result. Corollary 2 then follows letting $A=M$.

Corollary 4. Let $M$ be a locally compact, connected metric space with a $C^{0}$ flow $\theta$ and $A$ an open subset of $M$. If the set

$$
\mathfrak{A}(A)=\{X \in \operatorname{CMin}(A): X \text { satisfies one of conditions } 1 . X \text { to } 3 . X\}
$$

is not $d_{H}-$ dense in $\operatorname{CMin}(A)$, then at least one of the following two situations occurs:

a) there is a nonvoid, $\mathfrak{c}$-dense in itself, $d_{H}$-open subset of $\operatorname{CMin}(M)$ contained in $\mathrm{CMin}(A)$.

b) there are orbits of infinite height arbitrarily near every $Y \in \operatorname{CMin}(A) \backslash \operatorname{cl}_{H} \mathfrak{A}(A) \neq \emptyset$.

In particular, if there are only countably many compact minimal sets in A, then case b) takes place.

Proof. By hypothesis $A \subset M$ is open and $\Delta:=\mathrm{CMin}(A) \backslash \mathrm{cl}_{H} \mathfrak{A}(A) \neq \emptyset$, hence $\mathrm{CMin}(A)$ is a nonvoid, $d_{H}$-open subset of $\mathrm{CMin}(M) \subset \mathrm{C}(M)$ (lemma 8 , section 5). The set

$$
\Upsilon:=\{Y \in \Delta: \text { there are orbits of infinite height arbitrarily near } Y\}
$$

is clearly a $d_{H}$-closed subset of $\Delta$. Suppose $\Theta:=\Delta \backslash \Upsilon \neq \emptyset$ i.e. assume there are compact minimal sets in $\Delta$ having no orbits of infinite height arbitrarily nearby. Then $\Theta$ is a nonvoid, $d_{H}$-open subset of $\operatorname{CMin}(M)$. Let $K \in$ $\Theta \subset \mathrm{CMin}(A)$. Then since $K$ is a compact minimal set, there is an open $U \in \mathscr{N}_{K}$ with compact closure contained in $A$ and such that $U$ contains no $Y \in$ $\mathfrak{A}(A)$ (this follows from lemma 4 (section 5), as $K \in \mathrm{CMin}(A) \backslash \mathrm{cl}_{H} \mathfrak{A}(A)$ ), and also contains no orbit of infinite height (observe that these two facts together also imply that any $\Lambda \in \mathrm{CMin}(U)$ belongs to $\Theta)$. Hence none of the 13 conditions $\mathbf{A}$ to $\mathbf{F . 3}$ and $\mathbf{H}$ of the provisional version of Theorem 1 holds, thus by the same result, at least one of the 4 conditions G.1 to G.4 must take place. But any of these implies the existence of a continuum of compact minimal sets contained in every $B(K, \delta), \delta>0$ and thus, by lemma 6 (section 5 ), of a continuum of compact minimal sets in every $B_{H}(K, \varepsilon), \varepsilon>0$, and for $\varepsilon$ small enough these are contained in $U$ and thus must belong to $\Theta$. Therefore $\Theta$ is $\mathfrak{c}$-dense in itself

Obviously, every $X \in \mathfrak{A}(M)$ is $d_{H}$-isolated in $\operatorname{CMin}(M)$ (and thus $\{X\}$ is $d_{H}$-open in $\left.\operatorname{CMin}(M)\right)$, hence in the above context, 
Corollary 5. If $\theta$ is a $C^{0}$ flow on a locally compact, connected metric space $M$ with only countably many compact minimal sets and displaying no orbits of infinite height, then the set $\mathfrak{A}(M)$ is $d_{H}$-open dense in $\mathrm{CMin}(M)$.

Remark 2. Suppose $N$ is a locally compact, connected metric space endowed with a $C^{0}$ flow $\phi$ and $M$ a connected, closed invariant subset of $N$, containing a compact invariant proper subset $K$. Then Theorem 1 applies to the subflow $(M, \theta)$ where $\theta:=\left.\phi\right|_{\mathbb{R} \times M}, \quad M$ endowed with the metric of $N$. In this situation all definitions must be interpreted "within" $(M, \theta)$ i.e. as concerning this subflow (for example a nonvoid, compact invariant set $\Lambda \subset M$ may be an attractor in $(M, \theta)$ without being one in $(N, \phi))$. The next result shows that if addition the phase space $N$ is locally connected and is separated by the compact invariant set $J$, then a finner understanding of the flow behaviour near $J$ is possible.

Corollary 6. Let $N$ be a locally compact, connected and locally connected metric space with a $C^{0}$ flow and $J$ a compact, invariant proper subset of $N$. Let $D$ be a connected component of $N \backslash J$. Then Theorem 1 applies to $M:=\operatorname{cl} D, \quad \theta:=\left.\phi\right|_{\mathbb{R} \times M}, \quad K:=M \cap J$.

Roughly speaking, this result means that within the closure of each connected component $D$ of $N \backslash J$, at least one of the 28 phenomena described in Theorem 1 (see section 6 for the full statement) takes place near the compact invariant $(\mathrm{cl} D) \cap J$ (it being possible that within distinct components, different conditions hold).

Proof. Consider the collection $\Theta$ of all connected components of $N \backslash J$. Since $N$ is locally connected and $N \backslash J$ is open, every $D \in \Theta$ is open in $N$, hence it cannot be closed as $N$ is connected. On the other hand, every $D \in \Theta$ is closed in $N \backslash J$, hence $\emptyset \neq \mathrm{bd} D=(\operatorname{cl} D) \backslash D \subset J$. The invariance of each $D \in \Theta$ now follows from that of $N \backslash J$ : the orbit of a point $z \in D$ cannot pass from $D$ to a different $D^{\prime} \in \Theta$ without intercepting bd $J \subset J$, and this is impossible since $N \backslash J \supset D$ is invariant. Therefore $M:=\operatorname{cl} D$ is a nonvoid, connected, closed (and hence locally compact) invariant subset of $N$ and $K=(\operatorname{cl} D) \cap J$ is a nonvoid, compact, invariant proper subset of $M$. Define the (sub)flow $\theta:=\left.\phi\right|_{\mathbb{R} \times M}$. Now endowed with the metric of $N, M$ is a compact, connected metric space with a $C^{0}$ flow $\theta$ and $K$ is a compact, invariant (under $\theta$ ) proper subset of $M$. Theorem 1 can thus be applied to these $M, \theta$ and $K$.

Example. Let $\phi$ be a $C^{r}(r \geq 0)$ flow on $N=\mathbb{S}^{n}$ and $K \subset N$ an invariant, codimension one, compact, connected $C^{0}$ submanifold. As it is well known, by the generalized Jordan-Brouwer Separation Theorem, ${ }^{18} K$ separates the

\footnotetext{
18 J. W. Alexander, "A proof and extension of the Jordan-Brouwer separation theorem", Trans. A.M.S. 23, 333-349 (1922). Alexander's term "immersed" means $C^{0}$-embedded. Recall that this work is prior to Whitney's foundational papers on the theory of manifolds.
} 
flow into three invariant regions, $K, B$ and $A$, the last two being the connected components of $N \backslash K$, with common boundary $K$. Besides applying to $N, \phi$, $K$, Theorem 1 also applies to $M=A \sqcup K, \theta=\left.\phi\right|_{\mathbb{R} \times M}, K$ and to $M=B \sqcup K$, $\theta=\left.\phi\right|_{\mathbb{R} \times M}, K$. Moreover, if $K$ is not a minimal set, then it also applies to the (compact, connected, metric) phase space $K$, giving, in this case, information about the possible behaviour of the codimension one subflow $\theta=\left.\phi\right|_{\mathbb{R} \times K}$ near any compact, invariant, proper subset of $K$ (there is at least one). This is always the case if, for example, $K$ is the image of a $C^{0}$ embedding $\mathbb{S}^{2 m} \hookrightarrow \mathbb{S}^{2 m+1}$, $n=2 m+1$ (since such $K$ must contain an equilibrium, even if $\phi$ is only $C^{0}$ ).

\section{SPECIAL ORBITAL STRUCTURES.}

We will introduce three kinds of "orbital structures", $X$-trees, $X$ - $\alpha$ shells and $X$ - $\omega$ shells. The reason for considering these denumerable collections of orbits lies in the fact that they capture essential features of the "dynamical complexity" of those flows on which they occur. In particular, their presence implies that arbitrarily near $X$ there are orbits having limit sets of an outstanding kind.

Throughout this section, $X$ is a compact, invariant, proper subset of a $C^{0}$ flow on a locally compact metric space $M$.
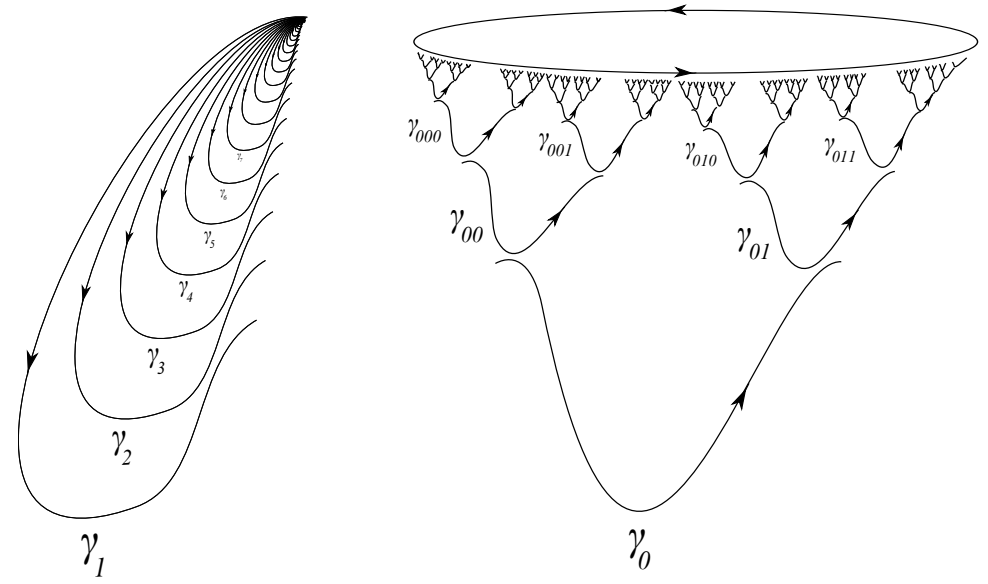

Fig. 11: (Left) a $X$ - $\omega$ shell with $X$ an equilibrium orbit. Time reversing the flow $X$ - $\alpha$ shell is obtained. (Right) a $X$-tree with $X$ a periodic orbit.

\subsection{X-trees.}

Let $\mathrm{F}:=\{0,1\}$ and $\mathrm{E}_{0}:=\{0\}$. Define

$$
\mathrm{E}_{n}:=\{0\} \times \mathrm{F}^{n}, n \geq 1 \quad \mathscr{E}:=\bigsqcup_{n \geq 0} \mathrm{E}_{n} \quad \mathrm{E}_{\infty}:=\{0\} \times \mathrm{F}^{\mathbb{N}}
$$


$\left(\mathscr{E}\right.$ and $\mathrm{E}_{\infty}$ are, respectively, the set of finite and the set of infinite sequences of 0 's and 1's with first (left) digit 0 ). Since no risk of ambiguity arises, commas and brackets are omitted in the representation of both finite and infinite sequences of 0 's and 1's e.g. we write 01 and $00 \ldots 0 \ldots \ldots$ instead of $(0,1)$ and $(0,0, \ldots, 0, \ldots \ldots)$. If $a, b \in \mathscr{E}, a b$ represents, as usual, the element of $\mathscr{E}$ obtained by adjoining $b$ to the right side of $a$. For each $v \in \mathrm{E}_{\infty}\left(v=0 c_{1} \ldots c_{n} \ldots \ldots, c_{n} \in\{0,1\}\right.$ for all $\left.n \geq 1\right)$, define $v_{0}:=0$ and $v_{n}:=0 \ldots c_{n}$ for all $n \geq 1$.

Definition. If $\gamma, \zeta \in \operatorname{Orb}(M)$, we denote $\zeta \subset \alpha(\gamma), \zeta \subset \omega(\gamma)$ and $\zeta \subset$ $\alpha(\gamma) \cup \omega(\gamma)$ by $\gamma \stackrel{0}{\succ} \zeta, \quad \gamma \succ \zeta$ and $\gamma \succ \zeta$, respectively. Note that all these three relations are transitive and $\gamma \succ^{c} \zeta$ and $\zeta \succ \xi$ implies $\gamma \succ^{c} \xi$, for $c \in\{0,1\}$.

Let $U$ be a compact neighbourhood of $X$. A $X$-tree is a pair $(\Theta, \psi)$ where $\Theta$ is a collection of orbits contained in $U \backslash X$ and $\psi$ is a surjective map

$$
\begin{aligned}
\psi: \mathscr{E} & \longrightarrow \Theta \subset \operatorname{Orb}(U \backslash X) \\
a & \longmapsto \gamma_{a}
\end{aligned}
$$

such that for any $b \in \mathscr{E}$,

$$
\begin{array}{lll}
\gamma_{b} \stackrel{0}{ } \gamma_{b 0} & \text { and } & \gamma_{b 0} \nsucc \gamma_{b} \\
\gamma_{b} & \succ \gamma_{b 1} \quad \text { and } & \gamma_{b 1} \nsucc \gamma_{b}
\end{array}
$$

and for every $v \in \mathrm{E}_{\infty}$,

$$
\left|\mathrm{cl} \gamma_{v_{n}}\right|_{X} \longrightarrow 0
$$

$\gamma_{0}$ is called the first orbit of the $X$-tree (see fig.11). Observe that (1) implies (because of the transitivity of relation $\succ$ ) that for every $v \in \mathrm{E}_{\infty}$, the sequence $\left(\gamma_{v_{n}}\right)$ is injective i.e. the $\gamma_{v_{n}}$ 's are distinct and therefore $\Theta$ is denumerable (since $\mathscr{E}$ is). $X$-trees have significant dynamical properties, some of which we single out: 19

i) every $z \in \gamma \in \Theta$ belongs to $A^{-}(X) \cap A^{+}(X)$

For each $v \in \mathrm{E}_{\infty}\left(v=0 c_{1} \cdots c_{n} \cdots \cdots, \quad c_{n} \in\{0,1\}\right.$ for all $\left.n \geq 1\right)$,

ii)

$$
\gamma_{v_{0}} \stackrel{c_{1}}{\succ} \gamma_{v_{1}} \succ^{c_{2}} \gamma_{v_{2}} \succ^{c_{3}} \cdots \cdots \cdot \succ^{c_{n}} \gamma_{v_{n}} \stackrel{c_{n+1}}{\succ} \gamma_{v_{n+1}} \stackrel{c_{n+2}}{\succ} \cdots
$$

\footnotetext{
${ }^{19}$ recall that $A^{+}(X)$ is the set of points of $M$ whose $\omega$-limit set intercepts both $X$ and $M \backslash X . \quad A^{-}(X)$ is the corresponding negative concept.
} 
and

$$
q>p \Longrightarrow \gamma_{v_{q}} \nsucc \gamma_{v_{p}}
$$

thus

$$
\operatorname{cl} \gamma_{v_{n}} \supsetneq \operatorname{cl} \gamma_{v_{n+1}} \text { for all } n \geq 0
$$

iii)

$$
\operatorname{cl} \gamma_{v_{n}} \stackrel{d_{H}}{\longrightarrow} \Lambda_{v}:=\left(\bigcap_{n \geq 1} \operatorname{cl} \gamma_{v_{n}}\right) \in \mathfrak{S}(X)
$$

Proof. i) if $z \in \gamma_{b} \in \Theta, b \in \mathscr{E}$ then $\gamma_{b} \succ^{0} \gamma_{b 0}, \gamma_{b} \succ \gamma_{b 1}$ where $\gamma_{b 0}, \gamma_{b 1} \in \Theta \subset$ $\operatorname{Orb}(U \backslash X)$, hence both the $\alpha$-limit and $\omega$-limit sets of $z$ have points outside $X$. On the other hand letting $k_{n}:=\{0\}^{n} \in \mathrm{F}^{n}$ and $l_{n}:=\{1\}^{n} \in \mathrm{F}^{n}$ it follows immediately from (1) that $\gamma_{b} \stackrel{0}{\succ} \gamma_{b k_{n}}$ and $\gamma_{b} \stackrel{1}{\succ} \gamma_{b l_{n}}$ for all $n \geq 1$; also (2) implies that both $\left|\gamma_{b k_{n}}\right|_{X}$ and $\left|\gamma_{b l_{n}}\right|_{X}^{n}$ tend to zero when $n \longrightarrow+\infty$, thus both the $\alpha$-limit and $\omega$-limit sets of $z$ intercept $X$, since these two sets are closed. ii) (3) is trivial; (4) and (5) follow from (1) because of the transitivity of $\succ$. iii) $\operatorname{cl} \gamma_{v_{n}} \in \mathfrak{S}(U)$ and $\operatorname{cl} \gamma_{v_{n+1}} \subset \operatorname{cl} \gamma_{v_{n}}$ for all $n \geq 0$, therefore by Lemma 11 (section 5), $\operatorname{cl} \gamma_{v_{n}} \stackrel{d_{H}}{\longrightarrow} \Lambda_{v} \in \mathfrak{S}(U)$ since $\mathfrak{S}(U)$ is compact (recall that $U \in \mathscr{N}_{X}$ is compact); on the other hand $\left|\operatorname{cl} \gamma_{v_{n}}\right|_{X} \longrightarrow 0$ hence $\Lambda_{v} \subset X$ and finally $\Lambda_{v} \in \mathfrak{S}(X)$.

Observe that if $(\Theta, \psi)$ is a $X$-tree, then given any $a \in \mathscr{E}$, letting

$$
\Upsilon=\left\{\gamma_{d}: d=a \text { or } d=a b, \quad b \in \mathrm{F}^{n}, n \geq 1\right\}
$$

and defining the surjective map

$$
\begin{aligned}
\phi: \mathscr{E} & \longrightarrow \Upsilon \\
0 & \longmapsto \zeta_{0}:=\gamma_{a}=\psi(a) \\
0 b & \longmapsto \zeta_{0 b}:=\gamma_{a b}=\psi(a b) \quad \text { for each } b \in \underset{n \geq 1}{\sqcup \mathrm{F}^{n}}
\end{aligned}
$$

we get a $X$-tree with first orbit $\gamma_{a}$, whose orbits are contained in $\Theta$. We call $(\Upsilon, \phi)$ a sub $X$-tree of $(\Theta, \psi)$ and commit a safe abuse of expression saying that $(\Upsilon, \phi)$ is contained in $(\Theta, \psi)$. Note that $\left|\zeta_{d}\right|_{X} \leq\left|\operatorname{cl} \zeta_{0}\right|_{X}=\left|\zeta_{0}\right|_{X}=\left|\gamma_{a}\right|_{X}$ for all $d \in \mathscr{E}$, since $\zeta_{d} \subset \zeta_{0} \cup \alpha\left(\zeta_{0}\right) \cup \omega\left(\zeta_{0}\right)=\mathrm{cl} \zeta_{0}$. Therefore, in virtue of (2), given a $X$-tree $(\Theta, \psi)$ and an $\varepsilon>0$, there is always a sub $X$-tree of $(\Theta, \psi)$ with all its orbits contained in $B(X, \varepsilon) \backslash X$.

\subsection{X- $\alpha$ shells and $X$ - $\omega$ shells.}

We define $X$ - $\omega$ shells. $X$ - $\alpha$ shells are the time symmetric concept i.e. a sequence of orbits $\left(\gamma_{n}\right)_{n \geq 1}$ is a $X$ - $\alpha$ shell if it is a $X$ - $\omega$ shell in the time 
reversal flow $\phi(t, x)=\theta(-t, x)$. Let $U$ be a compact neighbourhood of $X$. A $X$ - $\omega$ shell is a sequence of orbits $\gamma_{n} \subset U \backslash X$ satisfying the following three conditions:

- $\gamma_{n} \subset B^{-}(X)$ for every $n \geq 1$

- $\gamma_{n} \succ \gamma_{n+1}$ and $\gamma_{n+1} \nsucc \gamma_{n}$, for all $n \geq 1$

- $\left|\operatorname{cl} \gamma_{n}\right|_{X} \longrightarrow 0$

These imply ${ }^{20}$ that $\gamma_{n} \subset A^{+}(X)$ for every $n \geq 1$ and hence

$$
\gamma_{n} \subset B^{-}(X) \cap A^{+}(X) \quad \text { for every } n \geq 1
$$

Also, the sequence $\left(\gamma_{n}\right)$ is necessarily injective i.e. the $\gamma_{n}$ 's are distinct (see fig.11). Again, as in the case of $X$-trees, it is easily seen that:

$$
\begin{gathered}
\gamma_{1} \succ \gamma_{2} \succ \cdots \cdots \gamma_{n} \succ \gamma_{n+1} \succ \cdots \\
q>p \Longrightarrow \gamma_{q} \nsucc \gamma_{p} \\
\operatorname{cl} \gamma_{n} \supsetneq \operatorname{cl} \gamma_{n+1} \quad \text { for all } n \geq 1 \\
\operatorname{cl} \gamma_{n} \stackrel{d_{H}}{\longrightarrow} \Lambda:=\left(\bigcap_{n \geq 1} \operatorname{cl} \gamma_{n}\right) \in \mathfrak{S}(X)
\end{gathered}
$$

$X$ - $\alpha$ shells have exactly the same properties, interchanging $\alpha$ with $\omega$, + with - and changing $\succ^{1}$ to $\succ^{0}$ everywhere. Obviously, if $\left(\gamma_{n}\right)_{n \geq 1}$ is a $X$ - $\omega$ shell then any subsequence $\left(\gamma_{n_{i}}\right)_{i \geq 1}$ is also a $X$ - $\omega$ shell and we call it a sub $X$ - $\omega$ shell of $\left(\gamma_{n}\right)_{n \geq 1}$. Therefore, since $\left|\mathrm{cl} \gamma_{n}\right|_{X} \longrightarrow 0$, given any $\varepsilon>0$, a $X$ - $\omega$ shell always has a sub $X-\omega$ shell with all its orbits contained in $B(X, \varepsilon) \backslash X$. Analogue fact holds for $X$ - $\alpha$ shells.

\section{LEMMAS.}

The following result gives an unusual characterisation of attractors in terms of the behaviour of the negative orbits of points outside the compact invariant in question. It illustrates a topological-dynamical phenomenon that plays a key role in the present work.

Lemma 1. Let $M$ be a locally compact metric space with a $C^{0}$ flow $\theta$ and $K$ a compact, invariant, proper subset of $M$. Then $K$ is an attractor iff there is a neighbourhood $U$ of $K$ such that no point $z \in U \backslash K$ has its negative

\footnotetext{
20 clearly $\gamma_{n} \stackrel{1}{\succ} \gamma_{m}$ for every $1 \leq n<m$, thus $\omega\left(\gamma_{n}\right) \cap X \neq \emptyset$ since $\left|\operatorname{cl} \gamma_{m}\right|_{X} \longrightarrow 0$ and $\omega\left(\gamma_{n}\right)$ is closed. On the other hand, $\omega\left(\gamma_{n}\right) \not \subset X$ because $\gamma_{n+1} \subset \omega\left(\gamma_{n}\right)$ and $\gamma_{n+1} \subset M \backslash X$. Hence $\gamma_{n} \subset A^{+}(X)$.
} 
orbit $\mathscr{O}^{-}(z)$ entirely contained in $U$. Analogously, $K$ is a repeller iff there is a $U \in \mathscr{N}_{K}$ such that $z \in U \backslash K \Longrightarrow \mathscr{O}^{+}(z) \not \subset U$.

We need only to prove the characterisation of attractors in Lemma 1 since a compact, invariant set is a repeller iff it is an attractor in the time reversal flow.
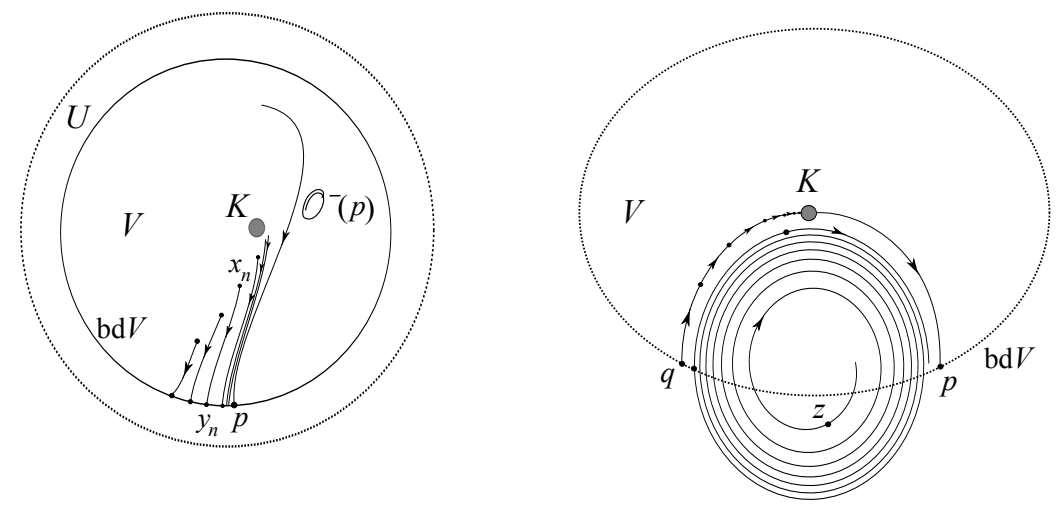

Fig. 12: (Left) Lemma 1, the non-stability of $K$ implies the existence of a negative orbit trapped in $U \backslash K$, for every $U \in \mathscr{N}_{K}$. (Right) Lemma 3.

Let $M, \theta, K$ be as in Lemma 1. The following elementary result (and the analogue fact for repellers) will be used:

Claim: in the context of lemma 1 , if $K$ is an attractor and $z \in B^{+}(K) \backslash K$ then $\alpha(z) \subset b d B^{+}(K) \subset M \backslash B^{+}(K) \cdot{ }^{21}$

Proof of Lemma 1. ( $\Longrightarrow$ ) Suppose $K$ is an attractor. Let $U$ be a compact neighbourhood of $K$ contained in the open set $B^{+}(K) \in \mathscr{N}_{K}$. Clearly $U$ and the compact bd $B^{+}(K)$ are disjoint, hence, $z \in U \backslash K \subset B^{+}(K) \backslash K \Longrightarrow$ $\mathscr{O}^{-}(z) \not \subset U$ since $\mathscr{O}^{-}(z) \subset U \Longrightarrow \emptyset \neq \alpha(z) \subset U \subset B^{+}(K)$, which contradicts $\alpha(z) \subset \mathrm{bd} B^{+}(K) \subset M \backslash B^{+}(K)$.

$(\Longleftarrow)$ Suppose $U \in \mathscr{N}_{K}$ is such that $z \in U \backslash K \Longrightarrow \mathscr{O}^{-}(z) \not \subset U$. Without loss of generality we may assume $U$ is compact 22

Claim I. $K$ is stable: Suppose the contrary. Then there is a $V_{0} \subset \mathscr{N}_{K}$ such that every neighbourhood of $K$ contains a point $x$ for which $\mathscr{O}^{+}(x) \not \subset V_{0}$ (observe

21 Suppose $z \in B^{+}(K) \backslash K$. It is easily seen that $B^{+}(K)$ is an open, invariant set hence $\alpha(z) \subset \operatorname{cl} \mathscr{O}(z) \subset \operatorname{cl} B^{+}(K)=B^{+}(K) \sqcup$ bd $B^{+}(K)$. Necessarily, $\alpha(z) \cap B^{+}(K)=\emptyset$ : otherwise there is a $y \in \alpha(z) \cap B^{+}(K)$ implying both $\emptyset \neq \omega(y) \subset K$ and $\omega(y) \subset \alpha(z) \quad(\omega(y) \subset$ $\operatorname{cl} \mathscr{O}(y) \subset \alpha(z)$, as $\alpha(z)$ is closed invariant), which by their turn imply that $\alpha(z) \cap K \neq \emptyset$, and this contradicts the stability of $K$, since $z \in M \backslash K$. Therefore $\alpha(z) \subset$ bd $B^{+}(K) \subset M \backslash B^{+}(K)$. Note however that we may have $\alpha(z)=\emptyset$. A time-symmetric argument proves the analogue fact for repellers.

22 if $U$ is not compact, take a compact $V \in \mathscr{N}_{K}$ contained in $U$ (this is always possible since $K$ is compact and $M$ is locally compact). Clearly $z \in V \backslash K \Longrightarrow \mathscr{O}^{-}(z) \not \subset V$. Let $U:=V$. 
that $x \in M \backslash K$ since $K$ is invariant). Let $V$ be a compact neighbourhood of $K$ such that $V \subset U \cap V_{0}$. Since $M \backslash K$ is invariant, the above remark implies that there are sequences $x_{n} \in V \backslash K$ and $t_{n} \in \mathbb{R}^{+}$such that:

a) $\operatorname{dist}\left(x_{n}, K\right) \longrightarrow 0$

b) $y_{n}:=\theta\left(t_{n}, x_{n}\right) \in \mathrm{bd} V$

c) $\theta\left(\left[0, t_{n}\left[\times\left\{x_{n}\right\}\right) \subset V \backslash K\right.\right.$ which is equivalent to $\theta\left(\left[-t_{n}, 0\left[\times\left\{y_{n}\right\}\right) \subset V \backslash K\right.\right.$

Since bd $V$ is compact, replacing $y_{n}$ by a convergent subsequence we may suppose $y_{n} \longrightarrow p$ for some $p \in \operatorname{bd} V \subset U \backslash K \subset M \backslash K$. We claim that $\mathscr{O}^{-}(p) \subset$ $V \subset U$. Assuming the contrary i.e. $\mathscr{O}^{-}(p) \not \subset V$, there is a $T<0$ such that $\lambda:=\operatorname{dist}\left(p_{T}, V\right)>0$. By the continuity of the flow, there is a $\delta>0$ such that

$$
z \in B(p, \delta) \Longrightarrow z_{T} \in B\left(p_{T}, \lambda / 2\right)
$$

hence for some $n_{0} \geq 1$,

$$
n>n_{0} \Longrightarrow y_{n} \in B(p, \delta) \Longrightarrow \theta\left(T, y_{n}\right) \in B\left(p_{T}, \lambda / 2\right) \subset M \backslash V
$$

But necessarily $t_{n} \longrightarrow+\infty$ since $\operatorname{dist}\left(x_{n}, K\right) \longrightarrow 0$ and $y_{n} \in \mathrm{bd} V$ (observe that if $\left(t_{n_{i}}\right)$ is a bounded subsequence of $\left(t_{n}\right)$ then $\left\{x_{n_{i}}: i \geq 1\right\} \subset \Theta:=$ $\theta([-t, 0] \times$ bd $V)$ where $t:=\sup \left\{t_{n_{i}}: i \geq 1\right\}, \Theta$ being a compact disjoint from the compact invariant $K$, hence $\operatorname{dist}\left(x_{n_{i}}, K\right) \nrightarrow 0$, a contradiction). Thus for some $n_{1}>n_{0}$,

$$
n>n_{1} \Longrightarrow\left(-t_{n}<T<0 \text { and } \theta\left(T, y_{n}\right) \notin V\right)
$$

in contradiction with c). Hence $p \in U \backslash K$ and $\mathscr{O}^{-}(p) \subset V \subset U$ in contradiction with the initial hypothesis. Therefore $K$ is stable.

Claim II. $B^{+}(K)$ is a neighbourhood of $K$ : Recall that by hypothesis $U$ is a compact neighbourhood of $K$. Since $K$ is stable, there is a $V \in \mathscr{N}_{K}$ such that $\mathscr{O}^{+}(V) \subset U$, which implies $\emptyset \neq \omega(x) \subset U$ for every $x \in U$. We claim that $V \subset B^{+}(K)$. By reductio ad absurdum, suppose there are $x \in V$ and $y \in M \backslash K$ such that $y \in \omega(x)$. Clearly

$$
\operatorname{cl} \mathscr{O}^{+}(x)=\mathscr{O}^{+}(x) \cup \omega(x) \subset \operatorname{cl} U=U
$$

hence $y \in U \backslash K$. But by hypothesis, $\mathscr{O}^{-}(y) \not \subset U$ which by its turn implies $\omega(x) \not \subset U$, since $\mathscr{O}(y) \subset \omega(x)$ (as $\omega(x)$ is an invariant set). We have reached contradiction. The proof of Lemma 1 is complete.

The following result is essentially a version of the Ura-Kimura-Bhatia Theorem for locally compact, connected metric spaces (see e.g. [1], p.114). Our proof is given within the spirit of the present work. 
Lemma 2. Let $M$ be a locally compact, connected metric space with a $C^{0}$ flow $\theta$ and $K$ a compact, invariant, proper subset of $M$. Then:

either I. $K$ is an attractor.

or II. $K$ is a repeller.

or at least one of the following two situations occurs:

III. $K$ is isolated from minimals and stagnant.

IV. given any neighbourhood $U$ of $K, U \backslash K$ contains an (entire) orbit.

Proof. If $K$ is an attractor then it is easily seen that none of conditions II, III and IV holds: clearly $B^{+}(K) \backslash K \neq \emptyset$ since $M$ is connected, $B^{+}(K)$ is an open neighbourhood of $K$ and $K$ is a closed, proper subset of $M$. Hence there is necessarily a $x \in M \backslash K$ such that $\emptyset \neq \omega(x) \subset K$ and thus $K$ cannot be negatively stable, therefore II does not hold; III implies the existence of a $y \in M \backslash K$ such that $\emptyset \neq \alpha(y) \subset K$ which contradicts the stability of $K$; IV contradicts the existence of neighbourhood $U$ in Lemma 1. Analogously if $K$ is a repeller then none of conditions I, III and IV holds. Suppose $K$ is neither an attractor nor a repeller. If III does not hold, then

a) $K$ is non-stagnant

or

b) for every $W \in \mathscr{N}_{K}, \quad \operatorname{Min}(W \backslash K) \neq \emptyset$

If b) holds then condition IV is clearly satisfied. Suppose a) holds. Since $K$ is neither an attractor nor a repeller, by lemma 1 , given any compact $V \in \mathscr{N}_{K}$, there are $x, y \in V \backslash K$ such that $\mathscr{O}^{-}(x) \subset V$ and $\mathscr{O}^{+}(y) \subset V$ (which implies $\emptyset \neq \alpha(x) \subset V$ and $\emptyset \neq \omega(y) \subset V)$. Since $K$ is non-stagnant we cannot have both $\alpha(x) \subset K$ and $\omega(y) \subset K$. If $\alpha(x) \not \subset K$ then there is a $z \in \alpha(x) \cap(V \backslash K)$ and

$$
\mathscr{O}(z) \subset \alpha(x) \subset V
$$

As $M \backslash K$ is invariant and $z \in M \backslash K, \mathscr{O}(z) \subset V \backslash K$. Since every neighbourhood of $K$ contains a compact $V \in \mathscr{N}_{K}$, condition IV necessarily holds. If $\omega(y) \not \subset K$ a time-symmetric argument conducts to the same conclusion.

The next result is, in a certain sense, a counterpart to the Butler-Mcgehee Lemma (Butler and Waltman [3], p.259).

Lemma 3. Let $M$ be a locally compact metric space with a $C^{0}$ flow $\theta$ and $X$ a nonvoid, compact, invariant proper subset of $M$. If $X$ is non-stagnant and $z \in A^{+}(X) \quad$ (resp. $\left.z \in A^{-}(X)\right)$ then given any $U \in \mathscr{N}_{K}$ there is a $y \in \omega(z)$ (resp. $y \in \alpha(z))$ such that $\mathscr{O}(y) \subset U \backslash X$. 
Proof. The result will be proved in the case $z \in A^{+}(X)$, a time symmetric argument disposes of case $z \in A^{-}(X)$. Assume the hypothesis of Lemma 3 hold. Given any $U \in \mathscr{N}_{K}$, let $0<\lambda<\min \left(1,|\omega(z)|_{X}\right)$ be such that $V:=$ $B[X, \lambda] \subset U$ is compact. We will first show that there are $p, q \in \omega(z)$ with $\mathscr{O}^{-}(p)$ and $\mathscr{O}^{+}(q)$ both nonvoid and contained in $V \backslash X$. Since $\omega(z) \cap X \neq$ $\emptyset$ and $\omega(z) \not \subset V$ it is easily seen that there are sequences $t_{n}, T_{n} \in \mathbb{R}^{+}$such that:

1) $t_{n}<T_{n}<t_{n+1}$ for all $n \geq 1$

2) $t_{n} \longrightarrow+\infty$

3) $\operatorname{dist}\left(z_{t_{n}}, K\right) \longrightarrow 0$

4) $a_{n}:=z_{T_{n}} \in \operatorname{bd} V$

5) $\theta\left(\left[t_{n}, T_{n}\right] \times\{z\}\right) \subset V$ which is equivalent to $\theta\left(\left[-\left(T_{n}-t_{n}\right), 0\right] \times\left\{a_{n}\right\}\right) \subset V$

Let $\left(a_{n_{i}}\right)$ be a subsequence of $\left(a_{n}\right)$ converging to some point $p$ of the compact bd $V$. Note that $a \in \omega(z)$ since $T_{n_{i}} \longrightarrow+\infty$. We claim that $\mathscr{O}^{-}(p) \subset V \backslash X$ : $\mathscr{O}(p) \subset M \backslash X$ since $p$ belongs to the invariant set $M \backslash X$. To prove $\mathscr{O}^{-}(p) \subset V$ observe that together, $X$ is a compact invariant set, $a_{n_{i}} \in$ bd $V \subset M \backslash X$, bd $V$ is compact, $z_{t_{n_{i}}}=\theta\left(-\left(T_{n_{i}}-t_{n_{i}}\right), a_{n_{i}}\right)$ and $\operatorname{dist}\left(z_{t_{n_{i}}}, X\right) \longrightarrow 0$ imply, by an argument identical to the one given in the proof of the Lemma 1, that we must have $-\left(T_{n_{i}}-t_{n_{i}}\right) \longrightarrow-\infty$. Now together,

$$
\begin{gathered}
\theta\left(\left[-\left(T_{n_{i}}-t_{n_{i}}\right), 0\right] \times\left\{a_{n_{i}}\right\}\right) \subset V, \\
a_{n_{i}} \longrightarrow p \text { and }-\left(T_{n_{i}}-t_{n_{i}}\right) \longrightarrow-\infty
\end{gathered}
$$

imply in virtue of the continuity of the flow (again as in Lemma 1), that $\mathscr{O}^{-}(p) \subset V$ and therefore $\operatorname{cl} \mathscr{O}^{-}(p)=\mathscr{O}^{-}(p) \cup \alpha(p) \subset V$. A similar argument ${ }^{23}$ (see fig.12) shows the existence of a $q \in \omega(z)$ such that $\mathscr{O}^{+}(q) \subset V \backslash X$, which by its turn implies $\operatorname{cl} \mathscr{O}^{+}(q)=\mathscr{O}^{+}(q) \cup \omega(q) \subset V$. Note that both $\alpha(p) \neq \emptyset$ and $\omega(q) \neq \emptyset$, as $V$ is a nonvoid compact. Now since $X$ is non-stagnant and $p, q \in M \backslash X$, we cannot have both $\emptyset \neq \alpha(p) \subset X$ and $\emptyset \neq \omega(q) \subset X$, therefore as $M \backslash X$ is invariant, there is necessarily a $y \in \alpha(p)$ such that $\mathscr{O}(y) \subset V \backslash X$ or there is a $y \in \omega(q)$ such that $\mathscr{O}(y) \subset V \backslash X$. In both cases $\mathscr{O}(y) \subset \omega(z)$ since $p, q \in \omega(z)$ and $\omega(z)$ is closed and invariant. As $V \backslash X \subset U \backslash X$ the proof is complete.

\footnotetext{
${ }^{23}$ observe that there are sequences $t_{n}, T_{n} \in \mathbb{R}^{+}$satisfying conditions 1) to 4) above plus
}

$\left.5^{\prime}\right) \quad \theta\left(\left[T_{n}, t_{n+1}\right] \times\{z\}\right) \subset V$ which is equivalent to $\theta\left(\left[0,\left(t_{n+1}-T_{n}\right)\right] \times\left\{a_{n}\right\}\right) \subset V$ 
Definition. Let $M$ be a metric space with a $C^{0}$ flow. A sequence $A_{n} \subset M$ approaches $X \subset M$ if for every $\varepsilon>0, A_{n} \subset B(X, \varepsilon)$ for all sufficiently large $n$ i.e. if $\left|A_{n}\right|_{X} \longrightarrow 0$.

In the next proof we use the following elementary result:

"In a metric space a sequence converges to a point $z$ if every subsequence contains a (sub)subsequence converging to $z$ ”.

Lemma 4. Let $M$ be a locally compact metric space with a $C^{0}$ flow. If a sequence $\Lambda_{n} \in \mathrm{Ci}(M)$ approaches a compact minimal set $S$ then $\left(\Lambda_{n}\right)$ actually $d_{H}$-converges to $S$ i.e.

$$
\left|\Lambda_{n}\right|_{S} \longrightarrow 0 \Longrightarrow \Lambda_{n} \stackrel{d_{H}}{\longrightarrow} S
$$

Proof. Let $U$ be a compact neighbourhood of $K$. Since $\left|\Lambda_{n}\right|_{S} \longrightarrow 0$, given any subsequence $\left(\Lambda_{n_{i}}\right)$, there is a $i_{0} \geq 1$ such that $\Lambda_{n_{i}} \subset U$ for all $i>i_{0}$. Now $\left[\mathrm{Ci}(U), d_{H}\right]$ is a compact metric space by Blaschke Theorem (see section 2), hence by Blaschke Principle there is a (sub)subsequence $\left(\Lambda_{n_{i_{k}}}\right) d_{H}$-converging to some nonvoid, compact, invariant set $Q \subset U$. But $\left|\Lambda_{n_{i_{k}}}\right|_{S} \longrightarrow 0$ implies $Q \subset S$ and since $S$ is a minimal set, $Q=S$. Hence the above convergence criterion is satisfied.

Therefore, $X \in \mathrm{CMin}(M)$ is an isolated minimal set ${ }^{24}$ iff $X$ is $d_{H}$-isolated in $\operatorname{CMin}(M)$ (lemma 4 establishes $(\Longleftarrow) ;(\Longrightarrow)$ follows from the definition of the Hausdorff metric).

Lemma 5. Let $M$ be a locally compact metric space with a $C^{0}$ flow. If $Q \in \mathrm{CMin}(M)$ then for each $\varepsilon>0$ there is a $\delta>0$ such that

$$
\operatorname{Ci}(B(Q, \delta)) \subset B_{H}(Q, \varepsilon) .
$$

Proof. Suppose the contrary. Then there is an $\varepsilon>0$ and sequences $\lambda_{n}>0$ and $\Lambda_{n} \in \operatorname{Ci}\left(B\left(Q, \lambda_{n}\right)\right)$ such that $\lambda_{n} \longrightarrow 0$ and $d_{H}\left(\Lambda_{n}, Q\right) \geq \varepsilon$. But this contradicts Lemma 4 since $\Lambda_{n} \in \operatorname{Ci}(M), Q \in \operatorname{CMin}(M)$ and $\left|\Lambda_{n}\right|_{Q} \longrightarrow 0$.

The above useful relation between the metric $d$ of $M$ and the Hausdorff metric $d_{H}$ of $\mathrm{C}(M)$ will be repeatedly used. Recall that if $A \subset M$ and $\mathfrak{M} \subset 2^{M}$ then $\mathfrak{M}(A)$ is the set of all $X \in \mathfrak{M}$ contained in $A$ (see section 2). Note lemma 5 and Hausdorff metric's definition together imply that if $\mathfrak{M} \subset \mathrm{CMin}(M)$, then for every $X \in \mathfrak{M}$ and $\varepsilon>0$, there is a $\delta>0$ such that

$$
B_{H}(X, \delta) \cap \mathfrak{M} \subset \mathfrak{M}(B(X, \delta)) \subset B_{H}(X, \varepsilon) \cap \mathfrak{M}
$$

\footnotetext{
${ }^{24}$ recall (section 2), that $X \in \mathrm{CMin}(M)$ is an isolated minimal (set) if there is a neighbourhood $U \subset M$ of $X$ such that $U \backslash X$ contains no minimal set of the flow.
} 
hence there are arbitrarily small $d_{H}$-neighbourhoods of $X \in \mathfrak{M}$ in the form $\mathfrak{M}(B(X, \delta))$, a most useful fact.

Lemma 6. Let $M$ be a locally compact metric space with a $C^{0}$ flow. If $\mathfrak{M} \subset \mathrm{CMin}(M)$ and for every $X \in \mathfrak{M}, \varepsilon>0$,

$$
\# \mathfrak{M}(B(X, \varepsilon))=\mathfrak{c} \quad(\text { resp. } \quad \mathfrak{M}(B(X, \varepsilon) \backslash X) \neq \emptyset)
$$

then $\mathfrak{M}$ is $\mathfrak{c}$-dense in itself (resp. $\mathfrak{M}$ is $d_{H}$-dense in itself).

Hence a set of compact minimal sets $\mathfrak{M}$ is $\mathfrak{c}$-dense in itself (resp. $d_{H}$-dense in itself) iff every neighbourhood $U_{X} \subset M$ of each $X \in \mathfrak{M}$ contains a continuum of elements of $\mathfrak{M}$ (resp. an element of $\mathfrak{M}$ distinct from $X$ ).

Proof of lemma 6. 1) Suppose that for every $X \in \mathfrak{M}$ and $\varepsilon>0, \# \mathfrak{M}(B(X, \lambda))=$ c. Let $\varepsilon>0$ and $X \in \mathfrak{M}$ be given. Since $\mathfrak{M} \subset \operatorname{CMin}(M) \subset \operatorname{Ci}(M)$, by lemma 5, for $\delta>0$ sufficiently small,

$$
\mathfrak{M}(B(X, \delta)) \subset \operatorname{Ci}(B(X, \delta)) \subset B_{H}(X, \varepsilon)
$$

therefore $\#\left(B_{H}(X, \varepsilon) \cap \mathfrak{M}\right)=\mathfrak{c} \quad$ since by hypothesis $\# \mathfrak{M}(B(X, \delta))=\mathfrak{c}$. Hence $\mathfrak{M}$ is $\mathfrak{c}$-dense in itself. 2) Suppose now that for every $X \in \mathfrak{M}$ and $\varepsilon>0, \quad \mathfrak{M}(B(X, \varepsilon) \backslash X) \neq \emptyset$. Then, given any $X \in \mathfrak{M}$, there is a sequence $\Lambda_{n} \in \mathfrak{M} \backslash\{X\} \subset \operatorname{Ci}(M)$ such that $\left|\Lambda_{n}\right|_{X} \longrightarrow 0$ and since $X \in \mathrm{CMin}(M)$, by lemma 4 it follows that $\Lambda_{n} \stackrel{d_{H}}{\longrightarrow} X$. As all the $\Lambda_{n}^{\prime} s$ are distinct from $X, X$ is not $d_{H}$-isolated in $\mathfrak{M}$, hence as $X \in \mathfrak{M}$ is arbitrary, $\mathfrak{M}$ is $d_{H}$-dense in itself.

Lemma 7. Let $M$ be a locally compact metric space with a $C^{0}$ flow. If $A \subset M$ is open and $\mathrm{CMin}(A)$ is $d_{H}$-dense in itself then $\mathrm{CMin}(A)$ is $\mathfrak{c}$-dense in itself. If $\mathfrak{M}$ is a $d_{H}$-open and dense in itself subset of $\mathrm{CMin}(M)$ then $\mathfrak{M}$ is $\mathfrak{c}$-dense in itself

The proof of lemma 7 is presented on section 8 .

Lemma 8. Let $M$ be a metric space. If $\mathfrak{C} \subset \mathrm{C}(M)$ and $A \subset M$ is open, then $\mathfrak{C}(A):=\{X \in \mathfrak{C}: X \subset A\}$ is $d_{H}$-open in $\mathfrak{C}$.

Proof. Let $X \in \mathfrak{C}(A)$. Since $X$ is compact and $A$ is open, there is a $\lambda>0$ such that $B(X, \lambda) \subset A$. On the other hand for any $\varepsilon>0, Y \in B_{H}(X, \varepsilon) \Longrightarrow$ $Y \subset B(X, \varepsilon)$. Therefore $Y \in \mathfrak{C} \cap B_{H}(X, \varepsilon) \Longrightarrow Y \in \mathfrak{C}(B(X, \varepsilon)) \Longrightarrow Y \in$ $\mathfrak{C}(A)$.

Lemma 9. Let $M$ be a metric space with a $C^{0}$ flow and $x_{n}$ a sequence of periodic points with (minimal) period $\lambda_{n}$. If $x_{n} \longrightarrow x$ and $\lambda_{n}$ is convergent then $x$ is either a periodic point or an equilibrium. 
Proof. Assume $x$ is neither a periodic point nor an equilibrium. Suppose $\lambda_{n} \longrightarrow T \in\left[0,+\infty\left[\right.\right.$. Let $d:=\operatorname{dist}\left(x, x_{T}\right) / 3>0$. By the continuity of the flow, there is a $0<\delta<d$ such that

$$
(z \in B(x, \delta) \text { and } t \in] T-\delta, T+\delta[) \Longrightarrow \theta(t, z) \in B\left(x_{T}, d\right)
$$

Since $x_{n} \longrightarrow x$ and $\lambda_{n} \longrightarrow T$, there is a $n_{0} \in \mathbb{N}$ such that

$$
n>n_{0} \Longrightarrow\left(x_{n} \in B(x, \delta) \subset B(x, d) \text { and } \lambda_{n} \in\right] T-\delta, T+\delta[)
$$

and therefore, as $x_{n}$ is periodic with period $\lambda_{n}$, it follows that for $n>n_{0}$, $x_{n}=\theta\left(\lambda_{n}, x_{n}\right) \in B\left(x_{T}, d\right)$ and $x_{n} \in B(x, d)$, which is absurd since $B(x, d) \cap$ $B\left(x_{T}, d\right)=\emptyset$. This actually shows that $x$ is either an equilibrium or $x$ is a periodic point with period $T / n$, for some $n \in \mathbb{N}$.

Lemma 10. Let $M$ be a locally compact metric space with a $C^{0}$ flow. If $Q$ is a compact aperiodic minimal set, then for any $m \geq 1$ there is an $\varepsilon>0$ such that

$$
\gamma \in \operatorname{Per}(M) \text { and } \operatorname{dist}(\gamma, Q)<\varepsilon \Longrightarrow \operatorname{period}(\gamma)>m
$$

Proof. Suppose the contrary. Then there is a $m \geq 1$ and there are sequences $\gamma_{n} \in \operatorname{Per}(M)$ and $x_{n} \in \gamma_{n}$ such that $\operatorname{dist}\left(x_{n}, Q\right) \longrightarrow 0$ and $0<\operatorname{period}\left(x_{n}\right)=$ $\operatorname{period}\left(\gamma_{n}\right) \leq m$. Since $Q$ has a compact neighbourhood of the form $B[Q, \delta]$, for some $\delta>0$, it is easily seen (taking $K_{n}:=B[Q, \delta / n]$ ) that applying Lemma 11.b) followed by Bolzano-Weierstrass Theorem, we may select from $\left(x_{n}\right)$ a sub-sequence $\left(x_{n_{i}}\right)$ such that $x_{n_{i}} \longrightarrow x \in Q$ and $\operatorname{period}\left(x_{n_{i}}\right) \longrightarrow$ $\lambda \in[0, m]$. By lemma 9 this implies $x \in Q$ is either a periodic point or an equilibrium, which is absurd since $Q \in \operatorname{Am}(M)$.

Lemma 11(Nested Compacts Lemma). Let $M$ be a locally compact metric space and $\mathfrak{C}$ a $d_{H}$-closed subset of $\mathrm{C}(M)$. If $K_{n} \in \mathfrak{C}$ and $K_{n} \supset K_{n+1}$ for all $n \geq 1$ then:

A) $K_{n} \stackrel{d_{H}}{\longrightarrow}\left(\bigcap_{n \geq 1} K_{n}\right) \in \mathfrak{C}$,

B) every sequence $x_{n} \in K_{n}$ has a subsequence converging to some $x \in \bigcap_{n \geq 1} K_{n}$.

C) every sequence $\Lambda_{n} \subset K_{n}$ where $\Lambda_{n} \in \mathfrak{B}, \mathfrak{B}$ a $d_{H}$-closed subset of $\mathrm{C}(M)$, has a subsequence $d_{H}$-converging to some $X \in \mathfrak{B}\left(\bigcap_{n \geq 1} K_{n}\right)$.

Proof. A) By Blaschske Theorem, $\left[\mathrm{C}\left(K_{1}\right), d_{H}\right]$ is a compact metric space (see section 2) and in addition, $K_{n} \in \mathrm{C}\left(K_{1}\right) \subset \mathrm{C}(M)$ for all $n \geq 1$, hence by Blaschke Principle there is a sub-sequence $\left(K_{n_{i}}\right)$ converging to some $K \in \mathrm{C}\left(K_{1}\right) \cap \mathfrak{C}$. 
This actually implies 25 the whole sequence $\left(K_{n}\right) d_{H}$-converges to $K$, since $K_{n} \supset K_{n+1}$ for all $n \geq 1$. Now $\bigcap_{n \geq 1} K_{n} \subset K$ since for each $z \in \bigcap_{n \geq 1} K_{n}$, the sequence $x_{n}:=z \in K_{n}$ converges to $z$, which implies $z \in \lim K_{n}=K$. On the other hand, $K_{m}$ is compact for each $m \geq 1$ and $K_{m} \supset K_{m+k}$ for all $k \geq 1$, hence since each $\mathrm{C}\left(K_{m}\right)$ is $d_{H}$-closed in $\mathrm{C}\left(K_{1}\right)$ (by Blaschke Theorem) it follows that $\lim K_{n}=K \subset K_{m}$ for each $m \geq 1$, therefore $K \subset \bigcap_{n \geq 1} K_{n}$ and finally $K=\bigcap_{n \geq 1} K_{n}$. B) Select from $x_{n} \in K_{n} \subset K_{1}$ a subsequence $\left(x_{n_{i}}\right)$ converging to some $x \in K_{1}$ (this is possible since $K_{1}$ is compact). Then $x_{n_{i}} \in K_{n_{i}}$ and $K_{n_{i}} \stackrel{d_{H}}{\longrightarrow} \bigcap_{n \geq 1} K_{n}$, thus necessarily $x \in \bigcap_{n \geq 1} K_{n}$. C) By Blaschke Principle, $\Lambda_{n} \in \mathfrak{B}\left(K_{n}\right) \subset \mathfrak{B}\left(K_{1}\right) \subset \mathrm{C}\left(K_{1}\right)$ has a subsequence $\left(\Lambda_{n_{i}}\right) d_{H}$-converging to some $X \in \mathrm{C}\left(K_{1}\right) \cap \mathfrak{B}$. But $\Lambda_{n_{i}} \subset K_{n_{i}} \stackrel{d_{H}}{\longrightarrow} \bigcap_{n \geq 1} K_{n}$ implies $\Lambda_{n_{i}}$ approaches $K=\bigcap_{n \geq 1} K_{n}$ i.e. $\left|\Lambda_{n_{i}}\right|_{K} \longrightarrow 0$, thus necessarily $X \subset \bigcap_{n \geq 1} K_{n}$ and finally $X \in \mathfrak{B}\left(\bigcap_{n \geq 1} K_{n}\right)$.

Lemma 12(Cantor-Dirichlet Principle). If $A=\bigcup_{1 \leq n \leq n_{0}} A_{n}$ and $A$ is infinite then $\# A_{n}=\# A$ for some $1 \leq n \leq n_{0}$.

Proof. Recall that if $D=B \cup C$, and $D$ is infinite then $B$ or $C$ is infinite (otherwise $\# D \leq \# B+\# C<\infty$ ), and also that using the Axiom of Choice, if $B$ and $C$ are two sets, at least one of which is infinite, then $\#(B \cup C)=$ $\max \{\# B, \# C\}$. Therefore by finite induction over $1 \leq n \leq n_{0}$ it follows that $\# A=\max \left\{\# A_{n}: 1 \leq n \leq n_{0}\right\}$.

\subsection{THE MAIN THEOREM. TOPOLOGICAL STRUCTURE OF CMin $(M)$.}

Again let $M$ be a locally compact, connected metric space with a $C^{0}$ flow. Consider the following six propositions where the variable $X$ assumes values in the set $\mathrm{Ci}(M)$ of nonvoid, compact, invariant subsets of $M$ :

1.X $X$ is an attractor.

2.X $X$ is a repeller.

3.X $X$ is isolated from minimals and stagnant.

4.X $X$ is isolated from minimals and there is a $X$ - $\alpha$ shell.

5.X $\quad X$ is isolated from minimals and there is a $X$ - $\omega$ shell.

6.X $X$ is isolated from minimals and there is a $X$-tree.

${ }^{25}$ using the elementary fact that if $A, B, C, Y \in \mathrm{C}(M), A \supset B \supset C, d_{H}(A, Y)<\varepsilon$ and $d_{H}(C, Y)<\varepsilon$ then $d_{H}(B, Y)<\varepsilon$. 
Theorem 1. Let $M$ be a locally compact, connected metric space with a $C^{0}$ flow $\theta$ and $K$ a compact, invariant proper subset of $M$. Then:

either

1. $K$ is an attractor.

or

2. $K$ is a repeller.

or at least one of the following four conditions holds:

3. $K$ is isolated from minimals and stagnant.

4. $K$ is isolated from minimals and there is a $K$ - $\alpha$ shell.

5. $K$ is isolated from minimals and there is a $K$ - $\omega$ shell.

6. $K$ is isolated from minimals and there is a $K$-tree.

or at least one of the following eighteen conditions hold

7.i $1 \leq i \leq 6$

there is a sequence $\left\{e_{n}\right\} \in \mathrm{Eq}(M \backslash K), d_{H}$-converging to some $\{q\} \in \mathrm{Eq}(\mathrm{bd} K)$ and such that condition $i . X$ is satisfied by all equilibrium orbits $\left\{e_{n}\right\}$.

8.i $1 \leq i \leq 6$

there is a sequence $\gamma_{n} \in \operatorname{Per}(M \backslash K), d_{H}$-converging to some $Q \in \mathfrak{S}(\mathrm{bd} K)$ and such that condition $i . X$ is satisfied by all periodic orbits $\gamma_{n}$.

9.i $1 \leq i \leq 6$

there is a sequence $\Gamma_{n} \in \operatorname{Am}(M \backslash K), d_{H}$-converging to some $Q \in \mathfrak{S}(\mathrm{bd} K)$ and such that condition i.X is satisfied by all compact aperiodic minimals $\Gamma_{n}$. or

10. there is an open neighbourhood $U$ of $K$ such that $\operatorname{CMin}(U \backslash K)$ is $\mathfrak{c}$-dense in itself and at least one of the following four conditions holds:

10.1 $\mathrm{Eq}(U \backslash K)$ is a $\mathfrak{c}$-dense in itself set, $d_{H}$-accumulating in $\mathrm{Eq}(\mathrm{bd} K)$.

10.2 $\operatorname{Per}(U \backslash K)$ is a $\mathfrak{c}$-dense in itself set, $d_{H}$-accumulating in $\mathfrak{S}(\mathrm{bd} K)$.

10.3 $\operatorname{Am}(U \backslash K)$ is a $\mathfrak{c}$-dense in itself set, $d_{H}$-accumulating in $\mathfrak{S}(\mathrm{bd} K)$.

10.4 there are $\mathfrak{c}$-dense in itself sets $P \subset \operatorname{Per}(U \backslash K)$ and $A \subset \operatorname{Am}(U \backslash K)$, $d_{H}$-open in $\operatorname{Per}(M)$ and in $\operatorname{Am}(M)$, respectively, and such that:

- both $P$ and $A d_{H}$-accumulate in $\mathfrak{S}(\mathrm{bd} K)$

- $K$ is bi-stable in relation to $P^{*}=\bigcup_{\gamma \in P} \gamma$ and $A^{*}=\bigcup_{\Gamma \in A} \Gamma$

- for any sequence $\gamma_{n} \in P$, $\operatorname{dist}\left(\gamma_{n}, K\right) \rightarrow 0$ implies period $\left(\gamma_{n}\right) \rightarrow+\infty$. 
7.4

K

7.5

K

7.6
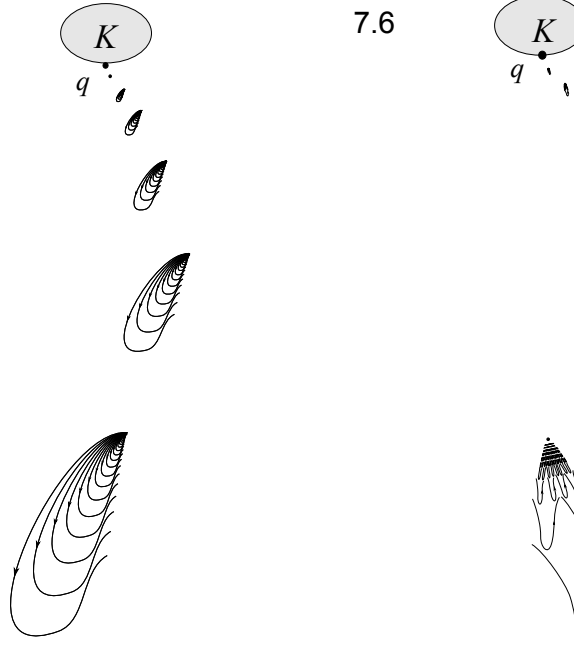

Fig. 13:

8.4

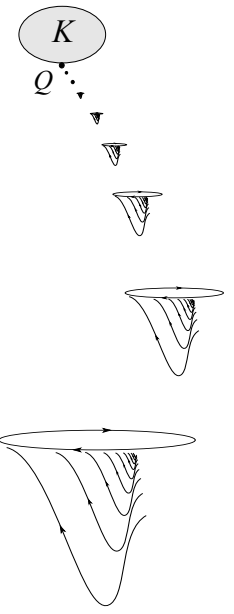

8.5

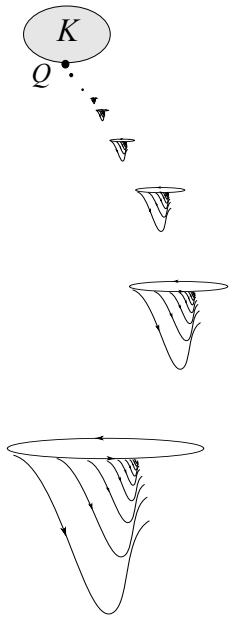

Fig. 14:
8.6

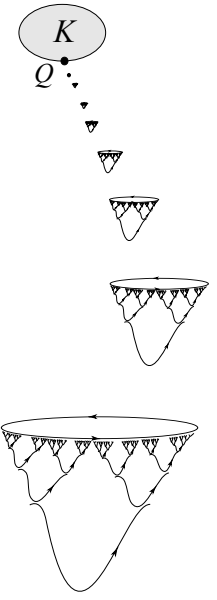

Observe that, $\gamma_{n} \in P$ and dist $\left(\gamma_{n}, K\right) \rightarrow 0$ together actually imply that $\left|\gamma_{n}\right|_{K} \rightarrow 0$, since $P^{*}$ is bi-stable in relation to $K$. On the other hand, by the 1 st point of 10.4 there is a sequence $P \ni \gamma_{n} \stackrel{d_{H}}{\longrightarrow} Q \in \mathfrak{S}(\mathrm{bd} K)$. As $P$ is $\mathfrak{c}$-dense in itself, the 3 rd point can thus be replaced by the following condition:

- given any $n \geq 1$, all periodic orbits $\gamma \in P$ contained in a sufficiently small neighbourhood $V$ of $K$ have period $>n$, and there is always a continuum of these.

Remark. Due to the fact that, for any given $\varepsilon>0$, a $X$-tree always has a sub $X$-tree with all its orbits contained in $B(X, \varepsilon) \backslash X$, and analogue fact holds for 


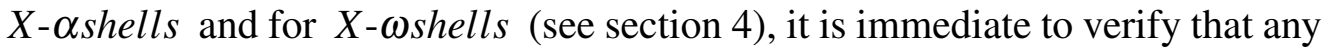
of the twelve conditions $4,5,6,7.4$ to $7.6,8.4$ to 8.6 and 9.4 to 9.6 implies that for every neighbourhood of $U$ of $K$, there is a sequence of orbits $\gamma_{n} \subset U \backslash K$ such that

$$
\operatorname{cl} \gamma_{1} \supsetneq \operatorname{cl} \gamma_{2} \supsetneq \cdots \cdots \supsetneq \operatorname{cl} \gamma_{n} \supsetneq \cdots \cdots
$$

Thus the claim of condition $\mathbf{H}$ in section 3 is justified.

9.4

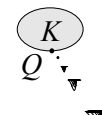

$T$

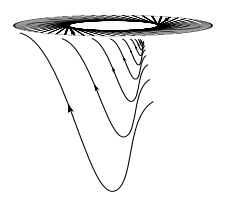

9.5
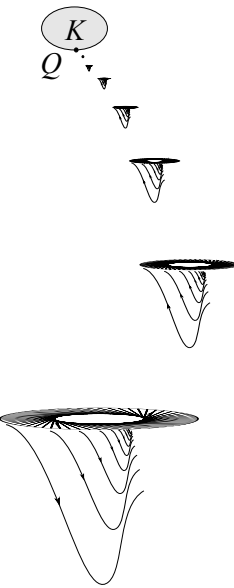

9.6

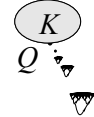

Fig. 15: examples 9.4 to 9.6 show sequences of 2-tori carrying irrational linear subflows.

INTERLUDE: TOPOLOGICAL STRUCTURE OF CMin $(M)$.

Theorem 1 brings to light the importance of compact minimal sets to the characterization of the possible "dynamical landscapes" in the vicinity of a compact invariant proper subset of a flow. Obviously there is a close relation between the dynamical behaviour of a flow near a compact minimal set $X$ and the topological Hausdorff structure of $\operatorname{CMin}(M)$ near $X$. Actually, from Theorem 1 we easily obtain an elegant characterization of the set $\mathrm{CMin}(M)$ of all compact minimal sets of the flow, endowed with the Hausdorff metric.

Let $M$ be a locally compact, connected metric space with a $C^{0}$ flow. Consider the following seven propositions, where the variable $X$ now takes values in the set $\mathrm{CMin}(M)$ of all compact minimal subsets of the flow:

1.X $\quad X$ is an attractor.

2.X $\quad X$ is a repeller.

3.X $\quad X$ is an isolated minimal set and stagnant.

4.X $\quad X$ is an isolated minimal set and there is a $X-\alpha$ shell. 
5.X $\quad X$ is an isolated minimal set and there is a $X$ - $\omega$ shell.

6.X $X$ is an isolated minimal set and there is a $X$-tree.

10.X there is an $\varepsilon>0$ such that the compact minimal sets contained in $B(X, \varepsilon)$ form a $\mathfrak{c}$-dense in itself subset of $\mathrm{CMin}(M)$.

or equivalently (by lemma 6 , section 5 ),

$10^{\prime} . X$ there is an $\varepsilon>0$ such that every neighbourhood $U_{Y} \subset M$ of each $Y \in \mathrm{CMin}(B(X, \varepsilon))$ contains a continuum of compact minimal sets.

If $X$ satisfies $10 . X$, then as $X$ is itself a compact minimal set, every neighbourhood of $X$ actually contains a continuum of compact minimal sets.

Important remark: Recall that, the definition of the Hausdorff metric and lemma 4 (section 5), together imply that

" $X$ is an isolated compact minimal set iff it is $d_{H}$-isolated in $\mathrm{CMin}(M) . "$

(by definition (section 2), $X$ is an isolated minimal (set) if for some $U \in \mathscr{N}_{X}$, $U \backslash X$ contains no minimal set of the flow).

Definition. denote by $\mathfrak{M}_{i}, 1 \leq i \leq 6$ or $i=10$ the set of all $X \in \mathrm{CMin}(M)$ satisfying condition $i . X$ and by $\mathfrak{M}_{i-j}, \quad 1 \leq i<j \leq 6$ the set of all $X \in$ $\mathrm{CMin}(M)$ satisfying (at least) one of condition i.X to $j . X$.

Theorem 2. Let $M$ be a locally compact, connected metric space with a $C^{0}$ flow. Then:

1) $\mathfrak{M}_{1-6}$ is the set of isolated compact minimal sets and thus a countable, $d_{H}$-open subset of $\mathrm{CMin}(M)$.

2) $\mathfrak{M}_{10}$ is a $d_{H}$-open and $\mathfrak{c}$-dense in itself subset of $\mathrm{CMin}(M)$. It is either empty or has the cardinal of the continuum.

3) $\mathfrak{M}_{1-6}$ is $d_{H}$-dense in $\operatorname{CMin}(M) \backslash \mathfrak{M}_{10}$.

Proof. In first place, note the following trivial fact that will be implicitly used in several instances bellow: if $X$ is a compact minimal set and $Y_{n}$ is a sequence of compact minimal sets $d_{H}$-converging to $Q \in \mathfrak{S}(X)$ then $Q=X$.

1) Clearly every $X \in \mathfrak{M}_{1-6}$ is an isolated compact minimal set; on the other hand, by Theorem 1, any compact minimal set $X$ satisfying none of the six conditions $1 . X$ to $6 . X$ is not an isolated compact minimal set. ${ }^{26}$ hence $\mathfrak{M}_{1-6}$ is the set of isolated compact minimal sets of the flow. By a remark above, every $X \in \mathfrak{M}_{1-6}$ is thus $d_{H}$-isolated in $\operatorname{CMin}(M)$, hence $\mathfrak{M}_{1-6}$ is $d_{H}$-open

\footnotetext{
${ }^{26}$ note that if $X \in \operatorname{CMin}(M)$ satisfies none of conditions $1 . X$ to $6 . X$, then $X$ is necessarily a proper subset of $M$, as $M$ compact implies $M$ is both an attractor and a repeller in the flow, hence Theorem 1 can be applied.
} 
in $\operatorname{CMin}(M) . \quad \mathfrak{M}_{1-6}$ is countable since it is a $d_{H}$-discrete and separable metric space. ${ }^{27}$

2) If $X \in \mathfrak{M}_{10}$ then there is an $\varepsilon_{X}>0$ such that $\operatorname{CMin}\left(B\left(X, \varepsilon_{X}\right)\right)$ is a $\mathfrak{c}$-dense in itself, $d_{H}-$ open subset of $\operatorname{CMin}(M)$ (lemma 8 , section 5). It is immediate to verify that $\operatorname{CMin}\left(B\left(X, \varepsilon_{X}\right)\right) \subset \mathfrak{M}_{10}$ : if $Y \in \operatorname{CMin}\left(B\left(X, \varepsilon_{X}\right)\right)$ then $Y \subset$ $B\left(X, \varepsilon_{X}\right)$ and since $Y$ is compact, $\varepsilon_{X}-|Y|_{X}>0$, thus $B\left(Y, \varepsilon_{X}-|Y|_{X}\right) \subset$ $B\left(X, \varepsilon_{X}\right)$, hence as $B\left(Y, \varepsilon_{X}-|Y|_{X}\right)$ is open in $M$, by lemma $8, \operatorname{CMin}\left(B\left(Y, \varepsilon_{X}-\right.\right.$ $\left.\left.|Y|_{X}\right)\right)$ is a $d_{H}$-open subset of $\operatorname{CMin}\left(B\left(X, \varepsilon_{X}\right)\right)$ and hence $\mathfrak{c}$-dense in itself. Thus $Y \in \mathfrak{M}_{10}$ and therefore $\mathfrak{M}_{10}=\bigcup_{X \in \mathfrak{M}_{10}} \operatorname{CMin}\left(B\left(X, \varepsilon_{X}\right)\right)$ is a $\mathfrak{c}$-dense in itself, $d_{H}$-open subset of $\mathrm{CMin}(M)$. Since the phase space $M$ is separable, there is at most a continuum of compact minimal sets in the flow, therefore $\mathfrak{M}_{10}$ is either empty or has cardinal $\mathfrak{c}$, since it is $\mathfrak{c}$-dense in itself.

3) We claim that $\mathfrak{M}_{10}$ is the set of compact minimal sets of the flow satisfying condition 10 of Theorem 1 (taking $K:=X$ ): if $X \in \mathfrak{M}_{10}$ then it clearly satisfies none of the 24 conditions 1 to 9.6 (since $X$ is not the $d_{H}$-limit of a sequence of isolated compact minimal sets), hence by the same theorem, it must satisfy condition 10. On the other hand, if $X$ satisfies condition 10, then there is an open $U \in \mathscr{N}_{X}$ such that $\operatorname{CMin}(U \backslash X)$ is $\mathfrak{c}$-dense in itself, hence taking $\varepsilon>0$ such that $B(X, \varepsilon) \subset U$, it follows (by lemma 8) that $\operatorname{CMin}(B(X, \varepsilon) \backslash X)$ is a $d_{H}$-open subset of $\operatorname{CMin}(U \backslash X)$ and hence $\mathfrak{c}$-dense in itself. Also by Theorem 1, at least one of the 4 conditions $\mathbf{1 0 . 1}$ to $\mathbf{1 0 . 4}$ is satisfied, hence $X$ is the $d_{H}$-limit of a sequence of compact minimal sets $Y_{n} \in$ $\operatorname{CMin}(B(X, \varepsilon) \backslash X)$. As $\operatorname{CMin}(B(X, \varepsilon) \backslash X)$ is $\mathfrak{c}$-dense in itself, this implies that in every neighbourhood of $X$ there is a continuum of compact minimal sets contained in $B(X, \varepsilon)$. Hence $\operatorname{CMin}(B(X, \varepsilon))$ is $\mathfrak{c}$-dense in itself, thus $X$ satisfies condition $10 . X$. Therefore, by Theorem 1 , if $X \in \operatorname{CMin}(M) \backslash \mathfrak{M}_{10}$ then $X$ must satisfy (at least) one of the 24 conditions 1 to 9.6 and this clearly implies $X \in \mathrm{cl}_{H} \mathfrak{M}_{1-6}$ since $\mathfrak{M}_{i}, 1 \leq i \leq 6$ is the set of compact minimal sets of the flow satisfying condition $1 \leq \mathbf{i} \leq 6$ of Theorem 1 .

Note, however, that $10 . X$ is indeed a very strong condition, essentially due to its $d_{H}$-openness: even when $\mathrm{CMin}(M) \backslash \mathfrak{M}_{1-6}$ is nonvoid and $\mathfrak{c}$-dense in itself, it can happen that $\mathfrak{M}_{7}$ is empty, since it is still possible that $\mathfrak{M}_{1-6}$ is $d_{H}$-dense in the whole $\operatorname{CMin}(M)$ (simple examples of $C^{\infty}$ flows exhibiting this phenomenon already occur on $\mathbb{S}^{1}$ and $\mathbb{S}^{2}$ ). However, the next result shows that a nonvoid $\mathfrak{c}$-dense in itself set of compact minimal sets always occurs, whenever there are uncountably many compact minimal sets in the flow. More precisely, if $\mathrm{CMin}(M)$ is uncountable, then removing from this set a suitable countable (possibly empty) set we obtain a nonvoid $\mathfrak{c}$-dense in itself set of compact minimal sets. This decomposition theorem is analogous to the

\footnotetext{
${ }^{27}$ recall (section 8.2) that $\mathrm{C}(M) \supset \mathfrak{M}_{1-6}$ is $d_{H}$-separable.
} 
celebrated Cantor-Bendixson Theorem for separable, complete metric spaces (Polish spaces). Note, however, that although $d_{H}$-separable ${ }^{28}$ in general, $\operatorname{CMin}(M)$ is neither $d_{H}$-complete nor $d_{H}$-locally compact. Also observe that, since there is at most a continuum of compact minimal sets in the flow (see section 2), the above result implies that $\operatorname{CMin}(M)$ obeys, in a certain sense, to the Continuum Hypothesis: its cardinal is either finite (possibly null), denumerable $\left(\boldsymbol{\aleph}_{0}\right)$ or the continuum $\mathfrak{c}=2 \aleph_{0}$.

Theorem 3. Let $\theta$ be a $C^{0}$ flow on a locally compact, separable metric space $M$, displaying uncountably many compact minimal sets. Then there is a countable (possibly empty) set $\mathfrak{I} \subset \mathrm{CMin}(M)$ such that:

I. $\mathfrak{D}:=\operatorname{CMin}(M) \backslash \mathfrak{I}$ is a $\mathfrak{c}$-dense in itself and $d_{H}$-closed subset of $\mathrm{CMin}(M)$, having the cardinal of the continuum.

II. $\mathfrak{I}$ is the set of all $X \in \mathrm{CMin}(M)$ having a neighbourhood containing only countably many (possibly one) compact minimal sets, hence $\mathfrak{D}$ is the largest $\mathfrak{c}$-dense in itself subset of $\mathrm{CMin}(M)$.

Hence, if $\operatorname{CMin}(M)$ is uncountable, then all but a countable number of compact minimal sets of the flow have a continuum of compact minimal sets on each of their neighbourhoods or, equivalently, $\operatorname{CMin}(M)$ is the union of a countable (possibly empty) set and $a \mathfrak{c}$-dense in itself set.

The proof uses in an essential way a "Cantor's ternary set - like" construction that constitutes the core of the proof of lemma 7 (section 8.1).

Proof. Suppose $\mathrm{CMin}(M)$ is uncountable. Let $\mathfrak{I}$ be the set of all $X \in$ $\operatorname{CMin}(M)$ for which there is an $\varepsilon>0$ such that

$B_{H}(X, \varepsilon)$ contains only countably many (possibly only one) compact minimals.

(by lemma 5 this is actually equivalent to: there is a $\delta>0$ such that there are only countably many compact minimal sets contained in $B(X, \delta) \subset M)$. For each $X \in \mathfrak{I}$ define

$$
\varepsilon_{X}:=\sup \left\{\varepsilon>0: B_{H}(X, \varepsilon) \cap \operatorname{CMin}(M) \text { is countable }\right\}
$$

Note that $0<\varepsilon_{X}<+\infty$ since $\operatorname{CMin}(M)$ is, by hypothesis, uncountable and $\operatorname{CMin}(M)=\bigcup_{n \geq 1}\left(B_{H}(X, n) \cap \operatorname{CMin}(M)\right)$. Also, observe that for each $X \in \mathfrak{I}$, $B_{H}\left(X, \varepsilon_{X}\right) \cap \mathrm{CMin}(M)$ is a countable $d_{H}$-open subset of $\operatorname{CMin}(M)$ and thus is contained in $\mathfrak{I}$ (by definition of $\varepsilon_{X}, B_{H}\left(X, \varepsilon_{X}(1-1 / n)\right) \cap \mathrm{CMin}(M)$ is countable for each $n \in \mathbb{N}$, hence $B_{H}\left(X, \varepsilon_{X}\right) \cap \mathrm{CMin}(M)=\bigcup_{n \geq 1}\left(B_{H}(X,(1-\right.$ $\left.\left.1 / n) \varepsilon_{X}\right) \cap \operatorname{CMin}(M)\right)$ is countable).

\footnotetext{
28 since $\mathrm{C}(M) \supset \mathrm{CMin}(M)$ is, see section 8.2.
} 
Claim 1: $\mathfrak{I}$ is countable: assume $\mathfrak{I}$ is infinite (otherwise the claim is proved). $\mathrm{CMin}(M)$ is $d_{H}$-separable (since $\mathrm{C}(M) \supset \mathrm{CMin}(M)$ is), ${ }^{29}$ hence $\mathfrak{I}$ is $d_{H}$-separable. Let $I=\left\{X_{1}, X_{2}, \ldots, X_{n}, \ldots\right\}$ be a denumerable $d_{H}$-dense subset of $\mathfrak{I}$. We claim that

$$
\mathfrak{I}=\bigcup_{n \geq 1}\left(B_{H}\left(X_{n}, \varepsilon_{X_{n}}\right) \cap \mathrm{CMin}(M)\right)
$$

therefore proving the countability of $\mathfrak{I}$. The inclusion $\supset$ is already established by a remark above. To prove $\subset$ observe that given any $Y \in \mathfrak{I}$, there is a $X_{n} \in I$ such that $X_{n} \in B_{H}\left(Y, \varepsilon_{Y} / 2\right)$. Thus, by the triangle inequality for the $d_{H}$ metric, $B_{H}\left(X_{n}, \varepsilon_{Y} / 2\right) \subset B_{H}\left(Y, \varepsilon_{Y}\right)$, hence $B_{H}\left(X_{n}, \varepsilon_{Y} / 2\right) \cap \operatorname{CMin}(M) \subset$ $B_{H}\left(Y, \varepsilon_{Y}\right) \cap \operatorname{CMin}(M)$ is countable. Therefore $\varepsilon_{X_{n}} \geq \varepsilon_{Y} / 2$. Hence $Y \in$ $B_{H}\left(X_{n}, \varepsilon_{X_{n}}\right) \cap \mathrm{CMin}(M)$ since $d_{H}\left(X_{n}, Y\right)<\varepsilon_{Y} / 2$. The claim is proved. Note that the identity above also proves that $\mathfrak{I}$ is $d_{H}$-open in $\operatorname{CMin}(M)$.

Now let $\mathfrak{D}:=\operatorname{CMin}(M) \backslash \mathfrak{I}$. Clearly $\mathfrak{D}$ is nonvoid since $\operatorname{CMin}(M)$ is uncountable (by hypothesis) and $\mathfrak{I}$ is countable. Actually, by definition of $\mathfrak{I}$, given any $X \in \mathfrak{D}$ and $\varepsilon>0, B_{H}(X, \varepsilon) \cap \operatorname{CMin}(M)$ is uncountable, hence $B_{H}(X, \varepsilon) \cap \mathfrak{D}$ is also uncountable and in particular, $\mathfrak{D}$ is a nonvoid $d_{H}$-dense in itself subset of $\mathrm{CMin}(M)$.

Claim 2: $\mathfrak{D}$ is $\mathfrak{c}$-dense in itself: As $\mathfrak{D} \subset \operatorname{CMin}(M)$, in virtue of lemma 6 (section 5), we need only to prove that given any $X \in \mathfrak{D}$ and $\varepsilon>0$, there is a continuum of compact minimal sets $Y \in \mathfrak{D}$ contained in $B(X, \varepsilon) \subset M$. Taking $\varepsilon$ sufficiently small we may assume $B[X, \varepsilon]$ is compact ( $X$ is compact and $M$ is locally compact). Let $A:=B(X, \varepsilon), \Lambda_{0}:=X$ and $\varepsilon_{0}:=\varepsilon / 2$. Now since $\mathfrak{D}$ is $d_{H}$-dense in itself, we may carry the construction of the proof of lemma 7 (section 8.1) within $\mathfrak{D}(A)=\{Z \in \mathfrak{D}: Z \subset A\}$ i.e. we may select each $\Lambda_{a}, a \in \mathscr{F}$ in $\mathfrak{D}(A)$ instead of in $\operatorname{CMin}(A)$. As in the proof of lemma 7 we get a continuum of $d_{H}$-Cauchy sequences, $d_{H}$-converging to a continuum of mutually disjoint, nonvoid, compact invariant sets contained in $A$, therefore proving the existence of a continuum of compact minimal sets contained in in this open set (as each $K \in \mathrm{Ci}(A)$ contains at least one compact minimal set). Now since $\mathfrak{I}$ is countable, a continuum of these compact minimal sets $\Gamma \in \operatorname{CMin}(A)$ actually belongs to $\mathfrak{D}=\operatorname{CMin}(M) \backslash \mathfrak{I}$. Therefore $\mathfrak{D}$ is $\mathfrak{c}$-dense in itself. $\mathfrak{D}$ is $d_{H}$-closed in $\operatorname{CMin}(M)$ since $\mathfrak{I}$ is $d_{H}$-open in the same set.

It is simple to see that the set $E$ of equilibria satisfies the following stronger analogue property to that expressed on Theorem 3:

" if $E$ is uncountable, then $E$ is the union of a countable set and a perfect subset $\mathfrak{E}$ of $M$, with the cardinal of the continuum. For each $z \in \mathfrak{E}$ and

\footnotetext{
${ }^{29}$ see section 8.2
} 
$\varepsilon>0$ there is an embedding $h$ of Cantor's ternary set into $B(z, \varepsilon) \cap \mathfrak{E}$ with $z \in \operatorname{im} h$. Hence $\mathfrak{E} \subset E$ is a $\mathfrak{c}$-dense in itself closed subset of $M$.

The question now arises as whether the corresponding propositions analogue to Theorem 3, for the set $\operatorname{Per}(M)$ of all periodic orbits and for the set $\operatorname{Am}(M)$ of all compact aperiodic minimal sets of the flow, also hold

I. If $\operatorname{Per}(M)$ is uncountable then all but a countable number of periodic orbits of the flow have a continuum of periodic orbits on each of their neighbourhoods.

II. If $\operatorname{Am}(M)$ is uncountable then all but a countable number of compact aperiodic minimal sets of the flow have a continuum of compact aperiodic minimal sets on each of their neighbourhoods.

As already mentioned in the introduction, it is, in a certain sense 31 useless to look for counterexamples to any of these two propositions within "standard" Dynamical Systems Theory: both I. and II. are provable in ZFC set theory under the additional assumption of the Continuum Hypothesis $\mathrm{CH}$. Hence each turns out to be either demonstrable in $Z F C$ or independent of this standard axiomatic (due to Gödel's result, 1938). The proof that $C H \Longrightarrow \mathrm{I} \wedge \mathrm{II}$ is simple and actually depends only on the fact $\operatorname{Per}(M)$ is a separable (Hausdorff) metric space: I. and II. are particular cases of the following proposition, which is equivalent to the Continuum Hypothesis:

$\mathfrak{c}-$ Denseness Hypothesis $(\mathfrak{c} D H):$ If $L$ is an uncountable separable metric space, then a $\mathfrak{c}$-dense in itself set is obtained removing from $L$ a suitable countable set (possibly empty). 32

As we could locate no reference for the equivalence $\mathrm{CH} \Longleftrightarrow \mathfrak{c D H}$, a short proof is included in section 8.3, for the sake of completeness.

PROOF OF THEOREM 1.

Synopsis: Assume neither $\mathbf{1}$ nor $\mathbf{2}$ hold. A) If $K$ is isolated from minimal sets then it is shown that at least one of conditions $\mathbf{3}$ to $\mathbf{6}$ necessarily holds. B) If $K$ is not isolated from minimals, then we consider two possible cases: B.1) if for every neighbourhood $U$ of $K, U \backslash K$ contains a compact minimal set of $(M, \theta)$

\footnotetext{
30 This follows immediately from the following observation: since the phase space $M$ is locally compact and separable it can be endowed with an equivalent boundedly compact metric (on which every closed bounded set is compact, see e.g. [9], p.278), thus becoming a complete, separable metric space (Polish space). $E$ is closed in $M$, hence is also a Polish space in this equivalent metric, and this proposition is well known to hold on such spaces (see e.g. [8], chap. VII.2).

31 i.e. working within Zermelo-Fraenkel Set Theory and provided this "standard" axiomatic is consistent.

${ }^{32}$ here $X \subset M$ is $\mathfrak{c}$-dense in itself means, as for the $d_{H}$ metric, that for every $x \in X$ and $\varepsilon>0, B(x, \varepsilon) \cap X$ has the cardinal $\mathfrak{c}$ of the continuum.
} 
satisfying (at least) one of the six conditions $1 . X$ to $6 . X$, then it is proved that at least one of the eighteen cases 7.1 to 9.6 holds; B.2) if the contrary is true, then there is a neighbourhood $U$ of $K$ such that $\operatorname{CMin}(U \backslash K)$ is a $\mathfrak{c}$-dense in itself, $d_{H}$-open subset of $\operatorname{CMin}(M), d_{H}$-accumulating in $\mathfrak{S}(K)$ and at least one of the four conditions $\mathbf{1 0 . 1}$ to $\mathbf{1 0 . 4}$ necessarily holds.

Proof of Theorem 1. It easily seen that condition $\mathbf{1}$ excludes the remaining 27 conditions and the same holds with condition 233 Assume, throughout the remaining of this proof, that neither $\mathbf{1}$ nor $\mathbf{2}$ holds. In this situation we distinguish the two possible cases:
A) $K$ is isolated from minimals i.e. for some $U \in \mathscr{N}_{K}, \quad \operatorname{CMin}(U \backslash K)=\emptyset$.
B) for every $V \in \mathscr{N}_{K}, \operatorname{CMin}(V \backslash K) \neq \emptyset$.

We recall an important elementary that will be implicitly used in several instances bellow: on a locally compact metric space, every sufficiently small neighbourhood of a compact set has compact closure, and thus may only contain compact minimal sets.

Case A):

Since $K$ is compact and $M$ is locally compact. we may assume, without loss of generality, that $U$ is compact. Then for any $z \in U$,

$$
\begin{aligned}
& \mathscr{O}^{+}(z) \subset U \Longrightarrow \omega(z) \cap K \neq \emptyset \Longrightarrow z \in A^{+}(K) \sqcup B^{+}(K) \\
& \mathscr{O}^{-}(z) \subset U \Longrightarrow \alpha(z) \cap K \neq \emptyset \Longrightarrow z \in A^{-}(K) \sqcup B^{-}(K)
\end{aligned}
$$

since otherwise we would have $\operatorname{CMin}(U \backslash K) \neq \emptyset \quad$ (clearly $\mathscr{O}^{+}(z) \subset U \Longrightarrow$ $\operatorname{cl} \mathscr{O}^{+}(z)=\mathscr{O}^{+}(z) \cup \omega(z) \subset U$, thus if $\omega(z) \cap K=\emptyset$ then $\omega(z) \subset U \backslash K$. But $\omega(z)$ is a nonvoid, compact, invariant set (since $\mathscr{O}^{+}(z) \subset U$ is compact, hence it must contain a compact minimal set of $(M, \theta)$, in contradiction with $\operatorname{CMin}(U \backslash K)=\emptyset$. If $\mathscr{O}^{-}(z) \subset U$, then assuming $\alpha(z) \cap K=\emptyset$ we arrive at the same contradiction). Suppose now that condition $\mathbf{3}$ does not hold. Since $K$ is isolated from minimals it follows that $K$ is non-stagnant, therefore for every orbit $\mathscr{O}(z) \subset U \backslash K$,

$$
\begin{array}{lll}
\text { either } & 0 . & z \in A^{-}(K) \cap B^{+}(K) \\
\text { or } & \text { I. } & z \in B^{-}(K) \cap A^{+}(K) \\
\text { or } & \text { II. } & z \in A^{-}(K) \cap A^{+}(K)
\end{array}
$$

\footnotetext{
${ }^{33}$ Actually, if $K$ is an attractor then $B^{+}(K) \backslash K \neq \emptyset$ and $x \in B^{+}(K) \backslash K \Longrightarrow \alpha(x) \subset$ bd $B^{+}(K) \subset M \backslash B^{+}(K)$ (see footnotes 13 and 25). A time-symmetric fact holds if $K$ is a repeller. This immediately implies that if $\mathbf{1}$ (resp. 2) holds, then none of the remaining 27 conditions can take place.
} 
Depending on which of these three cases is satisfied, we say $\mathscr{O}(z)$ is an orbit of type 0, I or II. More generally, the fact $K$ is non-stagnant implies that orbits of type 0 and I cannot coexist in $U \backslash K$. This implies that exactly one of the following three conditions holds:

i) there is an orbit $\mathscr{O}(x) \subset U \backslash K$ such that $(\operatorname{cl} \mathscr{O}(x)) \backslash K$ contains only orbits of type 0

ii) there is an orbit $\mathscr{O}(y) \subset U \backslash K$ such that $(\operatorname{cl} \mathscr{O}(y)) \backslash K$ contains only orbits of type I

iii) for every orbit $\mathscr{O}(z) \subset U \backslash K, \quad(\operatorname{cl} \mathscr{O}(z)) \backslash K$ contains an orbit of type II (observe that $\mathscr{O}(w) \subset U \backslash K \Longrightarrow(\operatorname{cl} \mathscr{O}(w)) \backslash K \subset U \backslash K)$. We claim that

a) implies there is a $K$ - $\alpha$ shell

b) ii) implies there is a $K$ - $\omega$ shell

c) iii) implies there is a $K$-tree

Suppose there is an orbit $\mathscr{O}(y)$ satisfying condition ii). Since $\mathscr{O}(y)$ is of type I, by Lemma 3 (recall that $K$ is, by hypothesis, non-stagnant), given any neighbourhood $V$ of $K$, there is a $p \in \omega(y) \backslash K \subset \operatorname{cl} \mathscr{O}(y)$ such that $\mathscr{O}(p) \subset$ $V \backslash K$. Clearly $\mathscr{O}(p)$ is also of type I since $\mathscr{O}(p) \subset \operatorname{cl} \mathscr{O}(y)$. The existence of a $K$ - $\omega$ shell with first orbit $\mathscr{O}(y)$ is now a straightforward inductive consequence of Lemma 3. Analogously, if $\mathscr{O}(x)$ is an orbit satisfying condition i) then there is a $K$ - $\alpha$ shell with first orbit $\mathscr{O}(x)$. We now prove c). Recall that by hypothesis, $K$ satisfies none of conditions 1, 2 and 3, therefore by Lemma 2 , there is necessarily an orbit $\mathscr{O}(z) \subset U \backslash K$. By iii), $(\operatorname{cl} \mathscr{O}(z)) \backslash K$ contains an orbit $\gamma_{0}$ of type II. We will inductively define a map

$$
\begin{aligned}
\psi: \mathscr{E} & \longrightarrow \operatorname{Orb}\left(\left(\mathrm{cl} \gamma_{0}\right) \backslash K\right) \subset \operatorname{Orb}(U \backslash K) \subset \operatorname{Orb}(M \backslash K) \\
a & \longmapsto \gamma_{a}
\end{aligned}
$$

so that $\Theta:=\operatorname{im} \psi$ is a $K$-tree. Adopt the following lexicographic order on $\mathscr{E}$ :

$$
0<00<01<000<001<010<011<0000<0001<0010<\cdots \ldots
$$

Suppose $a \in \mathscr{E}$ is such that for all $\mathscr{E} \ni d<a, \gamma_{d}$ is an already defined orbit of type II contained in $\left(\mathrm{cl} \gamma_{0}\right) \backslash K \subset U \backslash K$. We define $\gamma_{a}$ :

Evidently, $a=b c$ for some $b \in \mathscr{E}$ and $c \in\{0,1\}$. By Lemma $3{ }^{34}$ there is an orbit $\zeta_{b c}$ such that:

- $\gamma_{b} \succ^{c} \zeta_{b c}$

\footnotetext{
${ }^{34}$ note that $K \in \operatorname{Ci}(M) \backslash\{M\}, K$ is non-stagnant and by hypothesis, $\gamma_{b}$ is of type II i.e. $\gamma_{b} \subset A^{-}(K) \cap A^{+}(K)$.
} 
- $0<\left|\zeta_{b c}\right|_{K}<\left|\gamma_{b}\right|_{K} / 2$

hence $\zeta_{b c} \nsucc \gamma_{b}$ and $\zeta_{b c} \subset\left(\operatorname{cl} \gamma_{b}\right) \backslash K \subset\left(\operatorname{cl} \gamma_{0}\right) \backslash K \subset U \backslash K$. By hypothesis iii), $\left(\mathrm{cl} \zeta_{b c}\right) \backslash K$ contains an orbit of type II and we identify $\gamma_{a}$ with it. Clearly $\gamma_{b} \succ^{c} \gamma_{b c}=\gamma_{a}$ for every $b \in \mathscr{E}, c \in\{0,1\}$ since $\gamma_{a} \subset \mathrm{cl} \zeta_{b c}$ and $\gamma_{b} \succ^{c} \zeta_{b c}$. Note that inequality $\left|\gamma_{a}\right|_{K}=\left|\gamma_{b c}\right|_{K} \leq\left|\zeta_{b c}\right|_{K}<\left|\gamma_{b}\right| / 2$ guarantees $\gamma_{b c} \nsucc \gamma_{b}$ for every $b \in \mathscr{E}, c \in\{0,1\}$ and $\left|\operatorname{cl} \gamma_{v_{n}}\right|_{K} \longrightarrow 0$ for every $v \in \mathrm{E}_{\infty}$. It is now immediate to verify that $(\Theta, \psi)$, where $\Theta$ is the inductively defined set $\left\{\gamma_{a}: a \in \mathscr{E}\right\}$, is indeed a $K$-tree.

We thus conclude that case A) implies that at least one of conditions $\mathbf{3}, \mathbf{4}, \mathbf{5}$ or 6 necessarily holds, therefore if conditions 1 to 6 (i.e. $1 . K$ to $6 . K$ ) all fail then condition B) holds (recall we assumed, in the beginning of the proof, that both $\mathbf{1}$ and $\mathbf{2}$ are false). Note that since $K$ is an arbitrary compact, invariant, proper subset of $M$, the above observation is true for all $X \in \operatorname{Ci}(M) \backslash\{M\}$ i.e.

Lemma 13. If $X$ is a compact, invariant, proper subset of $M$ and all conditions $1 . X$ to $6 . X$ fail, then arbitrarily near $X$ there is always a compact minimal set of $(M, \theta)$ disjoint from $X$ i.e. for any $\varepsilon>0, \operatorname{CMin}(B(X, \varepsilon) \backslash X) \neq \emptyset$.

Case B): for every $V \in \mathscr{N}_{K}, \quad \operatorname{CMin}(V \backslash K) \neq \emptyset$.

We distinguish the two (sub)cases:

B.1) for every $V \in \mathscr{N}_{K}, \quad V \backslash K$ contains a compact minimal set $X$ satisfying (at least) one of the six conditions $1 . X$ to 6.X.

B.2) there is an open $U \in \mathscr{N}_{K}$ such that no $X \in \operatorname{CMin}(U \backslash K)$ satisfies any of the six conditions $1 . X$ to $6 . X$.

Case B.1):

We will show that in this case at least one of the eighteen cases 7.1 to 9.6 will necessarily hold. Take $\varepsilon_{1}>0$ such that $U:=B\left[K, \varepsilon_{1}\right]$ is compact. Let $\Lambda_{1}$ be a compact minimal set contained in $U \backslash K$, satisfying at least one of the six conditions $1 . X$ to $6 . X$. Since $K$ and $\Lambda_{1}$ are disjoint compacts, $B\left(K, \varepsilon_{2}\right) \cap$ $\Lambda_{1}=\emptyset$ where $\varepsilon_{2}:=\operatorname{dist}\left(\Lambda_{1}, K\right) / 2$. We may obviously define two sequences $\Lambda_{n} \in \mathrm{CMin}(U \backslash K)$ and $\varepsilon_{n}>0$ such that for every $n \geq 1$,

- $\Lambda_{n}$ satisfies at least one of the six conditions $1 . X$ to $6 . X$

- $\Lambda_{n} \subset B\left[K, \varepsilon_{n}\right]$

- $\varepsilon_{n+1}:=\operatorname{dist}\left(\Lambda_{n}, K\right) / 2$

Since $\Lambda_{n} \in \operatorname{CMin}(U) \subset \mathfrak{S}(U)$ for all $n \geq 1$ and $\left|\Lambda_{n}\right|_{K} \longrightarrow 0$, by Lemma 11 , we may select from $\left(\Lambda_{n}\right)$ a subsequence $d_{H}$-converging to some $Q \in$ 
$\mathfrak{S}(K) \cdot{ }^{35}$ Obviously $Q \subset$ bd $K$, since $\Lambda_{n} \subset M \backslash K$, thus in fact $Q \in \mathfrak{S}($ bd $K)$. By the Cantor-Dirichlet Principle we may select from this subsequence another subsequence consisting of compact minimal sets all belonging to the same one of the following three classes: equilibrium orbits $\operatorname{Eq}(M)$, periodic orbits $\operatorname{Per}(M)$, compact aperiodic minimals $\operatorname{Am}(M)$. Finally since each term of $\left(\Lambda_{n}\right)$ satisfies at least one of the six conditions 1.X to 6.X, using the Cantor-Dirichlet Principle again, we select from the last obtained subsequence another subsequence such that (at least) one of the six conditions 1.X to 6.X is satisfied by all its terms, therefore obtaining a sequence of compact minimal sets contained in $\quad M \backslash K$ satisfying at least one of the eighteen conditions 7.1 to 9.6.

To complete the proof of Theorem 1 we show that in case B.2) i.e. if

a) $\operatorname{CMin}(V \backslash K) \neq \emptyset$ for all $V \in \mathscr{N}_{K}$

and

b) for some open $U \in \mathscr{N}_{K}$, no $X \in \operatorname{CMin}(U \backslash K)$ satisfies any of the six conditions $1 . X$ to $6 . X$

then at least one of the four cases $\mathbf{1 0 . 1}$ to $\mathbf{1 0 . 4}$ necessarily holds.

Case B.2):

We may obviously assume, without loss of generality, that $U$ has compact closure. Observe that:

- $\operatorname{CMin}(U \backslash K)$ is $d_{H}$-open in $\operatorname{CMin}(M)$ since $U \backslash K$ is open and $\operatorname{CMin}(M) \subset$ $\mathrm{C}(M)$ (Lemma 8).

- In virtue of Lemma 13, $\mathfrak{b})$ implies that for any $X \in \operatorname{CMin}(U \backslash K)$ and $\varepsilon>0, \quad \operatorname{CMin}(B(X, \varepsilon) \backslash X) \neq \emptyset$, thus by Lemma 5, every $X \in \operatorname{CMin}(U \backslash$ $K)$ is non $d_{H}$-isolated in $\operatorname{CMin}(M)$ and since $\operatorname{CMin}(U \backslash K)$ is $d_{H}$-open in $\operatorname{CMin}(M)$ (lemma 8), it follows that every $X \in \operatorname{CMin}(U \backslash K)$ is non $d_{H}$-isolated in $\operatorname{CMin}(U \backslash K)$ i.e. $\operatorname{CMin}(U \backslash K)$ is $d_{H}$-dense in itself. By Lemma 7, $\operatorname{CMin}(U \backslash K)$ is in fact $\mathfrak{c}$-dense in itself.

- Using Lemma 11 (Nested Compacts Lemma) $\sqrt{36}$ we infer from a) that $\operatorname{CMin}(U \backslash K) \quad d_{H}$-accumulates in $\mathfrak{S}(K)$.

Therefore, in case B.2), there is an open $U \in \mathscr{N}_{K}$ with compact closure such that

\footnotetext{
${ }^{35} \mathrm{n} \Lambda_{n} \in \mathfrak{S}(M), \quad \Lambda_{n} \subset B\left[K, \varepsilon_{n}\right] \in \mathrm{C}(M)$ and $\varepsilon_{n+1}<\varepsilon_{n} / 2$, thus by Lemma 11.c), $\Lambda_{n} \stackrel{d_{H}}{\longrightarrow}$ $Q \in \mathfrak{S}\left(\bigcap_{n \geq 1} B\left[K, \varepsilon_{n}\right]\right)=\mathfrak{S}(K)$.

${ }^{36}$ Let $n_{0} \geq 1$ be such that $B\left[K, 1 / 2^{n_{0}}\right] \subset U$ and define $K_{n}:=B\left[K, 1 / 2^{n}\right] \in \mathrm{C}(\mathrm{cl} U)$ for all $n \geq n_{0}$. Select a sequence $\Lambda_{n} \in \operatorname{CMin}\left(K_{n} \backslash K\right), n \geq n_{0}$. Note that $K_{n} \supset K_{n+1}, \bigcap_{n \geq n_{0}} K_{n}=K$ and all $\Lambda_{n}, n \geq n_{0}$ belong to the $d_{H}$-compact $\mathfrak{S}(\operatorname{cl} U)$, therefore $\left(\Lambda_{n}\right)$ has a subsequence $d_{H}$ - converging to some $Q \in \mathfrak{S}\left(\bigcap_{n \geq 1} K_{n}\right)=\mathfrak{S}(K)$.
} 
- $\operatorname{CMin}(U \backslash K)$ is a $\mathfrak{c}$-dense in itself, $d_{H}$-open subset of $\operatorname{CMin}(M), d_{H}-$ accumulating in $\mathfrak{S}(K)$.

Note that in particular, $\operatorname{Per}(U \backslash K) \sqcup \operatorname{Am}(U \backslash K)$ is a $\mathfrak{c}$-dense in itself, $d_{H}-$ open subset of $\mathrm{CMin}(M)$ since $\operatorname{Eq}(M)$ is a $d_{H}$-closed subset of $\operatorname{CMin}(M)$. Now the above remark concerning $\operatorname{CMin}(U \backslash K)$ implies there is a sequence $\Lambda_{n} \in \mathrm{CMin}(U \backslash K), d_{H}$-accumulating in $\mathfrak{S}(K)$. By the Cantor-Dirichlet Principle we may suppose this sequence is such that all $\Lambda_{n}$ belong to the same one of the following three classes: equilibrium orbits $\mathrm{Eq}(M)$, periodic orbits $\operatorname{Per}(M)$, compact aperiodic minimals $\operatorname{Am}(M)$.

Suppose now that conditions $\mathbf{1 0 . 1}, \mathbf{1 0 . 2}$ and $\mathbf{1 0 . 3}$ all fail. We will show that condition $\mathbf{1 0 . 4}$ is necessarily true. The equality $\operatorname{CMin}(N)=\mathrm{Eq}(N) \sqcup$ $\operatorname{Per}(N) \sqcup \operatorname{Am}(N)$, valid for all $N \subset M$, will be repeatedly used ( $\sqcup$ denotes disjoint union). Three possible cases are distinguished:

1st case: There is a sequence $\Lambda_{n} \in \operatorname{Am}(U \backslash K) \quad d_{H}$-accumulating in $\mathfrak{S}(K)$.

Since $\operatorname{Am}(U \backslash K) d_{H}$-accumulates in $\mathfrak{S}(K)$ but $\mathbf{1 0 . 3}$ is false, for every open $V \in \mathscr{N}_{K}$ there are $\Gamma \in \operatorname{Am}(V \backslash K)$ and $\varepsilon>0$ such that

$$
\#\left(B_{H}(\Gamma, \varepsilon) \cap \operatorname{Am}(V \backslash K)\right)<\mathfrak{c}
$$

Now since $V \backslash K$ is open and $\Gamma$ is closed, $B[\Gamma, \delta] \subset V \backslash K$ for a sufficiently small $\delta>0$. Moreover by Lemma $5(\Gamma \in \mathrm{CMin}(M)$ and $\operatorname{Am}(M) \subset \mathrm{Ci}(M))$, taking $\delta$ even smaller if necessary, we may further guarantee that

$$
\operatorname{Am}(B(\Gamma, \delta)) \subset B_{H}(\Gamma, \varepsilon) \cap \operatorname{Am}(V \backslash K)
$$

which implies

$$
\# \operatorname{Am}(B(\Gamma, \delta))<\mathfrak{c}
$$

Therefore it is easily seen that there are sequences $\Gamma_{n} \in \operatorname{Am}(U \backslash K)$ and $\delta_{n}>0$ such that:

1) $\left|\Gamma_{n}\right|_{K} \longrightarrow 0$

2) $B\left[\Gamma_{n}, \delta_{n}\right] \subset U \backslash K$

3) $\# \operatorname{Am}\left(B\left(\Gamma_{n}, \delta_{n}\right)\right)<\mathfrak{c}$

Again, by Lemma 11 (recall that $\Gamma_{n} \subset U \backslash K$ and $\operatorname{cl} U$ is compact) we may replace condition 1) above by

1) $\quad \Gamma_{n} \stackrel{d_{H}}{\longrightarrow} Q$ for some $Q \in \mathfrak{S}(\mathrm{bd} K)$

Clearly 
4) $\delta_{n}<d_{H}\left(\Gamma_{n}, Q\right)$

since $B\left[\Gamma_{n}, \delta_{n}\right] \cap Q=\emptyset$ (recall that $\emptyset \neq Q \subset K$ ).

Taking a smaller $\delta_{n}$ if necessary, we may further require that:

5) $\quad \mathrm{Eq}\left(B\left(\Gamma_{n}, \delta_{n}\right)\right)=\emptyset \quad 37$

6) $\gamma \in \operatorname{Per}\left(B\left(\Gamma_{n}, \delta_{n}\right)\right) \Longrightarrow \operatorname{period}(\gamma)>n$

$\operatorname{CMin}(U \backslash K)$ is nonvoid and $\mathfrak{c}$-dense in itself, thus so is $\operatorname{CMin}\left(B\left(\Gamma_{n}, \delta_{n}\right)\right)$ since $B\left(\Gamma_{n}, \delta_{n}\right) \subset U \backslash K$ is open and $\Gamma_{n} \in \operatorname{CMin}(M)$; also by 5$)$,

$$
\operatorname{CMin}\left(B\left(\Gamma_{n}, \delta_{n}\right)\right)=\operatorname{Per}\left(B\left(\Gamma_{n}, \delta_{n}\right)\right) \sqcup \operatorname{Am}\left(B\left(\Gamma_{n}, \delta_{n}\right)\right)
$$

hence in virtue of 3), the Cantor-Dirichlet Principle implies

7) $\left(X \in \operatorname{CMin}\left(\Gamma_{n}, \delta_{n}\right)\right.$ and $\left.\varepsilon>0\right) \Longrightarrow \# \operatorname{Per}(B(X, \varepsilon))=\mathfrak{c}$

In particular, $P_{n}:=\operatorname{Per}\left(B\left(\Gamma_{n}, \delta_{n}\right)\right)$ is an $d_{H}$-open, $\mathfrak{c}$-dense in itself subset of $\operatorname{Per}(M) d_{H}$-accumulating in $\Gamma_{n}$ (by 7 and Lemma 4). Let $P:=\bigcup_{n \in \mathbb{N}} P_{n}$. Then since $P d_{H}$-accumulates in $\Gamma_{n}$ and $\Gamma_{n} \stackrel{d_{H}}{\longrightarrow} Q \in \mathfrak{S}(\mathrm{bd} K)$, it follows that

- $P \subset \operatorname{Per}(M \backslash K)$ is a c-dense in itself, $d_{H}$-open subset of $\operatorname{Per}(M)$, $d_{H}$-accumulating in $\mathfrak{S}(\mathrm{bd} K)$.

Moreover,

- $K$ is bi-stable in relation to $P^{*}=\bigcup_{\gamma \in P} \gamma$ :

$P^{*}$ is a union of periodic orbits and hence invariant. Given any $V \in \mathscr{N}_{K}$ let $\lambda>0$ be such that $B(K, \lambda) \subset V$. Since $Q \subset K, d_{H}\left(\Gamma_{n}, Q\right) \longrightarrow 0$ and $\delta_{n}<d_{H}\left(\Gamma_{n}, Q\right)$, there is a $n_{0} \geq 1$ such that:

$$
\begin{gathered}
n>n_{0} \Longrightarrow d_{H}\left(\Gamma_{n}, Q\right)<\lambda / 2 \Longrightarrow \\
\Longrightarrow \Gamma_{n} \subset B(Q, \lambda / 2) \subset B(K, \lambda / 2) \text { and } \delta_{n}<\lambda / 2 \Longrightarrow \\
\Longrightarrow B\left(\Gamma_{n}, \delta_{n}\right) \subset B(K, \lambda) \Longrightarrow \\
\Longrightarrow P_{n}:=\operatorname{Per}\left(B\left(\Gamma_{n}, \delta_{n}\right)\right) \subset \operatorname{Per}(B(K, \lambda)) \subset \operatorname{Per}(V)
\end{gathered}
$$

Since $K$ is closed, by 2) there is a $0<\delta<\lambda / 2$ such that

$$
B(K, \delta) \bigcap\left(\bigcup_{1 \leq n \leq n_{0}} B\left[\Gamma_{n}, \delta_{n}\right]\right)=\varnothing
$$

\footnotetext{
${ }^{37}$ the set $\mathrm{Eq}(M) \subset \mathrm{C}(M)$ is $d_{H}$-closed and $\Gamma_{n} \notin \mathrm{Eq}(M)$, (see Lemma 4).

${ }^{38}$ By Lemma 10, this condition is necessarily satisfied for $\delta_{n}$ small enough since $\Gamma_{n} \in$ $\operatorname{Am}(M)$.
} 
Therefore,

$$
x \in B(K, \delta) \cap P^{*} \Longrightarrow\left(x \in P_{n}^{*} \text { for some } n>n_{0}\right) \Longrightarrow \mathscr{O}(x) \subset V
$$

because $\mathscr{O}(x) \in P_{n}$ and $P_{n} \subset \operatorname{Per}(V)$. The bi-stability of $K$ in relation to $P^{*}$ is proved.

- for any sequence $\gamma_{n} \in P, \operatorname{dist}\left(\gamma_{n}, K\right) \longrightarrow 0 \Longrightarrow \operatorname{period}\left(\gamma_{n}\right) \longrightarrow+\infty$ :

By 2), each $B\left[\Gamma_{n}, \delta_{n}\right]$ is a compact disjoint from $K$, hence given any $n_{0} \geq$ 1 , there is a $\delta>0$ satisfying the identity (8) above, therefore by 6),

$$
\begin{gathered}
(\gamma \in P \text { and } \gamma \cap B(K, \delta) \neq \emptyset) \Longrightarrow\left(\gamma \in P_{n} \text { for some } n>n_{0}\right) \Longrightarrow \\
\Longrightarrow \operatorname{period}(\gamma)>n>n_{0}
\end{gathered}
$$

2nd case: There is a sequence $\Lambda_{n} \in \operatorname{Per}(U \backslash K) d_{H}$-accumulating in $\mathfrak{S}(\mathrm{bd} K)$.

Since by hypothesis condition $\mathbf{1 0 . 2}$ is not true, an argument completely similar to that used in the 1 st case proves that

- there is a $\mathfrak{c}$-dense in itself set $A \subset \operatorname{Am}(M \backslash K), d_{H}$-open in $\operatorname{Am}(M)$, $d_{H}$-accumulating in $\mathfrak{S}(\mathrm{bd} K)$ and such that $K$ is bi-stable in relation to $A^{*}$.

3rd case: There is a sequence $\Lambda_{n} \in \mathrm{Eq}(U \backslash K) \quad d_{H}$-accumulating in $\mathrm{Eq}(\mathrm{bd} K)$.

Since by hypothesis $\mathbf{1 0 . 1}$ is not true, there are necessarily sequences $z_{n} \in$ $M$ and $\varepsilon_{n}>0$ such that:

$$
\left\{z_{n}\right\} \in \operatorname{Eq}(U \backslash K) \quad \text { and } \operatorname{dist}\left(z_{n}, K\right) \longrightarrow 0 \quad \text { and } \quad \# \mathrm{Eq}\left(B\left(z_{n}, \varepsilon_{n}\right)\right)<\mathfrak{c}
$$

By the $d_{H}$-closeness of $\operatorname{Eq}(M)$ in conjunction with Lemma 11, we may suppose that $\left\{z_{n}\right\} \stackrel{d_{H}}{\longrightarrow}\{z\}$ for some $\{z\} \in \operatorname{Eq}(K)$. Clearly $z \in$ bd $K$, thus $\{z\} \in$ $\mathrm{Eq}(\mathrm{bd} K)$. Now $C M i n(U \backslash K)$ is $\mathfrak{c}$-dense in itself, $\left\{z_{n}\right\} \in \operatorname{CMin}(U \backslash K)$ for all $n \geq 1, \operatorname{CMin}(U \backslash K)=\operatorname{Eq}(U \backslash K) \sqcup \operatorname{Per}(U \backslash K) \sqcup \operatorname{Am}(U \backslash K)$ and moreover $\# \mathrm{Eq}\left(B\left(z_{n}, \varepsilon_{n}\right)\right)<\mathfrak{c}$, therefore we infer using Cantor-Dirichlet Principle that there is a subsequence $\left(z_{n_{i}}\right)$ such that:

$\# \operatorname{Am}\left(B\left(z_{n_{i}}, \varepsilon\right)\right)=\mathfrak{c} \forall i \geq 1, \varepsilon>0$ or $\# \operatorname{Per}\left(B\left(z_{n_{i}}, \varepsilon\right)\right)=\mathfrak{c} \quad \forall i \geq 1, \varepsilon>0$

Thus by Lemma 4,

$$
\left\{z_{n_{i}}\right\} \in \mathrm{cl}_{H} \operatorname{Am}(U \backslash K) \quad \forall i \geq 1 \quad \text { or } \quad\left\{z_{n_{i}}\right\} \in \operatorname{cl}_{H} \operatorname{Per}(U \backslash K) \quad \forall i \geq 1
$$

and since $\left\{z_{n_{i}}\right\} \stackrel{d_{H}}{\longrightarrow}\{z\} \in \mathrm{Eq}(\mathrm{bd} K) \subset \mathfrak{S}(\mathrm{bd} K)$, it follows that there is a sequence in $\operatorname{Am}(U \backslash K) d_{H}$-accumulating in $\mathfrak{S}(\mathrm{bd} K)$ or there is a sequence 
in $\operatorname{Per}(U \backslash K) d_{H}$-accumulating in $\mathfrak{S}(\mathrm{bd} K)$. Thus, the 3 rd case implies the 1 st or the 2 nd. On the other hand, as we have seen, the 1st case clearly implies the 2 nd and vice versa, hence the 1 st and 2 nd cases always occur. Therefore if conditions 10.1, 10.2 and $\mathbf{1 0 . 3}$ are false then 10.4 is true. The proof of Theorem 1 is complete.

Q.E.D.

\section{INDEPENDENT REALIZATIONS. EXAMPLES.}

With Theorem 1 established, the question of whether all the 28 cases it describes are realizable naturally arises. Furthermore we may doubt whether all these cases are mutually independent. Let $(M, \theta)$ be a $C^{0}$ flow on a compact, connected metric space and $\Sigma$ one of the twenty eight conditions of Theorem 1. We say that $(M, \theta)$ is an independent realization of $\Sigma$ if there a compact, invariant, proper subset $K \subset M$ such that condition $\Sigma$ is satisfied for this choice of $M, K$ and $\theta$ but none of the remaining conditions of Theorem 1 holds, for the same $M, K$ and $\theta+39$

We can give the following answer to the questions raised above: all conditions, except the twelve involving $X$-trees, $X$ - $\alpha$ shells and $X$-wshells (that is, all but the "exceptional" conditions 4, 5, 6, 7.4 to 7.6, 8.4 to 8.6 and 9.4 to 9.6), admit independent realizations by $C^{\infty}$ flows on compact, connected manifolds (in fact on $\mathbb{S}^{n}$ for some $1 \leq n \leq 4$ ). Each of the exceptional conditions admits a $\left(C^{0}\right)$ independent realization on which $M$ is a compact, connected invariant subset of a $C^{\infty}$ flow $\phi$ on $\mathbb{R}^{n}, \theta$ is $\left.\phi\right|_{\mathbb{R} \times M}, K \subsetneq M$ is an equilibrium orbit of $\phi$ and $3 \leq n \leq 6$. A moment of reflexion shows that this implies that in the last sentence we may substitute $\mathbb{R}^{n}$ by any (2nd countable, Hausdorff) $C^{\infty}$ manifold $\mathscr{M}$ of dimension $m \geq n$. We call such an independent realization of one of the twelve exceptional conditions of Theorem 1 a subsmooth independent realization and call the manifold $\mathscr{M} \supset M$ carrying the $C^{\infty}$ flow $\phi$ the ambient manifold of this realization. Whether each of these twelve exceptional conditions admits an independent realization by a $C^{r}(r \geq$ 1) flow on a compact, connected $C^{\infty}$ manifold requires further exam. In [12] we give an independent realization for each one of the 28 conditions of Theorem 1 , in the line of what was stated above. Here, for the sake of brevity, we will confine our attention to the twelve exceptional conditions ${ }^{40}$ since these present greater difficulties than the others and display less known and more interesting dynamical phenomena. Actually the main difficulty lies in obtaining subsmooth independent realization of conditions $\mathbf{4}, 5$ and $\mathbf{6}$ with $K$ an equilibrium orbit, a periodic orbit and a compact aperiodic minimal. Once this done, we have the essential "dynamical pieces" for the construction of subsmooth independent

\footnotetext{
${ }^{39}$ Sometimes we actually identify a specific compact invariant proper subset $K$, saying that $(M, \theta), K$ constitutes an independent realization of the condition in question.

${ }^{40}$ i.e. to conditions $4,5,6,7.4$ to $7.6,8.4$ to $8.6,9.4$ to 9.6 .
} 
realizations of the remaining nine exceptional conditions $\mathbf{7 . 4}-\mathbf{7 . 6}, \mathbf{8 . 4}-\mathbf{8 . 6}$ and 9.4-9.6, which are then easily achieved through well known vector field constructions on trivial normal tubular neighbourhoods.

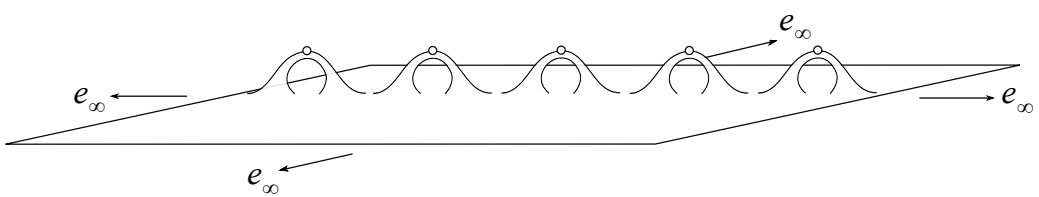

Fig. 16: The infinite genus smooth surface $S \subset \mathbb{R}^{3}$.

We shall first produce examples of subsmooth independent realizations for condition 6 with $K$, respectively, an equilibrium orbit, a periodic orbit and a compact aperiodic minimal (a $\mathbb{T}^{2}$ with an irrational linear flow) 4

Example 1. Subsmooth independent realization of condition 6 with $M$ an orbit closure of a $C^{\infty}$ flow $\zeta^{t}$ on $\mathbb{S}^{3} \subset \mathbb{R}^{4}, \theta=\left.\zeta^{t}\right|_{\mathbb{R} \times M}$ and $K=\{(0,0,0,1)\} \subsetneq M$ an equilibrium orbit.

Our point of departure is a beautiful example, due to Beniere and Meigniez [2], of a $C^{\infty}$ complete vector field $v$ generating a flow without minimal sets on a non-compact, orientable surface $\mathfrak{M}$ of infinite genus ${ }^{42}$ The set of end points $E(\mathfrak{M})$ is homeomorphic to $\Delta:=\{0\} \cup\{1 / n: n \in \mathbb{N}\} \subset \mathbb{R}$ and all end points are $f a t:{ }^{43}$ except the non-isolated one. We shall first construct a smoothly $\left(C^{\infty}\right)$ embedded surface $S \subset \mathbb{R}^{3}$ that is $C^{\infty}$ diffeomorphic to $\mathfrak{M}$. Operate the following $C^{\infty}$ surgery within the ambient manifold $\mathbb{R}^{3}$ : to the plane $\mathbb{R}^{2} \times\{0\} \subset \mathbb{R}^{3}$ smoothly add denumerably many handles 44 as shown in fig. 16. From each handle remove one point $e_{n}, n \in \mathbb{N}$. We obtain a non-closed, smooth 2-submanifold $S \subset \mathbb{R}^{3}$. As $\mathfrak{M}, S$ is an orientable $C^{\infty}$

\footnotetext{
${ }^{41}$ Remark. Vector fields on submanifolds $M \subset \mathbb{R}^{n}$ will be always represented in the usual abridged form $X: M \longrightarrow \mathbb{R}^{n}$ i.e. instead of considering $v: M \longrightarrow T M \subset T \mathbb{R}^{n}=\mathbb{R}^{n} \times \mathbb{R}^{n}$, we work with $X=\pi_{2} \circ v$, where $\pi_{2}$ is the projection onto the 2 nd factor. Where doubts may arise concerning what flow is aimed at, we indicate it inside brackets or as a subscript $e . g$. we write $\operatorname{Min}(X, \theta)$ and $\omega_{\theta}(z)$ instead of $\operatorname{Min}(X)$ and $\omega(z)$. When giving examples of flows generated by $C^{r} \quad(r \geq 1)$ vector fields on manifolds $M$, often the vector field in question is indicated as a subscript $e$.g. we write $\omega_{X}(z)$ for the $\omega$-limit set of $z$ on the flow $X^{t}$ generated by $X \in \mathfrak{X}^{r}(M)$. We use these notations freely (with the subscript indicating either the flow or the generating vector field) since no risk of ambiguity arises.

42 The first example of a $C^{\infty}$ flow on a manifold, without minimal sets was, to our knowledge, constructed on a surface by Takashi Inaba in 1995 (see [6]). This achievement is, in our opinion, one of the highlights of exceptional dynamics in the second half of the last century.

43 an end $e \in E(\mathfrak{M})$ is flat if it has a neighbourhood homeomorphic to $\mathbb{R}^{2}$ in the end-points compactification $\mathfrak{M}^{\propto}=\mathfrak{M} \sqcup E(\mathfrak{M})$ of $\mathfrak{M}$. Richards [10] calls such an end point "planar". Beniere and Meigniez [2] designate by $M$ our surface $\mathfrak{M}$.

44 the resulting surface is known as the infinite Loch-Ness monster.
} 
surface of infinite genus with all ends isolated and flat except one, $e_{\infty}$, which is both non-isolated and non-flat. This implies that its end points set $E(S)$ is also homeomorphic to $\Delta$ (see above), hence there is a homeomorphism $\xi: E(\mathfrak{M}) \longrightarrow E(S)$ sending the unique non-flat end of $\mathfrak{M}$ to the unique nonflat end of S. By Kerekjarto Theorem (see e.g. [10], p.262 and [2], p.26) the surfaces $\mathfrak{M}$ and $S$ are homeomorphic ${ }^{45}$ hence, as it is well known, $C^{\infty}$ diffeomorphic. Let $f: \mathfrak{M} \longrightarrow S$ be a $C^{\infty}$ diffeomorphism defining a $C^{\infty}$ embedding $\mathfrak{M} \hookrightarrow \mathbb{R}^{3} \supset S$ and inducing the $C^{\infty}$ complete tangent vector field $X:=f_{*} v$ on $S \subset \mathbb{R}^{3}$. As occurs with $v$, the flow $X^{t}$ has no minimal sets ( $f$ realizes a $C^{\infty}$ flow conjugation).

Definition. Let $\theta$ be a $C^{0}$ flow on a metric space $M$. A point $x \in M$ is called a limit point of $(M, \theta)$ if $x$ belongs to the $\alpha$-limit set or to the $\omega$-limit set of some point of $M$. In this case the orbit $\mathscr{O}(x)$ is called a limit orbit of the flow. We denote the set of limit points of the flow $(M, \theta)$ by $\Upsilon_{\theta}$ and if the flow is given by a vector field $v$, by $\Upsilon_{v}$.

From the inductively constructed tangentially orientable foliated atlas of $\mathfrak{M}$ corresponding to the vector field $v$ (given in [2]), it is easily seen that:

- each limit point $x \in \mathfrak{M}$ has nonvoid $\alpha$-limit and $\omega$-limit sets and both the positive and negative orbit of $x$ accumulate in the unique non-isolated end of $\mathfrak{M}$ and in no other end of this surface.

Since a homeomorphism between non-compact surfaces uniquely extends to a homeomorphism between their respective end-points compactifications, it follows that in the flow $X^{t}$ generated by $X \in \mathfrak{X}^{\infty}(S)$, both the $\alpha_{X}$-limit and $\omega_{X}$-limit sets of each limit point of $\left(S, X^{t}\right)$ are closed, unbounded ${ }^{46}$ subsets of $\mathbb{R}^{3}$. Let $U$ be a normal tubular neighbourhood of $S$ in $\mathbb{R}^{3}$ (indeed a trivial 1-dimensional vector bundle over $S)$. Extend $X:=f_{*} v \in \mathfrak{X}^{\infty}(S)$ to a non-singular vector field $X_{0} \in \mathfrak{X}^{\infty}(U)$ defining,

$$
\begin{aligned}
X_{0}: U & \longrightarrow \mathbb{R}^{3} \\
z & \longmapsto X \circ \pi(z)
\end{aligned}
$$

where $\pi: U \longrightarrow S$ is the canonical $C^{\infty}$ submersion (orthogonal projection of $U$ over $S){ }^{47}$ Let $p:=(0,0,0,1), O:=(0,0,0,0), \varphi: \mathbb{R}^{3} \longrightarrow \mathbb{S}^{3} \backslash\{p\}$ the

\footnotetext{
45 we need not to care with non-orientable ends since there are none: both $\mathfrak{M}$ and $S$ are orientable.

${ }^{46}$ both these sets are closed subsets of $S$ that do not accumulate in the isolated ends $e_{n}, n \in \mathbb{N}$ of this surface and the closure of $S$ in $\mathbb{R}^{3}$ equals $S \sqcup\left\{e_{n}: n \in \mathbb{N}\right\}$. Their unboundeness also follows from the fact that a closed, bounded subset of $\mathbb{R}^{3}$ is compact and thus if it is a nonvoid, invariant subset of the flow $X^{t}$, then it must contain a minimal set of it. But $X^{t}$ has no minimal sets.

${ }^{47}$ i.e. $U=\bigsqcup_{x \in S} \mathfrak{D}_{\lambda(x)}(x)$, where $\lambda \in C^{\infty}\left(S, \mathbb{R}^{+}\right), \mathfrak{D}_{\lambda(x)}(x)$ is the 1-dimensional affine open
} 
inverse stereographic projection $\left(\mathbb{R}^{3}\right.$ identified with $\left.\mathbb{R}^{3} \times\{-1\}\right)$. $\varphi$ induces the $C^{\infty}$ vector field $\varphi_{*} X_{0}$ on the open subset $\varphi(U)$ of $\mathbb{S}^{3}$.

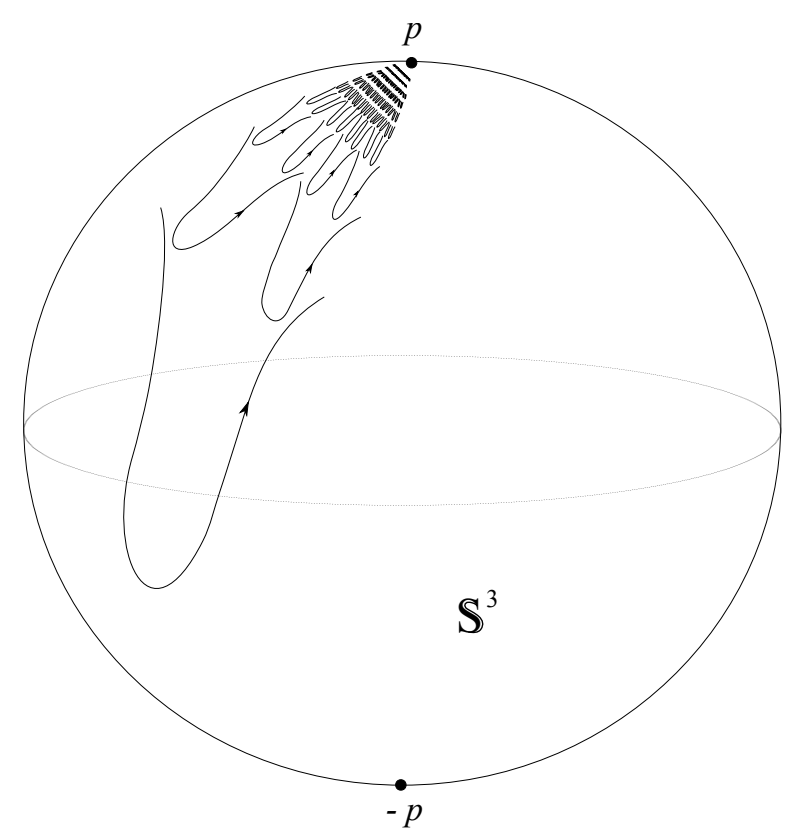

Fig. 17: $K$-tree in the flow $\left(\mathbb{S}^{3}, \zeta^{t}\right)$.

By Kaplan Smoothing Theorem (Kaplan [7], p.157), there is a scalar function $\lambda \in C^{\infty}\left(\mathbb{S}^{3},[0,1]\right)$ with $\lambda^{-1}(0)=\mathbb{S}^{3} \backslash \varphi(U)$ and such that

$$
\begin{aligned}
& \zeta: \mathbb{S}^{3} \subset \mathbb{R}^{4} \longrightarrow \mathbb{R}^{4} \\
& s \quad \longmapsto O \quad \text { on } \mathbb{S}^{3} \backslash \varphi(U) \\
& s \quad \longmapsto \lambda \varphi_{*} X_{0}(s) \quad \text { on } \varphi(U)
\end{aligned}
$$

defines a $C^{\infty}$ vector field on $\mathbb{S}^{3}$. The smoothly embedded surface $\varphi(S)$ is invariant under the flow $\zeta^{t}\left(\left.\varphi\right|_{S}\right.$ realizes a $C^{\infty}$ flow conjugation between the global flow $\left(S, X^{t}\right)$ and $\left(\varphi(S),\left(\varphi_{*} X\right)^{t}\right)$; moreover $\left.\left.\left.\operatorname{im} \lambda\right|_{\varphi(S)} \subset\right] 0,1\right]$, thus $\lambda \varphi_{*} X=\left.\zeta\right|_{\varphi(S)}$ is necessarily a complete vector field, topologically equivalent to $X \in \mathfrak{X}^{\infty}(S)$ via the smooth diffeomorphism $\varphi$ ).

Let $q \in \Upsilon_{X}$ and $z:=\varphi(q)$. Recall that $\alpha_{X}(q), \omega_{X}(q)$ and $\operatorname{cl} \mathscr{O}_{X}(q)$ are disk with centre $x \in S$ and radius $\lambda(x)$ orthogonal to $S$ at $x$ and $\pi: U \longrightarrow S$ is the $C^{\infty}$ submersion defined by:

$$
\pi\left(\mathfrak{D}_{\lambda(x)}(x)\right)=\{x\} \text { for all } x \in S
$$


unbounded, closed subsets of $\mathbb{R}^{3}$, therefore for all such $q$ and $z$,

$$
\begin{gathered}
\alpha_{\zeta}(z)=\varphi\left(\alpha_{X}(q)\right) \sqcup\{p\} \quad \text { and } \quad \omega_{\zeta}(z)=\varphi\left(\omega_{X}(q)\right) \sqcup\{p\} \\
\operatorname{cl} \mathscr{O}_{\zeta}(z)=\varphi\left(\operatorname{cl} \mathscr{O}_{X}(q)\right) \sqcup\{p\}
\end{gathered}
$$

Let $M:=\operatorname{cl} \mathscr{O}_{\zeta}(z), \quad \theta:=\left.\zeta^{t}\right|_{\mathbb{R} \times M}$ and $K:=\{p\} . \quad \theta$ is a $C^{0}$ flow on the compact, connected metric space $M \subset \mathbb{S}^{3}$ (with the euclidean metric of $\mathbb{R}^{4} \supset$ $\left.\mathbb{S}^{3}\right)$ and $K$ is a compact, invariant proper subset of $M$. Now with respect to the (sub)flow $(M, \theta)$, it is clear from (9) that every $y \in M \backslash K=M \backslash\{p\}$ belongs to $A_{\theta}^{-}(K) \cap A_{\theta}^{+}(K)$ as $M=\varphi\left(\operatorname{cl} \mathscr{O}_{X}(q)\right) \sqcup\{p\}$ and $\operatorname{cl} \mathscr{O}_{X}(q)=$ $\mathscr{O}_{X}(q) \cup \alpha_{X}(q) \cup \omega_{X}(q) \subset \Upsilon_{X}$. Obviously $K$ is isolated from minimals in $(M, \theta)$ and no $x \in M \backslash K$ has its $\alpha_{\theta}$-limit set or its $\omega_{\theta}$-limit set contained in $K$ (i.e. equal to $\{p\})$. This immediately implies that, for this choice of $M, \theta$ and $K$, none of conditions of Theorem 1, with the exception of condition 6, can hold. Therefore by Theorem 1 the above $M, \theta$ and $K$ necessarily provide a subsmooth independent realization of condition $\mathbf{6}$, with ambient manifold $\mathscr{M}=\mathbb{S}^{3}$. This can be easily verified directly: the existence of a $K$-tree, with $K=\{p\}$ and $\gamma_{0}=\mathscr{O}_{\theta}(z)$ is now a straightforward inductive consequence of Lemma 3 , since every $y \in M \backslash K$ belongs to $A_{\theta}^{-}(K) \cap A_{\theta}^{+}(K)$ and $K$ is consequently non-stagnant in $(M, \theta)$.

The example above provides the essential "dynamical piece" for the construction of a subsmooth independent realization of condition 7.6. But in order to do the same for conditions $\mathbf{8 . 6}$ and $\mathbf{9 . 6}$ we need to construct a subsmooth independent realization of condition $\mathbf{6}$ with $K$ a periodic orbit and one with $K$ a compact aperiodic minimal, respectively.

Example 2. subsmooth independent realization of condition $\mathbf{6}$ with $M$ an orbit closure of a $C^{\infty}$ flow $v^{t}$ on the ambient manifold $\mathscr{M}=\mathbb{S}^{3} \times \mathbb{S}^{1}, \theta=\left.v^{t}\right|_{\mathbb{R} \times M}$ and $K=\{p\} \times \mathbb{S}^{1} \subsetneq M$ a periodic orbit.

Endow the compact manifold $\mathscr{M}=\mathbb{S}^{3} \times \mathbb{S}^{1}$ with the $C^{\infty}$ vector field $v=$ $\zeta+\frac{\partial}{\partial \rho}$ where $\rho$ is the $\mathbb{S}^{1}$ coordinate. Call the circles $\{z\} \times \mathbb{S}^{1}, z \in \mathbb{S}^{3}$ the parallels of $\mathscr{M}$. The parallel $\gamma=\{p\} \times \mathbb{S}^{1}$ is a periodic orbit of $v$ (with period $2 \pi)$, since $p$ is a singularity of $\zeta \in \mathfrak{X}^{\infty}\left(\mathbb{S}^{3}\right)$. Observe that the flow $\left(\mathscr{M}, v^{t}\right)$ projects to the flow $\left(\mathbb{S}^{3}, \zeta^{t}\right)$ via the projection onto the 1 st factor $\pi_{1}$, more precisely, for every $t \in \mathbb{R}$ and $u \in \mathbb{S}^{3}, s \in \mathbb{S}^{1}$,

$$
\pi_{1} \circ v^{t}(u, s)=\zeta^{t}\left(\pi_{1}(u, s)\right)=\zeta^{t}(u)
$$




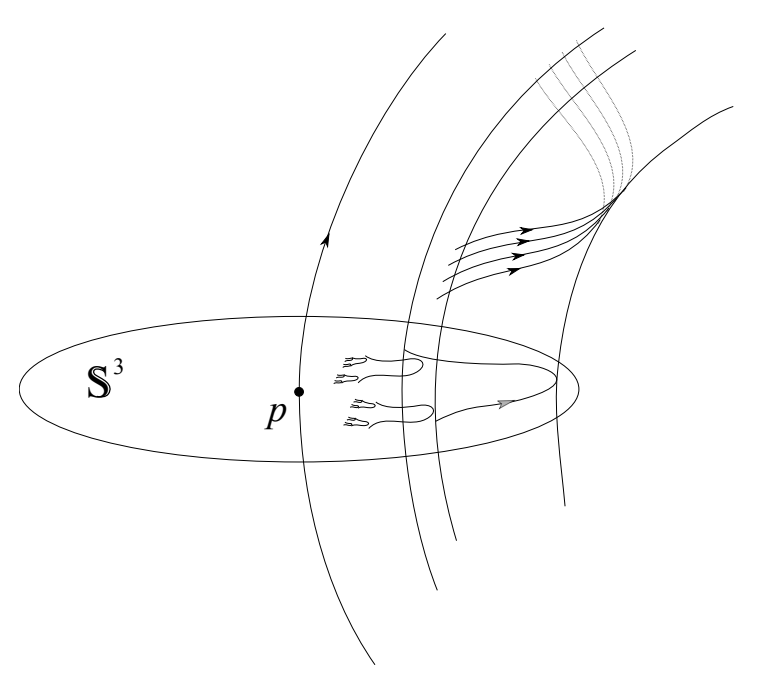

Fig. 18: Example 2, showing the projection ("shadow") of the flow $\left(\mathbb{S}^{3} \times \mathbb{S}^{1}, v^{t}\right)$ to the flow $\left(\mathbb{S}^{3}, \zeta^{t}\right)$.

and similarly $\left(\mathscr{M}, v^{t}\right)$ projects to the flow $\left(\mathbb{S}^{1},(\partial / \partial \rho)^{t}\right)$ via the projection onto the 2 nd factor $\pi_{2}$, and thus identifying $\mathbb{S}^{1}$ with $\mathbb{R} / 2 \pi \mathbb{Z}=\mathbb{R}(\bmod 2 \pi)$,

$$
v^{t}(u, s)=\left(\zeta^{t}(u), s+t(\bmod 2 \pi)\right)
$$

We will deduce all the facts needed about the dynamics of $v^{t}$ from these equalities. Observe that for every $u \in \mathbb{S}^{3}, s \in \mathbb{S}^{1}$,
a) $\pi_{1}\left(\mathscr{O}_{v}(u, s)\right)=\mathscr{O}_{\zeta}(u)$
b) $\pi_{1}\left(\omega_{v}(u, s)\right)=\omega_{\zeta}(u)$
c) $\pi_{1}\left(\alpha_{v}(u, s)\right)=\alpha_{\zeta}(u)$
d) $\pi_{1}\left(\operatorname{cl} \mathscr{O}_{v}(u, s)\right)=\operatorname{cl} \mathscr{O}_{\zeta}(u)$

a) is an immediate consequence of (10); b) and c) are time symmetric facts. To prove b) observe that the inclusion $\subset$ follows immediately from (10) and the reciprocal $\supset$ follows from the compactness of $\mathbb{S}^{1}$ : if $q \in \omega_{\zeta}(u)$, then for any $s \in \mathbb{S}^{1}=\mathbb{R}(\bmod 2 \pi), \omega_{v}(u, s)$ necessarily intercepts the parallel $\{q\} \times \mathbb{S}^{1}$. To see this, let $t_{n} \longrightarrow+\infty$ be such that $\zeta^{t_{n}}(u) \longrightarrow q \in \omega_{\zeta}(u)$. The sequence $s+t_{n}(\bmod 2 \pi)$ has a subsequence $\left(s+t_{n_{i}}(\bmod 2 \pi)\right)_{i \in \mathbb{N}}$ converging to some $s_{0}$ belonging to the compact $\mathbb{S}^{1}=\mathbb{R}(\bmod 2 \pi)$ and thus by (11),

$$
\lim _{i \rightarrow+\infty} v^{t_{n_{i}}}(u, s)=\lim _{i \rightarrow+\infty}\left(\zeta^{t_{n_{i}}}(z), s+t_{n_{i}}(\bmod 2 \pi)\right)=\left(q, s_{0}\right) \in \mathbb{S}^{3} \times \mathbb{S}^{1}
$$

therefore $\left(q, s_{0}\right) \in \omega_{v}(u, s)$ since $t_{n_{i}} \longrightarrow+\infty$. Finally, d) is an immediate consequence of $\mathrm{a}), \mathrm{b}$ ) and $\mathrm{c})$. 
Now let $z \in \varphi\left(\Upsilon_{X}\right) \in \mathbb{S}^{3}$ (see example 1) and $N:=\operatorname{cl} \mathscr{O}_{v}(z, b) \subset\left(\operatorname{cl}_{\zeta}(z)\right) \times \mathbb{S}^{1}$, $\phi:=\left.v^{t}\right|_{\mathbb{R} \times N}$ and $J:=\gamma=\{p\} \times \mathbb{S}^{1}$ (a periodic orbit, see above), where $b$ is an arbitrary point of $\mathbb{S}^{1}$. Observe that from the four identities above it follows that

$$
\begin{gathered}
(u, s) \in \operatorname{cl} \mathscr{O}_{v}(z, b) \backslash \gamma \Longrightarrow u \in \operatorname{cl} \mathscr{O}_{\zeta}(z) \backslash\{p\} \Longrightarrow \\
\Longrightarrow u \in A_{\zeta}^{-}(\{p\}) \cap A_{\zeta}^{+}(\{p\}) \Longrightarrow(u, s) \in A_{v}^{-}(J) \cap A_{v}^{+}(J) \Longrightarrow \\
\Longrightarrow(u, s) \in A_{\phi}^{-}(J) \cap A_{\phi}^{+}(J)
\end{gathered}
$$

Therefore $J$ is isolated from minimals in $(N, \phi)$ and no $x \in N \backslash J$ has its $\alpha_{\phi}$-limit set or its $\omega_{\phi}$-limit set contained in $J$ (i.e. equal to the periodic orbit $\left.\gamma=\{p\} \times \mathbb{S}^{1}\right)$. This immediately implies that, for this choice of $M:=N$, $\theta:=\phi$ and $K:=J$, none of conditions of Theorem 1, with the exception of condition 6, can hold. Therefore by Theorem 1 the above $N, \phi$ and $J$ necessarily provide a subsmooth independent realization of condition $\mathbf{6}$ with $J$ a periodic orbit, the ambient manifold being $\mathscr{M}=\mathbb{S}^{3} \times \mathbb{S}^{1}$.

Example 3. subsmooth independent realization of condition 6 with $M$ an orbit closure of a $C^{\infty}$ flow $v^{t}$ on the ambient manifold $\mathscr{N}=\mathbb{S}^{3} \times \mathbb{S}^{1} \times \mathbb{S}^{1}$, $\theta=\left.v^{t}\right|_{\mathbb{R} \times M}$ and $K=\{p\} \times \mathbb{S}^{1} \times \mathbb{S}^{1} \subsetneq M$ a compact aperiodic minimal.

Endow the compact manifold $\mathscr{N}=\mathbb{S}^{3} \times \mathbb{S}^{1} \times \mathbb{S}^{1}$ with the $C^{\infty}$ vector field $v=\zeta+\frac{\partial}{\partial \rho}+\sqrt{2} \frac{\partial}{\partial \sigma}=v+\sqrt{2} \frac{\partial}{\partial \sigma}$ where $\rho$ and $\sigma$ are, respectively, the coordinates of the left and of the right $\mathbb{S}^{1}$. Since $\{p\} \times \mathbb{S}^{1}$ is a periodic orbit of $v \in \mathfrak{X}^{\infty}\left(\mathbb{S}^{3} \times \mathbb{S}^{1}\right)$ with constant speed 1, the 2-torus $\{p\} \times \mathbb{S}^{1} \times \mathbb{S}^{1} \subset \mathscr{N}$ is invariant under $v^{t}$ and carries an irrational linear flow with slope $\sqrt{2}$. Again as in example 2 , the flow $\left(\mathbb{S}^{3} \times \mathbb{S}^{1} \times \mathbb{S}^{1}, v^{t}\right)$ projects to $\left(\mathbb{S}^{3} \times \mathbb{S}^{1}, v^{t}\right)$ via the projection $\pi_{1,2}$ onto the two first factors, and to $\left(\mathbb{S}^{1},(\sqrt{2} \partial / \partial \sigma)^{t}\right)$ via the projection $\pi_{3}$ onto the 3rd factor, hence for every $(u, s, w) \in \mathscr{N}$ and $t \in \mathbb{R}$,

$$
\begin{gathered}
\pi_{1,2} \circ v^{t}(u, s, w)=v^{t}\left(\pi_{1,2}(u, s, w)\right)=v^{t}(u, s) \\
v^{t}(u, s, w)=\left(v^{t}(u, s), \sqrt{2} t+w(\bmod 2 \pi)\right)
\end{gathered}
$$

By an argument entirely analogous to that employed in example 2, it follows that taking $z \in \varphi\left(\Upsilon_{X}\right) \in \mathbb{S}^{3}$ (see example 1), and letting $M:=\operatorname{cl} \mathscr{O}_{v}(z, b, c) \subset$ $\operatorname{cl} \mathscr{O}_{v}(z, b) \times \mathbb{S}^{1}, \quad \theta:=\left.v^{t}\right|_{\mathbb{R} \times M}$ and $K:=\{p\} \times \mathbb{S}^{1} \times \mathbb{S}^{1}$, where $b, c$ are arbitrary points of the first (left) and second (right) $\mathbb{S}^{1}$, respectively, we get a subsmooth independent realization of condition $\mathbf{6}$, with $K$ a compact aperiodic minimal (see above), the ambient manifold being $\mathscr{N}=\mathbb{S}^{3} \times \mathbb{S}^{1} \times \mathbb{S}^{1}$.

We will now briefly indicate how to obtain a subsmooth independent realization of condition 5 with $K$ an equilibrium orbit. For $K$ a periodic orbit or 
a compact aperiodic minimal we only have to proceed as in examples 2 and 3 . The analogue subsmooth independent realizations of condition $\mathbf{4}$ are obtained time-reversing the previously mentioned realizations of condition $\mathbf{5}$.

A subsmooth independent realization of condition 5 with $K$ an equilibrium orbit and ambient manifold $\mathbb{S}^{3}$ is achieved through a simple (and obvious) modification of Beniere and Meigniez's construction: in their paper ([2], p.23, bottom), the cut-and-paste operation that defines $M_{1}$ is performed only for each $p \in \mathbb{Z}_{0}^{+}$(instead of for all $p \in \mathbb{Z}$ ). Following otherwise their construction, we finally obtain a $C^{\infty}$ orientable surface $\mathfrak{N}$ of infinite genus, again with its end points set homeomorphic to $\Delta:=\{0\} \cup\{1 / n: n \in \mathbb{N}\} \subset \mathbb{R}$ and all ends flat except the non-isolated one. By Kerekjarto Theorem (see e.g. [10], p.262 and [2], p.26), this surface is thus $C^{\infty}$ diffeomorphic to the surface $\mathfrak{M}$ of example 1 (i.e. to the original surface carrying a flow without minimal set constructed in [2]) and hence to $S \subset \mathbb{R}^{3}$. N carries a $C^{\infty}$ vector field $v$ whose flow is no longer without minimal sets, as there are points $x \in \mathfrak{N}$ with $\alpha(x)=\emptyset=\omega(x)$. However for each limit point $x \in \mathfrak{N}$ of the flow $v^{t}$, it is easily seen that $\alpha(x)=\emptyset, \omega(x) \neq \emptyset$ and both the positive and negative orbits of $x$ accumulate in the unique non-isolated end of $\mathfrak{N}$ and in no other end of this surface. As in example 1 , we have again a $C^{\infty}$ diffeomorphism $f: \mathfrak{N} \longrightarrow S \subset \mathbb{R}^{3}$ defining a $C^{\infty}$ embedding $\mathfrak{N} \hookrightarrow \mathbb{R}^{3}$ and inducing a complete tangent vector field $X:=f_{*} v$ on $S \subset \mathbb{R}^{3}$. Then for each limit point $x$ of $\left(S, X^{t}\right), \omega_{X}(x)$ is a closed, unbounded subset of $\mathbb{R}^{3}$ and $\lim _{t \rightarrow-\infty}\left\|X^{t}(x)\right\|=+\infty \quad(\|\cdot\|$ being the euclidean norm on $\mathbb{R}^{3}$ ), i.e. the point $x$ "escapes" to infinite on $\mathbb{R}^{3}$ when $t \longrightarrow-\infty$. Then proceeding exactly as in example 1 , for each $q \in \Upsilon_{X}$, $z:=\varphi(q)$,

$$
\begin{gathered}
\alpha_{\zeta}(z)=\{p\} \quad \text { and } \quad \omega_{\zeta}(z)=\varphi\left(\omega_{X}(q)\right) \sqcup\{p\} \\
\operatorname{cl} \mathscr{O}_{\zeta}(z)=\varphi\left(\operatorname{cl} \mathscr{O}_{X}(q)\right) \sqcup\{p\}
\end{gathered}
$$

Letting $M:=\operatorname{cl} \mathscr{O}_{\zeta}(z) \subset \mathbb{S}^{3}, \quad \theta:=\left.\zeta^{t}\right|_{\mathbb{R} \times M}$ and $K:=\{p\}$, we then have

$$
y \in M \backslash K \Longrightarrow y \in B_{\theta}^{-}(K) \cap A_{\theta}^{+}(K)
$$

It is now immediate to verify that for these $M, \theta, K$, none of the 28 conditions of Theorem 1, with the exception of condition $\mathbf{5}$, can hold and therefore $(M, \theta)$, $K$ provide a subsmooth independent realization of condition 5 (see example 1).

We cannot enter here the details of the construction of subsmooth independent realization for the nine exceptional condition 7.4 to 9.6. In [12] such realizations are given for conditions $\mathbf{7 . 4}$ to $\mathbf{7 . 6}$ with ambient manifold $\mathbb{R}^{4}$, for $\mathbf{8 . 4}$ to 8.6 with ambient manifold $\mathbb{R}^{5}$ and for 9.4 to 9.6 with ambient manifold 
$\mathbb{R}^{6}$, the procedure being the same in all cases. The crucial fact is that $\mathbb{S}^{3}$, $\mathbb{S}^{3} \times \mathbb{S}^{1}, \mathbb{S}^{3} \times \mathbb{S}^{1} \times \mathbb{S}^{1}$ embed, respectively, as codimension one, closed $C^{\infty}$ submanifolds of $\mathbb{R}^{4}, \mathbb{R}^{5}$ and $\mathbb{R}^{6}$ (for the last two the open book decompositions of $\mathbb{R}^{5}$ and $\mathbb{R}^{6}$ are used), and consequently have trivial normal tubular neighbourhoods in the corresponding ambient manifolds.

\section{PROOF OF LEMMA 7. TWO TOPOLOGICAL LEMMAS.}

Lemma 7. Let $M$ be a locally compact metric space with a $C^{0}$ flow. If $A \subset M$ is open and $\operatorname{CMin}(D)$ is $d_{H}$-dense in itself then $\operatorname{CMin}(D)$ is $\mathfrak{c}$-dense in itself. If $\mathfrak{D}$ is a $d_{H}$-open and dense in itself subset of $\operatorname{CMin}(M)$ then $\mathfrak{D}$ is $\mathfrak{c}$-dense in itself.

Before entering the proof, a few technical definitions will be needed. Recall that $\mathrm{F}:=\{0,1\}$. For each $n \in \mathbb{N}, \mathrm{F}^{n}=\{0,1\}^{n}=$ the set of finite sequences of 0's and 1's with length $n$. Again, since no risk of ambiguity arises, commas and brackets are omitted in the representation of the elements of $\mathscr{F}$, thus we write, for example, 01 instead of $(0,1)$ and $\mathrm{F}^{1}$ is naturally identified with $\mathrm{F}$.

Let

$$
\mathrm{F}^{\leq n}:=\bigsqcup_{1 \leq m \leq n} \mathrm{~F}^{m} \quad \mathscr{F}:=\bigsqcup_{n \in \mathbb{N}} \mathrm{F}^{n} \quad \mathscr{F}_{\emptyset}:=\mathscr{F} \sqcup\{\emptyset\}
$$

( $\mathscr{F}_{\emptyset}$ is the set of finite sequences of 0's and 1's including the empty sequence $\emptyset)$. If $a, b \in \mathscr{F}_{\emptyset}, a b$ represents, as usual, the element of $\mathscr{F}_{\emptyset}$ obtained by adjoining $b$ to the right end of $a$ (naturally, $a \emptyset=a=\emptyset a$ for every $a \in \mathscr{F}_{\emptyset}$ ). For any $n \in \mathbb{N}$ and $a \in \mathrm{F}^{n},|a|:=n$ (the length of $a$ ). We now define the operators $*,+, \bullet,-$ on $\mathscr{F}$ :

$$
\begin{aligned}
& 0^{*}:=1, \quad 1^{*}:=0, \quad(b c)^{*}:=b c^{*} \\
& (b c)^{+}:=b c c^{*}, \quad(b c)^{\bullet}:=b c c \quad \text { for any } b \in \mathscr{F}_{\emptyset}, c \in \mathrm{F}, \\
& 0^{-}:=0, \quad 1^{-}:=0 \text { and }(b c)^{-}:=b \quad \text { for every } b \in \mathscr{F}, c \in \mathrm{F} .
\end{aligned}
$$

Obvious facts about $\mathscr{F}$ and its operators such as $\mathrm{F}^{n+1}=\left\{a c: a \in \mathrm{F}^{n}, c \in\right.$ $\mathrm{F}\}=\left(\mathrm{F}^{n}\right)^{+} \cup\left(\mathrm{F}^{n}\right)^{\bullet}$ will be implicitly used without mention.

Proof of Lemma 7. The first part of the lemma clearly implies the second: suppose $\mathfrak{D}$ is a $d_{H}$-open and dense in itself subset of $\operatorname{CMin}(M)$. By lemma 5 (section 5), for each $X \in \mathfrak{D}$, there is a $\delta_{X}>0$ such that $\operatorname{CMin}\left(B\left(X, \delta_{X}\right)\right) \subset \mathfrak{D}$ (as $\mathfrak{D}$ is $d_{H}$-open in $\mathrm{CMin}(M)$, there is an $\varepsilon_{X}>0$ such that $B_{H}\left(X, \varepsilon_{X}\right) \cap$ $\operatorname{CMin}(M) \subset \mathfrak{D}$. By lemma 5, there is a $\delta_{X}>0$ such that $\operatorname{CMin}\left(B\left(X, \delta_{X}\right)\right) \subset$ $\operatorname{Ci}\left(B\left(X, \delta_{X}\right)\right) \subset B_{H}\left(X, \varepsilon_{X}\right)$, hence $\left.\operatorname{CMin}\left(B\left(X, \delta_{X}\right)\right) \subset \mathfrak{D}\right)$. By lemma 8 , $\operatorname{CMin}\left(B\left(X, \delta_{X}\right)\right)$ is a $d_{H}$-open subset of $\operatorname{CMin}(M)$ and hence of $\mathfrak{D}$. $\mathfrak{D}$ 
is $d_{H}$-dense in itself (by hypothesis), and so is $\operatorname{CMin}\left(B\left(X, \delta_{X}\right)\right)$, since it is $d_{H}$-open in $\mathfrak{D}$. By the first part of lemma $7, \operatorname{CMin}\left(B\left(X, \delta_{X}\right)\right)$ is actually $\mathfrak{c}$-dense in itself, and obviously so is $\mathfrak{D}=\bigcup_{X \in \mathfrak{D}} \operatorname{CMin}\left(B\left(X, \delta_{X}\right)\right)$.

Proof of the 1st part of lemma 7 . Since $A$ is open and minimal sets are compact, given any $\Lambda_{0} \in \mathrm{CMin}(A), B\left(\Lambda_{0}, \varepsilon_{0}\right) \subset A$ for sufficiently small $\varepsilon_{0}>0$, hence by Lemma 6 (section 5) it is sufficient to prove that for any $\Lambda_{0} \in \operatorname{CMin}(A)$ and $\varepsilon_{0}>0$

$$
\# \operatorname{CMin}\left(B\left(\Lambda_{0}, \varepsilon_{0}\right)\right)=\mathfrak{c}
$$

The demonstration is based on a generalisation of the idea lying behind the construction of Cantor's ternary set: for each $\Lambda \in \operatorname{CMin}(A)$ and $\varepsilon>0$ such that $B[\Lambda, \varepsilon]$ is a compact subset of $A$, the existence of a continuum of $d_{H}$-Cauchy sequences of compact minimal sets $X \in \operatorname{CMin}(B(\Lambda, \varepsilon)) d_{H}-$ converging to a continuum of mutually disjoint, nonvoid, compact, connected invariant sets $Y \subset B(\Lambda, \varepsilon)$ is proved. The result then follows since each such $Y$ contains at least one compact minimal set of the flow.

Let $\Lambda_{0} \in \mathrm{CMin}(A)$ and $\varepsilon_{0}>0$ such that $B\left[\Lambda_{0}, \varepsilon_{0}\right] \subset A$ is compact. Since $\mathrm{CMin}(A)$ is $d_{H}$-dense in itself, there is a $\Lambda_{1} \in \mathrm{CMin}(A)$ such that

$$
\Lambda_{1} \in B_{H}\left(\Lambda_{0}, \varepsilon_{0}\right) \backslash\left\{\Lambda_{0}\right\}
$$

This implies $\Lambda_{1} \subset B\left(\Lambda_{0}, \varepsilon_{0}\right)$ and, $\left|\Lambda_{1}\right|_{\Lambda_{0}}<\varepsilon_{0}$ since $\Lambda_{1}$ is closed. Also $\Lambda_{0} \cap \Lambda_{1}=\emptyset$ since distinct minimal sets are disjoint. Let

$$
\varepsilon_{1}:=\min \left\{\operatorname{dist}\left(\Lambda_{0}, \Lambda_{1}\right), \varepsilon_{0}-\left|\Lambda_{1}\right|_{\Lambda_{0}}\right\} / 3
$$

Clearly, $\varepsilon_{1}>0, B\left[\Lambda_{0}, \varepsilon_{1}\right] \cap B\left[\Lambda_{1}, \varepsilon_{1}\right]=\emptyset$ and by the triangle inequality for the metric $d$ of $M$, it follows ${ }^{48}$ that $B\left[\Lambda_{1}, \varepsilon_{1}\right] \subset B\left(\Lambda_{0}, \varepsilon_{0}\right)$. Also $0<\varepsilon_{1}<\varepsilon_{0} / 3$ hence $B\left[\Lambda_{0}, \varepsilon_{1}\right] \subset B\left(\Lambda_{0}, \varepsilon_{0}\right)$.

Consider now the following proposition $\Theta(n)$ in the variable $n \in \mathbb{N}$ :

$\Lambda_{a} \in \mathrm{CMin}(A)$ and $\varepsilon_{m}>0$ are defined for all $a \in \mathrm{F}^{\leq n}$ and $1 \leq m \leq n$, respectively, and satisfy:

- for all $1 \leq m \leq n$,

a) $\varepsilon_{m}<\varepsilon_{m-1} / 3$

- for all $a \in \mathrm{F} \leq n$,

\footnotetext{
${ }^{48}$ by the same argument as that given in footnote [50] ahead, changing $n, b$ to 0 and $n+1$, $b^{+}$to 1 .
} 
b) $B\left[\Lambda_{a}, \varepsilon_{|a|}\right] \cap B\left[\Lambda_{a^{*}}, \varepsilon_{|a|}\right]=\emptyset$

c) $B\left[\Lambda_{a}, \varepsilon_{|a|}\right] \subset B\left(\Lambda_{a^{-}}, \varepsilon_{|a|-1}\right)$

Note that the truth of $\Theta(1)$ is already established. We prove that $\Theta(n) \Longrightarrow$ $\Theta(n+1)$. Assume $\Theta(n)$ is true. Observe that it is enough ${ }^{49}$ to define $\varepsilon_{n+1}>$ 0 and $\Lambda_{a} \in \operatorname{CMin}(A)$ for all $a \in \mathrm{F}^{n+1}$ and show that $\varepsilon_{n+1}<\varepsilon_{n} / 3$ and that

$$
B\left[\Lambda_{a}, \varepsilon_{n+1}\right] \cap B\left[\Lambda_{a^{*}}, \varepsilon_{n+1}\right]=\emptyset \text { and } B\left[\Lambda_{a}, \varepsilon_{n+1}\right] \subset B\left(\Lambda_{a^{-}}, \varepsilon_{n}\right)
$$

for all $a \in \mathrm{F}^{n+1}$. Define $\Lambda_{b^{\bullet}}:=\Lambda_{b}$ for every $b \in \mathrm{F}^{n}$. Again since $\operatorname{CMin}(A)$ is $d_{H}$-dense in itself, $\Lambda_{b} \in \mathrm{CMin}(A)$ and $\varepsilon_{n}>0$, we may select $\Lambda_{b^{+}} \in \operatorname{CMin}(A)$ satisfying

$$
\Lambda_{b^{+}} \in B_{H}\left(\Lambda_{b}, \varepsilon_{n}\right) \backslash\left\{\Lambda_{b}\right\}
$$

With each $a \in \mathrm{F}^{n+1}$ we have thus associated a compact minimal set contained in $A$. Now let $\varepsilon_{n+1}:=\min \left(\delta_{n+1}, \lambda_{n+1}\right)$ where

$$
\begin{aligned}
& \delta_{n+1}:=\min \left\{\operatorname{dist}\left(\Lambda_{b}, \Lambda_{b^{+}}\right): b \in \mathrm{F}^{n}\right\} / 3 \\
& \lambda_{n+1}:=\min \left\{\varepsilon_{n}-\left|\Lambda_{b^{+}}\right|_{\Lambda_{b}}: b \in \mathrm{F}^{n}\right\} / 3
\end{aligned}
$$

By (14), $\Lambda_{b}$ and $\Lambda_{b^{+}}$are distinct (compact) minimal sets hence disjoint, thus $\operatorname{dist}\left(\Lambda_{b}, \Lambda_{b^{+}}\right)>0 ; \Lambda_{b^{+}} \subset B\left(\Lambda_{b}, \varepsilon_{n}\right)$ and $\Lambda_{b^{+}}$is closed hence $\left|\Lambda_{b^{+}}\right|_{\Lambda_{b}}<\varepsilon_{n}$, therefore $\varepsilon_{n+1}>0$. Also, $\varepsilon_{n+1}<\varepsilon_{n} / 3$ since $\varepsilon_{n+1} \leq \lambda_{n+1}<\varepsilon_{n} / 3$. Observe that for any $a \in \mathrm{F}^{n+1}, \quad\left\{a, a^{*}\right\}=\left\{b^{\bullet}, b^{+}\right\}$where $b:=a^{-} \in \mathrm{F}^{n}$, thus $\left\{\Lambda_{a}, \Lambda_{a^{*}}\right\}=$ $\left\{\Lambda_{b}, \Lambda_{b^{+}}\right\}$since $\Lambda_{b^{\bullet}}=\Lambda_{b}$; therefore

$$
B\left[\Lambda_{a}, \varepsilon_{n+1}\right] \cap B\left[\Lambda_{a^{*}}, \varepsilon_{n+1}\right]=\emptyset
$$

since $\varepsilon_{n+1} \leq \delta_{n+1}$. To complete the induction it remains to show that

$$
B\left[\Lambda_{a}, \varepsilon_{n+1}\right] \subset B\left(\Lambda_{a^{-}}, \varepsilon_{n}\right) \text { for all } a \in \mathrm{F}^{n+1}
$$

Let $b:=a^{-}$; if $a=b^{\bullet}$ then $\Lambda_{a}=\Lambda_{b^{\bullet}}=\Lambda_{b}$ thus the inclusion is trivial since $\varepsilon_{n+1}<\varepsilon_{n} / 3<\varepsilon_{n}$; if $a=b^{+}$then $50\left[\Lambda_{b^{+}}, \varepsilon_{n+1}\right] \subset B\left(\Lambda_{b}, \varepsilon_{n}\right)$. The proof that $\Theta(n) \Longrightarrow \Theta(n+1)$ is complete. Therefore $\Lambda_{a} \in \mathrm{CMin}(A)$ and $\varepsilon_{n}>0$ are

\footnotetext{
${ }^{49}$ since all the other conditions are guaranteed to be true by the hypothesis that $\Theta(n)$ is true.

${ }^{50}$ by the triangle inequality for the the metric $d$ of $M, \Lambda_{b^{+}} \subset B\left[\Lambda_{b},\left|\Lambda_{b^{+}}\right|_{\Lambda_{b}}\right]$ hence $B\left[\Lambda_{b^{+}}, \varepsilon_{n+1}\right] \subset B\left[\Lambda_{b},\left|\Lambda_{b^{+}}\right|_{\Lambda_{b}}+\varepsilon_{n+1}\right]$ and since $\left|\Lambda_{b^{+}}\right|_{\Lambda_{b}}<\varepsilon_{n}$, it follows that $\left|\Lambda_{b^{+}}\right|_{\Lambda_{b}}+$ $\varepsilon_{n+1} \leq\left|\Lambda_{b^{+}}\right|_{\Lambda_{b}}+\left(\varepsilon_{n}-\left|\Lambda_{b^{+}}\right|_{\Lambda_{b}}\right) / 3<\varepsilon_{n}$, thus $B\left[\Lambda_{b^{+}}, \varepsilon_{n+1}\right] \subset B\left[\Lambda_{b},\left|\Lambda_{b^{+}}\right|_{\Lambda_{b}}+\varepsilon_{n+1}\right] \subset$ $B\left(\Lambda_{b}, \varepsilon_{n}\right)$.
} 
defined for all $a \in \mathscr{F}$ and $n \in \mathbb{N}$, respectively and are such that condition a) of $\Theta(n)$ is true for all $m \in \mathbb{N}$ and conditions b) and c) are true for all $a \in \mathscr{F}$. Using induction again, it is now simple to deduce from c) that

$$
\begin{gathered}
B\left[\Lambda_{a}, \varepsilon_{|a|}\right] \subset B\left(\Lambda_{0}, \varepsilon_{0}\right) \text { for all } a \in \mathscr{F} \\
B\left[\Lambda_{a b}, \varepsilon_{|a b|}\right] \subset B\left(\Lambda_{a}, \varepsilon_{|a|}\right) \text { for all } a, b \in \mathscr{F}
\end{gathered}
$$

To conclude the proof we associate with each infinite sequence of 0 's and 1 's, $w \in \mathrm{F}^{\mathbb{N}}$, a compact minimal set $\Gamma_{w} \in \operatorname{CMin}\left(B\left(\Lambda_{0}, \varepsilon_{0}\right)\right)$ and show that for all $w, v \in \mathrm{F}^{\mathbb{N}}, \quad w \neq v$ implies $\Gamma_{w} \neq \Gamma_{v}$, therefore proving the existence of a continuum of compact minimal sets contained in $B\left(\Lambda_{0}, \varepsilon_{0}\right)$, since $\# \mathrm{~F}^{\mathbb{N}}=\mathfrak{c}$. For each $w \in \mathrm{F}^{\mathbb{N}}$ let $w_{n} \in \mathrm{F}^{n}$ denote the sequence of the first $n$ digits of $w$. We claim that $\left(\Lambda_{w_{n}}\right)$ is a $d_{H}$-Cauchy sequence in the compact metric space $\mathfrak{S}\left(B\left[\Lambda_{0}, \varepsilon_{0}\right]\right)$. Observe that together, the triangle inequality for the $d_{H}$ metric of $\mathrm{C}(M), \Lambda_{b c} \in B_{H}\left(\Lambda_{b}, \varepsilon_{|b|}\right)$ for all $b \in \mathscr{F}, c \in \mathrm{F}$ and $\varepsilon_{n+1}<\varepsilon_{n} / 3$ imply that for all $n, k \in \mathbb{N}$,

$$
\Lambda_{w_{n+k}} \in B_{H}\left(\Lambda_{w_{n}}, \varepsilon_{n} \cdot\left(\sum_{j=0}^{k-1} \frac{1}{3^{j}}\right)\right)
$$

and since $\varepsilon_{n}<\varepsilon_{0} / 3^{n}$ it follows that for any $p, q>n$

$$
\Lambda_{w_{p}}, \Lambda_{w_{q}} \in B_{H}\left(\Lambda_{w_{n}}, \frac{\varepsilon_{0}}{2 \cdot 3^{n-1}}\right)
$$

hence

$$
d_{H}\left(\Lambda_{w_{p}}, \Lambda_{w_{q}}\right)<2 \cdot \frac{\varepsilon_{0}}{2 \cdot 3^{n-1}}=\frac{\varepsilon_{0}}{3^{n-1}}
$$

therefore the sequence $\left(\Lambda_{w_{n}}\right)$ is clearly $d_{H}$-Cauchy and thus $d_{H}$-convergent. Since for all $n \in \mathbb{N}$,

$$
\Lambda_{w_{n}} \subset B\left[\Lambda_{w_{1}}, \varepsilon_{1}\right] \subset B\left(\Lambda_{0}, \varepsilon_{0}\right)
$$

it follows that

$$
\lim \Lambda_{w_{n}}=: \Lambda_{w} \in \mathfrak{S}\left(B\left(\Lambda_{0}, \varepsilon_{0}\right)\right)
$$

On the other hand, if $v, w \in \mathrm{F}^{\mathbb{N}}$ and $v \neq w$ then there is a $m \in \mathbb{N}$ such that $v_{m}=w_{m}^{*}$, thus

$$
\begin{gathered}
\left(\Lambda_{w_{n}} \in B\left(\Lambda_{w_{m}}, \varepsilon_{m}\right) \text { for any } n>m\right) \Longrightarrow \Lambda_{w} \subset B\left[\Lambda_{w_{m}}, \varepsilon_{m}\right] \\
\left(\Lambda_{v_{n}} \in B\left(\Lambda_{v_{m}}, \varepsilon_{m}\right) \text { for any } n>m\right) \Longrightarrow \Lambda_{v} \subset B\left[\Lambda_{v_{m}}, \varepsilon_{m}\right]=B\left[\Lambda_{w_{m}^{*}}, \varepsilon_{m}\right]
\end{gathered}
$$


therefore $\Lambda_{w} \cap \Lambda_{v}=\emptyset$ since $B\left[\Lambda_{w_{m}}, \varepsilon_{m}\right] \cap B\left[\Lambda_{w_{m}^{*}}, \varepsilon_{m}\right]=\emptyset$. This establishes the existence of a continuum of mutually disjoint, nonvoid, compact, connected, invariant sets $\Lambda_{w} \in \mathfrak{S}\left(B\left(\Lambda_{0}, \varepsilon_{0}\right)\right), w \in \mathrm{F}^{\mathbb{N}}$ and each such $\Lambda_{w}$ contains necessarily at least one compact minimal set of the flow $\Gamma_{w} \in \operatorname{CMin}\left(B\left(\Lambda_{0}, \varepsilon_{0}\right)\right) \subset$ $\operatorname{CMin}(A) \cdot 51$

8.2 Recall that every locally compact, connected mertic space is separable.

Proposition. If $M$ is a locally compact, separable metric space then so is $\left[\mathrm{C}(M), d_{H}\right]$, where $\mathrm{C}(M)$ is the set of all nonvoid compact subsets of $M$.

Proof. Claim 1: $\left[\mathrm{C}(M), d_{H}\right]$ is locally compact. Since $M$ is locally compact, given any $K \in \mathrm{C}(M)$ there is an $\varepsilon>0$ such that $B[K, \varepsilon]$ is compact. By Blaschske Theorem (see section 2) $\mathrm{C}(B[K, \varepsilon])$ is $d_{H}$-compact. Since $X \in B_{H}(K, \varepsilon) \Longrightarrow X \subset B(K, \varepsilon) \Longrightarrow X \in \mathrm{C}(B[K, \varepsilon]), \mathrm{C}(B[K, \varepsilon])$ is a $d_{H}$-compact neighbourhood of $K$ in $\left[\mathrm{C}(M), d_{H}\right]$, thus this last metric space is locally compact.

Claim 2: $\left[\mathrm{C}(M), d_{H}\right]$ is separable. Since $M$ is locally compact and separable, it admits an equivalent boundedly compact metric $d^{\prime}$ (every closed and bounded subset is compact, see e.g. [9], p.278). Fixing $O \in M, M=$ $\bigcup_{n \geq 1} B_{d^{\prime}}[O, n]$ defines an exhaustion of $M$ by compact subsets. Again by Blaschke Theorem, $C\left(B_{d^{\prime}}[O, n]\right)$ is $d_{H}^{\prime}$-compact for every $n \geq 1$ and hence $d_{H}^{\prime}$-separable, thus $\left[\mathrm{C}(M), d_{H}^{\prime}\right]$ is separable since $\mathrm{C}(M)=\bigcup_{n \geq 1} \mathrm{C}\left(B_{d^{\prime}}[O, n]\right)$. Now $d_{H}$ and $d_{H}^{\prime}$ are equivalent metrics on $\mathrm{C}(M)$ since $d$ and $d^{\prime}$ are equivalent on $M$, hence $\left[\mathrm{C}(M), d_{H}\right]$ is separable.

\subsection{Proof of $\mathrm{CH} \Longleftrightarrow \mathfrak{c D H}$ :}

$(\mathrm{CH} \Longrightarrow \mathfrak{c D H})$ : recall that a separable metric space $L$ has at most a continuum of points. Suppose $L$ is uncountable. Let $\mathfrak{I}$ be the set of points of $L$ for which there is an $\varepsilon>0$ such that $B(x, \varepsilon)$ is countable. For each $x \in \mathfrak{I}$ define

$$
\varepsilon_{x}:=\sup \{\varepsilon>0: B(x, \varepsilon) \text { is countable }\}
$$

Now observe that in the proof of Theorem 3, from the beginning until the end of claim 1 we have only used the fact that $\left[\operatorname{CMin}(M), d_{H}\right]$ is an uncountable

\footnotetext{
${ }^{51}$ Remark: Observe that even assuming the Continuum Hypothesis, a standard Baire category argument is not enough, without further assumptions, to prove Lemma 7. Suppose that $\Lambda_{0} \in \mathrm{CMin}(A)$ and $\varepsilon_{0}>0$ are such that $B\left[\Lambda_{0}, \varepsilon_{0}\right]$ is a compact subset of $A$. Since CMin $(A)$ is $d_{H}$-dense in itself, $\mathfrak{I}:=\mathrm{cl}_{H} \mathrm{CMin}\left(B\left(\Lambda_{0}, \varepsilon_{0}\right)\right)$ is easily seen to be a $d_{H}$-dense in itself and compact subset of $\mathfrak{S}\left(B\left[\Lambda_{0}, \varepsilon_{0}\right]\right)$. By Baire Theorem, if $\mathfrak{I}$ is nonvoid then $\mathfrak{I}$ cannot be countable, therefore assuming the Continuum Hypothesis, \# $\mathfrak{I} \geq \mathfrak{c}$, i.e. $\mathfrak{I}$ contains a continuum of distinct compact, connected, invariant subsets of $B\left[\Lambda_{0}, \varepsilon_{0}\right]$. However a priori nothing guarantees that these sets are mutually disjoint, thus the possibility of associating with each $X \in \mathfrak{I}$ a distinct (and hence disjoint) compact minimal set contained in $X$ is compromised.
} 
separable metric, hence all facts proved until there are valid for arbitrary uncountable separable metric spaces. In particular $\mathfrak{I}$ is a countable, open subset of $L$ and $\mathfrak{D}:=L \backslash \mathfrak{I}$ is a set such that every neighbourhood $U_{z}$ of each $z \in \mathfrak{D}$ contains uncountably many points of $\mathfrak{D}$. As the cardinal of $L \supset \mathfrak{D}$ is at most $\mathfrak{c}=2^{\aleph_{0}}$, the Continuum Hypothesis actually implies \# $\left(U_{z} \cap \mathfrak{D}\right)=\mathfrak{c}$. Hence $\mathfrak{D}$ is $\mathfrak{c}$-dense in itself.

$(\neg \mathrm{CH} \Longrightarrow \neg \mathfrak{c} D H):$ assume there is a cardinal $\boldsymbol{\aleph}_{0}<\beta<\mathfrak{c}$. Given the bijection between the cardinal $\mathfrak{c} \supset \beta$ and $\mathbb{R}$, there is a set $S \subset \mathbb{R}$ with $\mathfrak{\aleph}_{0}<\# S=\beta<\mathfrak{c}$. With the euclidean metric inherited from $\mathbb{R}, S$ is an uncountable separable metric space. Removing from $S$ an arbitrary countable set $\mathfrak{I}$ we obtain again a set $\mathfrak{D}=S \backslash \mathfrak{I}$ of cardinal $\# S=\#(S \backslash \mathfrak{I})=\beta$. Hence $\mathfrak{\aleph}_{0}<\# \mathfrak{D}=\beta<\mathfrak{c}$ and therefore, since $\mathfrak{D}$ is nonvoid, it cannot be $\mathfrak{c}$-dense in itself.

Acknowledgement: the author wishes to thank Jorge Rocha (FCUP - Portugal) and Vítor Araújo (UFB - Brasil), for their helpful comments and suggestions, leading to an improvement of the presentation.

\section{References}

[1] N. Bhatia, G. Szegö. Stability theory of dynamical systems. SpringerVerlag, Berlin 1970.

[2] J.-C. Beniere and G. Meigniez. Flows without minimal set. Ergodic Th. and Dynam. Sys. (1999), 19, 21-30.

[3] D. Burago, Y. Burago, S. Ivanov. A course in metric geometry. Graduate studies in mathematics vol. 33 - AMS, Providence, Rhode Island 2001.

[4] G. Butler, P. Waltman. Persistence in dynamical systems. Journ. of Diff. Eq. (1986), 63, No. 2, 255-263.

[5] C. Gutierrez. Smoothing continuous flows on two-manifolds and recurrences. Ergodic Th. and Dynam. Sys. (1986), 6, 17-44.

[6] T. Inaba. An example of a flow on a non-compact surface without minimal set. Ergodic Th. and Dynam. Sys. (1999), 19, 31-33.

[7] W. Kaplan. The singularities of continuous functions and vector fields. Michigan Math. J., 9, issue 2 (1962), 151-159.

[8] A. Levy. Basic set theory. Springer-Verlag, Berlin-Heidelberg 1979. 
[9] E. Lima. Espaços métricos. IMPA - Projecto Euclides (1983).

[10] I. Richards. On the classification of noncompact surfaces. Trans. Amer. Math. Soc. 106 (1963), 259-269.

[11] H. Smith, H. Thieme. Dynamical systems and population persistence. Graduate studies in mathematics vol. 118 - AMS, Providence, Rhode Island (2011)

[12] P. Teixeira. Flows near compact invariant sets - examples. arXiv (to appear).

[13] T. Ura, I. Kimura. Sur le courant extérieur à une region invariante: Théorème de Bendixson. Comm. Math Univ. St. Paul 8, 23-39 (1960).

E-mail address: pedro.teixeira@fc.up.pt

Centro de Matemática da Universidade do Porto

Rua do Campo Alegre, 687

4169-007 Porto

Portugal 